\title{
Dynamic responses of the adrenal steroidogenic regulatory network
}

\author{
Francesca Spiga ${ }^{a, 1,2}$, Eder Zavala ${ }^{b, c, d, 1}$, Jamie J. Walkera,c,d,e,1, Zidong Zhao ${ }^{a}$, John R. Terry ${ }^{b, c, d}$, \\ and Stafford L. Lightman ${ }^{\text {a,d }}$
}

\begin{abstract}
${ }^{a}$ Henry Wellcome Laboratories for Integrative Neuroscience and Endocrinology, University of Bristol, Bristol BS1 3NY, United Kingdom; ${ }^{b}$ Living Systems Institute, University of Exeter, Exeter EX4 4QD, United Kingdom; 'Wellcome Trust Centre for Biomedical Modelling and Analysis, University of Exeter, Exeter EX4 4QD, United Kingdom; ${ }^{d}$ EPSRC Centre for Predictive Modelling in Healthcare, University of Exeter, Exeter EX4 4QD, United Kingdom;

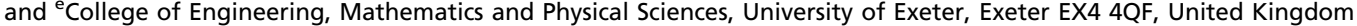

Edited by Bruce S. McEwen, The Rockefeller University, New York, NY, and approved June 22, 2017 (received for review March 8, 2017)

\begin{abstract}
The hypothalamic-pituitary-adrenal axis is a dynamic system regulating glucocorticoid hormone synthesis in the adrenal glands. Many key factors within the adrenal steroidogenic pathway have been identified and studied, but little is known about how these factors function collectively as a dynamic network of interacting components. To investigate this, we developed a mathematical model of the adrenal steroidogenic regulatory network that accounts for key regulatory processes occurring at different timescales. We used our model to predict the time evolution of steroidogenesis in response to physiological adrenocorticotropic hormone (ACTH) perturbations, ranging from basal pulses to larger stress-like stimulations (e.g., inflammatory stress). Testing these predictions experimentally in the rat, our results show that the steroidogenic regulatory network architecture is sufficient to respond to both small and large ACTH perturbations, but coupling this regulatory network with the immune pathway is necessary to explain the dissociated dynamics between ACTH and glucocorticoids observed under conditions of inflammatory stress.
\end{abstract}

adrenal gland | glucocorticoids | steroidogenesis | stress response | mathematical modeling

$T^{\mathrm{h}}$ he hypothalamic-pituitary-adrenal (HPA) axis is a stressresponsive neuroendocrine system that controls circulating levels of the vital glucocorticoid (CORT) hormones corticosterone (in rodents) and cortisol (in humans). These are steroids synthesized by the adrenal gland in response to stimulation by adrenocorticotropic hormone (ACTH), which is secreted by the anterior pituitary in response to corticotrophin-releasing hormone (CRH) and arginine vasopressin released from hypothalamic paraventricular neurons. These neurons receive circadian inputs from the suprachiasmatic nucleus and are activated in response to stress. Via the bloodstream, CORT accesses target tissues where it mediates metabolic, cognitive, and immune responses. CORT also regulates its own production through negative feedback inhibition of ACTH and $\mathrm{CRH}$ secretion from the pituitary and hypothalamus, respectively. To mount an effective response to stress, CORT must be secreted rapidly by the adrenal glands. However, because of its lipophilic nature, CORT cannot be prestored in vesicles and must therefore be rapidly synthesized de novo in response to ACTH stimulation.

Under basal, unstressed conditions, ACTH and CORT exhibit ultradian oscillations. Although there is some evidence for pulsatility of CRH $(1,2)$, our recent work suggests that ACTH and CORT pulsatility is predominantly generated by a subhypothalamic oscillator within the pituitary-adrenal system $(3,4)$. The amplitude of these pulses varies in a circadian manner with larger pulses occurring at the start of the active phase (morning in humans, evening in rodents). Under normal physiological conditions, CORT secretion is tightly correlated with ACTH (5). However, there are a number of conditions where a dynamic dissociation between these hormones occurs (reviewed in ref. 6). For example, there is evidence that proinflammatory cytokines released during inflammation can potentiate adrenal responsiveness to $\mathrm{ACTH}$ and can activate the adrenal steroidogenic pathway directly (7). Recent examples of this are the hormonal stress responses observed during cardiac surgery in humans and in a rodent model of inflammation (8). It has been hypothesized that this dynamic dissociation is due to increased adrenal sensitivity to ACTH, presumably as a result of the effect of circulating proinflammatory cytokines. Thus, a better understanding of the mechanisms that regulate steroidogenesis is necessary to explain the dynamic response of CORT to both physiological and pathological ACTH perturbations.

Within cells of the adrenal cortex zona fasciculata, steroidogenesis is regulated by several processes operating over a range of timescales: there are rapid nongenomic processes, as well as slower processes that depend on gene expression. Together, these form a complex pathway that is activated when ACTH binds the melanocortin type-2 receptor (MC2R), resulting in rapid phosphorylation of proteins involved in cholesterol metabolism-the substrate for CORT synthesis. These include hormone-sensitive lipase (HSL) and steroidogenic acute regulatory (StAR) proteins, which control levels of intracellular cholesterol and its transport within the mitochondrial matrix, respectively $(9,10)$. In addition to these rapid, nongenomic events, ACTH simultaneously triggers a slower genomic response involving the expression of steroidogenic genes such as StAR, CYP11A, MC2R, and MRAP. These genes are transcriptionally regulated by a number of nuclear receptors, the activity and expression of which are also under the control of ACTH. Specifically, ACTH induces the "positive regulators" steroidogenic factor 1 (SF-1) (11) and Nur77 (NR4A1) (12) and inhibits the "negative regulator" DAX-1 (dosage sensitive sex-reversal, adrenal hypoplasia

\section{Significance}

Our ability to respond to stress depends on a remarkably dynamic process of hormone secretion. The rapid release of glucocorticoid hormones from the adrenal glands is critical to mount such an efficient response to stress, particularly during inflammation. Although many key factors involved in this process have been studied, the way in which these factors interact dynamically with one another to regulate glucocorticoid secretion has not been investigated. Here, we develop a mathematical model of the regulatory network controlling glucocorticoid synthesis and, by combining this with in vivo experiments, show how this network governs changes in adrenal responsiveness under basal unstressed physiological conditions and under exposure to inflammatory stress.

Author contributions: F.S., J.J.W., J.R.T., and S.L.L. designed research; F.S. and Z.Z. performed experiments; F.S., E.Z., and J.J.W. analyzed data; E.Z. and J.J.W. developed and analyzed mathematical models; F.S., E.Z., and J.J.W. wrote the paper with revision by all authors.

The authors declare no conflict of interest.

This article is a PNAS Direct Submission.

Freely available online through the PNAS open access option.

${ }^{1}$ F.S., E.Z., and J.J.W. contributed equally to this work.

${ }^{2}$ To whom correspondence should be addressed. Email: f.spiga@bristol.ac.uk.

This article contains supporting information online at www.pnas.org/lookup/suppl/doi:10. 1073/pnas.1703779114/-/DCSupplemental. 
congenita locus on the $\mathrm{X}$ chromosome) (13). These interacting cascades of genomic and nongenomic events work in combination to activate and maintain optimal levels of steroidogenic proteins, ultimately leading to mitochondrial import of cholesterol and CORT synthesis. In addition, our recent mathematical modeling of the CORT response to ACTH suggests that a rapid, intra-adrenal CORT negative feedback loop constitutes an additional control mechanism of steroidogenesis (14-17). The biological processes underlying this self-inhibition are not known, but the glucocorticoid receptor $(\mathrm{GR})$ is expressed in the adrenal cortex $(15,18)$. Furthermore, the synthetic glucocorticoid dexamethasone-a specific GR agonist-has been shown to inhibit ACTH-induced corticosterone secretion from rat adrenocortical cells (19) as well as the transcription of steroidogenic genes through a mechanism that involves GRand SF-1-mediated induction of DAX-1 expression (20).

Although many of the components involved in the steroidogenic pathway have been identified, it remains unclear how their mutual interaction regulates CORT dynamics. In this study, we considered the core components of the steroidogenic regulatory network (SRN) and investigated how the cross-talk among these components underlies ACTH-responsive CORT dynamics. To understand the time evolution of steroidogenesis following ACTH stimulation, we developed a mathematical model of the SRN based on the complex molecular interactions within the SRN, including the intraadrenal GR-CORT connection that feeds back on the genomic pathway. We used this model to characterize dynamic responses to a variety of perturbations and to make predictions that we then tested experimentally. Our results show that it is the complex interactions between components of the SRN that govern dynamic steroidogenic responses in adrenocortical cells. Importantly, the SRN architecture enables cells to respond to both small and large ACTH perturbations, but is not sufficient to explain the dynamic response to inflammatory stress; in this case, we found that the CORT response can only be explained by coupling the SRN with the immune pathway. This work provides a theoretical framework to study adrenal CORT responses to a range of physiological and pathological perturbations, building upon our previous work on the role of glucocorticoid-mediated negative feedback loops regulating the ultradian dynamics of the HPA axis $(3,4)$.

\section{Results}

A Short Pulse of ACTH Dynamically Activates the SRN. To explore systematically how dynamic responses result from complex interactions within the SRN, we developed a mathematical model of the regulatory network. To aid analysis, we restrained the complexity of the model by stripping off nonessential regulatory components from the network architecture. This was performed by including only those nodes within the SRN that have been shown to be involved in CORT-mediated feedback loops and participate in cross-talk with StAR (Fig. 1; see SI Appendix, Fig. S1 for the full SRN) (20-25). Specifically, the model consists of a set of delay differential equations (DDEs) that describe the dynamics of intra-adrenal levels of CORT (A-CORT) and phosphorylation of GR (pGR, a marker of GR activation), and the expression of DAX-1, SF-1 and StAR following "virtual" (in silico) stimulation by ACTH (SI Appendix, Fig. S2 and Mathematical Model).

To assist the calibration of our mathematical model, we first considered the response of the SRN to a single pulse of ACTH, which is similar to an endogenous ultradian pulse of ACTH (Fig. $2 A$ ). To do this, we performed an in vivo experiment in the rat to characterize the dynamic response of the adrenal SRN to an i.v. injection of ACTH. We measured steroidogenic factors in the core SRN (Fig. 1), as well as some additional factors that are also involved in steroidogenesis but were not considered in the model. The i.v. ACTH injection resulted in a rapid and transient increase in plasma $\mathrm{ACTH}$ (Fig. $2 A$ ) with a peak concentration comparable to the peak of endogenous ultradian ACTH pulses $(26,27)$. This was accompanied by a rapid and dynamic increase in CORT secretion, detectable in both adrenal tissue (A-CORT; Fig. 2B) and blood plasma (P-CORT; SI Appendix, Fig. S3B). To assess the dynamics of GR activation following the i.v. ACTH injection, we measured pGR at Serine 211 (28). pGR demonstrated a rapid, significant increase (Fig. 2C), and its pattern of activation was similar to the observed pattern of A-CORT (Fig. $2 B$ ).

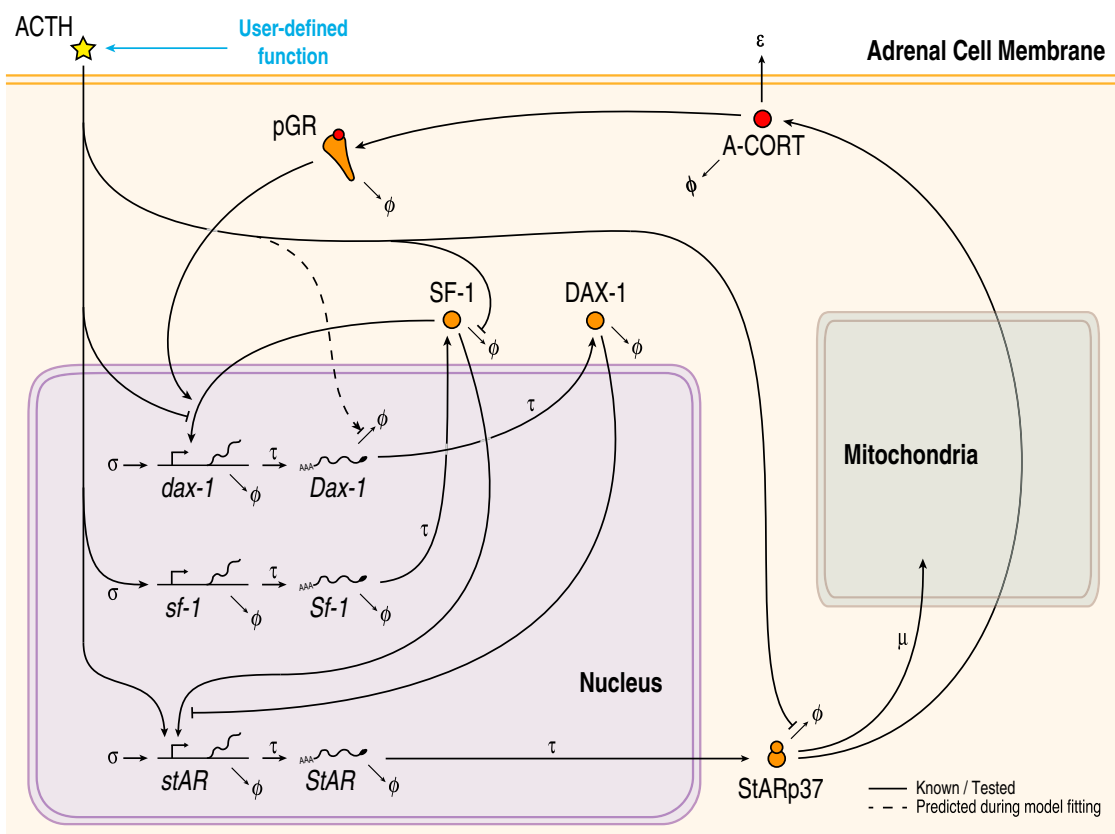

Fig. 1. The simplified SRN represented in the mathematical model accounts for both genomic and nongenomic processes occurring at different time scales. Also considered in the model are the A-CORT/GR-mediated intra-adrenal feedback loop and posttranscriptional and posttranslational processes, including the ACTH-mediated stabilization of StARp37. 

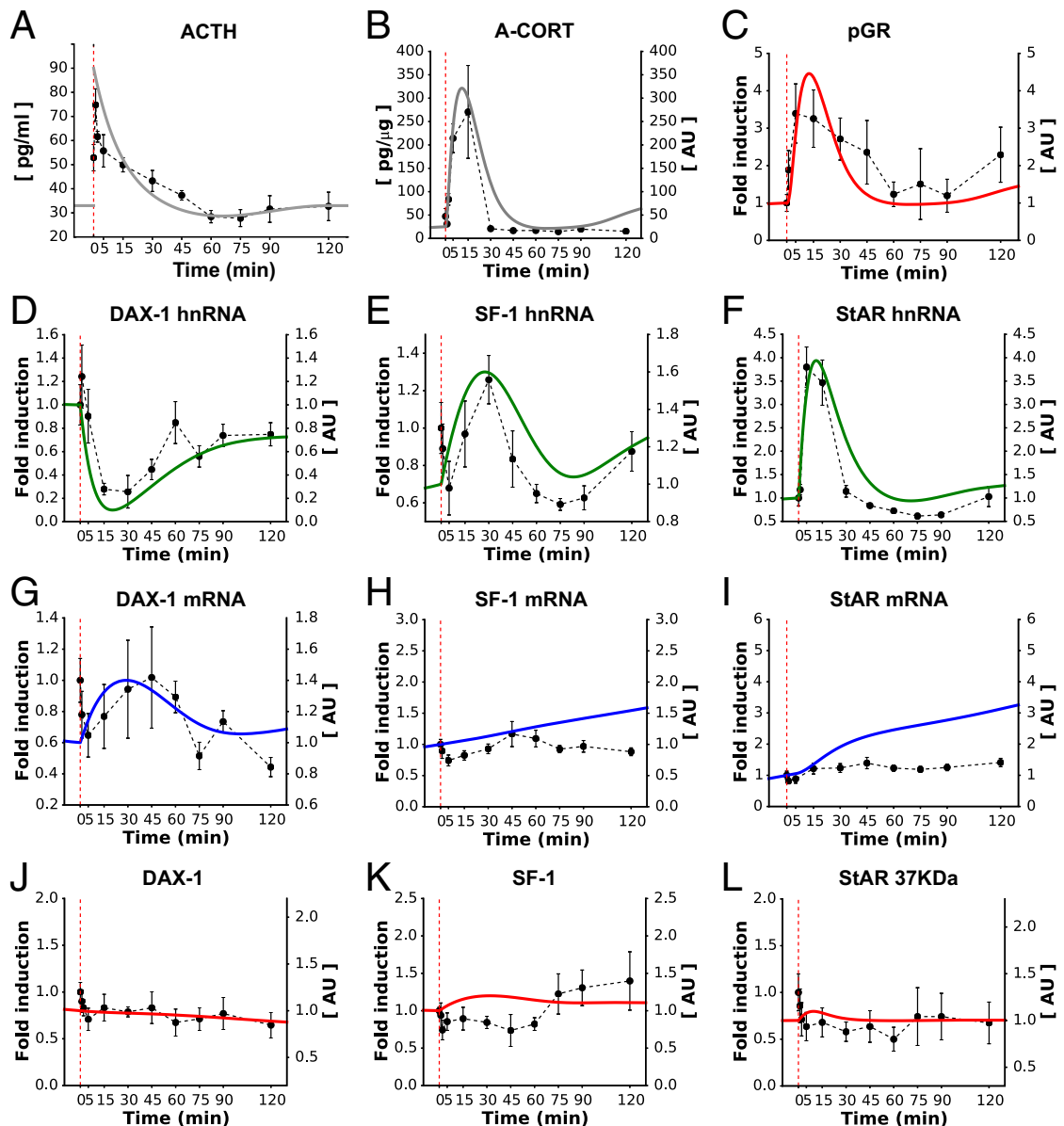

Fig. 2. The calibrated model reproduced the SRN dynamics following a short pulse of ACTH (A). Fitting the model to data successfully reproduced the rapid and transient synthesis of A-CORT $(B)$ and GR activation (C). The model also made qualitative predictions about the induction of DAX-1, SF-1, and StAR genes $(D-F)$ that closely matched the in vivo data. Similarly, the model approximated the expression of the gene products, as judged from the dynamics of mRNA $(G-l)$ and protein levels $(J-L)$. Red dashed lines indicate the time of ACTH injection. Representative Western immunoblotting images for pGR, StARp37, SF-1, and DAX-1 are shown in SI Appendix, Fig. S3.

ACTH also regulates steroidogenesis over a slightly slower time frame via modulation of the expression of genes involved in CORT synthesis. We therefore determined the effects of the i.v. ACTH injection on the dynamic expression of key steroidogenic genes in the adrenal SRN. Because many ACTH-regulated genes require activation of $C R E B$ to be transcribed, we first assessed the dynamics of CREB phosphorylation (pCREB). Consistent with previous reports $(29,30)$, there was a rapid and transient increase in pCREB (SI Appendix, Fig. S3D). We then investigated the dynamic transcription of steroidogenic genes by measuring their heteronuclear RNA (hnRNA) and mRNA levels. As expected, the dynamics of pCREB were paralleled by a transient activation of StAR hnRNA, followed by a slower StAR mRNA accumulation (Fig. $2 F$ and $I$ ). In response to the i.v. ACTH injection, there was a rapid, transient decrease in DAX-1 hnRNA and a dynamic increase in SF-1 hnRNA (Fig. $2 D$ and $E$ ). In contrast, no significant changes in DAX-1 and SF-1 mRNA (Fig. $2 G$ and $H$ ), and in DAX-1, SF-1, and StARp37 protein levels were detected within $2 \mathrm{~h}$ of ACTH administration (Fig. $2 J-L$ ).

With respect to those SRN factors that were not considered in the model, we found that the i.v. ACTH injection also had a rapid effect on the activity of HSL, a protein that plays a key role in the SRN by regulating intracellular metabolism of cholesterol (SI Appendix, Fig. S3C). We assessed this by measuring the phosphorylation dynamics at sites known to increase HSL activity (pHSL at Serine 660 and 563) (31) and observed a dynamic activation of both pHSL-(Ser660) and pHSL-(Ser563). The pHSL dynamics suggest that a rapid, transient increase in intracellular free cholesterol precedes CORT synthesis and supports previous findings that phosphorylation of HSL is a crucial step in the steroidogenic process. Furthermore, ACTH rapidly activated hnRNA and mRNA levels of MC2R, MRAP, Nur77, and CYP11A1, but had no effect on HSL hnRNA and mRNA (SI Appendix, Fig. S3E). No significant changes in CYP11A1, StARp32, StARp30, or HSL protein levels were detected within $2 \mathrm{~h}$ of ACTH administration (SI Appendix, Fig. S3F). Taken together, these results show that dynamic changes within both nongenomic and genomic processes are involved in the dynamic regulation of steroidogenesis.

Next, we fitted the model to the experimental data (Fig. 2). In addition to recalibrating model parameters, we further considered a potential posttranscriptional regulatory mechanism: an ACTH dose-dependent control of DAX-1 mRNA stability that accounts for the DAX-1 mRNA levels of varying magnitude observed following ACTH stimuli (32) (SI Appendix, Mathematical Model and Fig. S4). The time evolution of the SRN was generated by driving the mathematical model with a virtual ACTH pulse given during the nadir of the circadian rhythm (SI Appendix, Fig. S2 and Mathematical Model). These simulations showed that the A-CORT dynamics exhibited a rising phase at $<5 \mathrm{~min}$, a peak at $\sim 10 \mathrm{~min}$, and a falling phase at $>15$ min (Fig. $2 B$ ). Similarly, the pGR dynamics predicted by the model displayed a rising phase at $<5 \mathrm{~min}$, 
a peak phase at $\sim 12 \mathrm{~min}$, and a falling phase at $>15 \mathrm{~min}$ (Fig. $2 C$ ). The model also predicted a transient induction of the SF-1 and StAR genes, as judged from their rapidly inducible primary transcripts (hnRNA), mRNA, and protein dynamics (Fig. $2 E, F, H, I$, $K$, and $L$ ). In contrast, the model predicted a transient inhibition of DAX-1 hnRNA, but a transient induction of its mRNA and protein levels (Fig. $2 D, G$, and $J$ ). Taken together, these simulations closely matched the in vivo data for A-CORT and pGR dynamics, as well as gene induction (Fig. $2 B-F$ ). Although the changes in mRNA and protein levels measured in vivo were not statistically significant, our model still reproduced the transient dynamics of DAX-1 mRNA and protein (Fig. $2 G$ and $J$ ) and predicted a gradual increase of SF-1 and StAR mRNA and protein (Fig. $2 \mathrm{H}, \mathrm{I}$, $K$, and $L$ ) that is consistent with the transient increase of their corresponding hnRNAs. Discrepancies between the model and experimental data may originate from unexplored posttranscriptional or posttranslational regulatory mechanisms of SF-1 and StAR expression that the model does not account for.

Dynamic Responses of the SRN to Large ACTH Perturbations. Our data above show that an ACTH stimulus comparable to an endogenous ultradian pulse leads to dynamic changes within the SRN, ultimately driving the tightly correlated dynamic release of CORT. However, the adrenal gland is also subjected to more forceful perturbations (e.g., those associated with acute stressors), and in some cases the tight correlation between ACTH and
CORT is lost. This "dissociation" has been implicated in a change of the adrenal's sensitivity to ACTH (reviewed in ref. 6), suggesting that the SRN has the ability to decode ACTH signals in a contextdependent manner. Motivated by this, we used our model to investigate how the SRN responds to a larger ACTH perturbation, comparable to that measured in response to an inflammatory stress (8), and tested our model predictions experimentally.

We have previously developed an experimental methodology to apply a large ACTH stimulus to the rat adrenal glands (8). This consists of four sequential s.c. injections of ACTH at 35-min intervals, which results in a large surge in ACTH plasma concentrations comparable to that observed during inflammatory stress (Fig. 3A). To explore how such a substantial ACTH perturbation affects the dynamics of components within the adrenal SRN, we drove our model with this same pattern of ACTH stimulation and simulated the transient dynamics of CORT and pGR, as well as DAX-1, SF-1, and StAR. Our model predicted a rapid, transient increase in A-CORT consisting of a rising phase ( $<30 \mathrm{~min})$, a plateau (45-120 min), and a falling phase (>120 min) (Fig. 3B). As expected, the pGR dynamic profile predicted by the model was consistent with that of A-CORT (Fig. 3C). In terms of gene expression, the model predicted a transient inhibition of DAX1 gene expression, as well as a transient induction of SF-1 and StAR genes (Fig. $3 D-I$ ). Regarding protein levels, our model produced a transient activation of SF-1 and StARp37 and a gradual decline in DAX-1 (Fig. $3 J-L$ ).
A
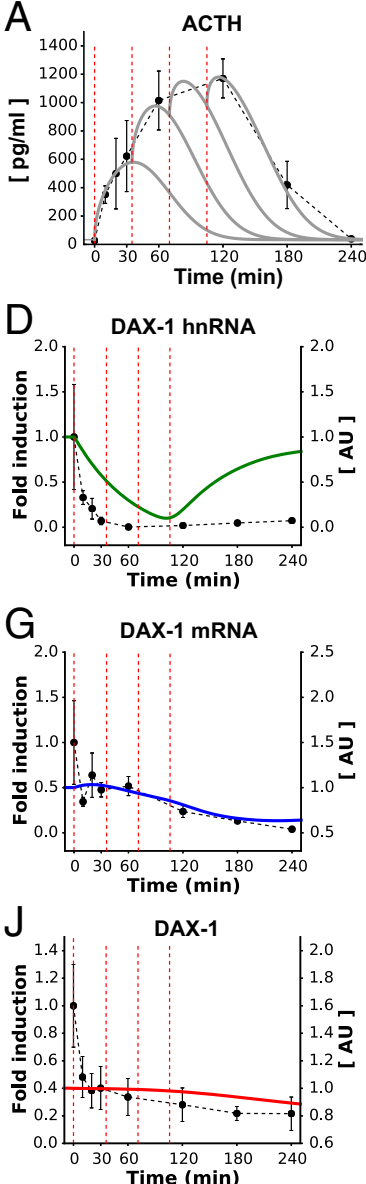
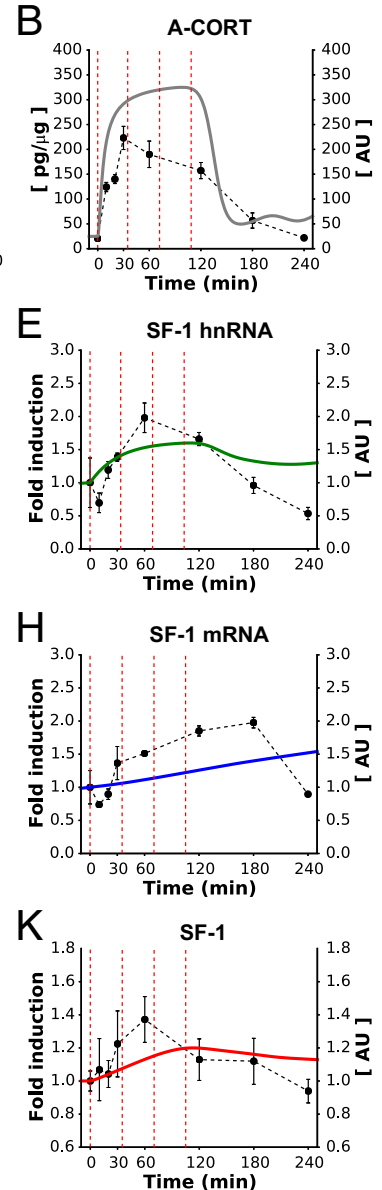
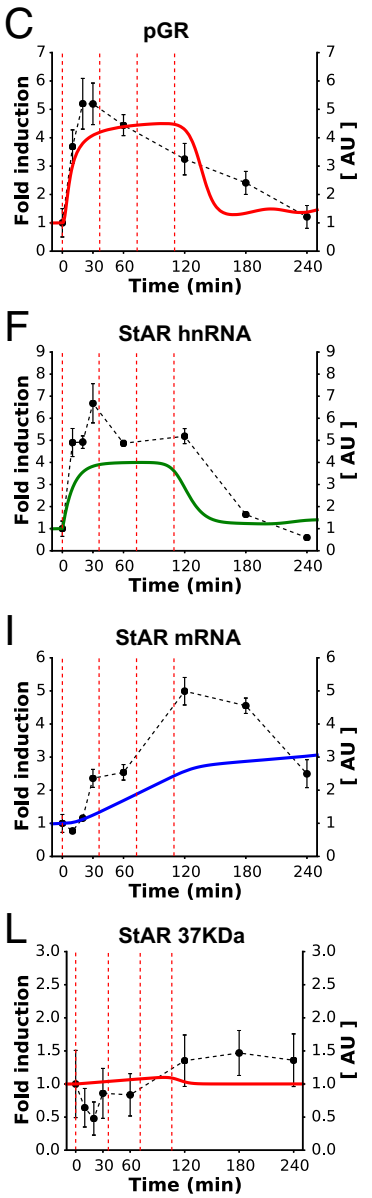

Fig. 3. The model reproduced the SRN dynamics following a high dose of ACTH $(A)$. The model successfully reproduced the long and transient surges of $A-C O R T$ ( $B$ ) and GR activation $(C)$ and also made qualitative predictions about the induction of DAX-1, SF-1, and StAR genes (D-F) that closely matched the in vivo data. Similarly, the model approximated the expression of the gene products, as judged from the dynamics of mRNA (G-l) and protein levels $(J-L)$. Red dashed lines indicate the time of ACTH injections. Representative Western immunoblotting images for pGR, StARp37, SF-1, and DAX-1 are shown in SI Appendix, Fig. S5. 
To test these modeling predictions, we measured the dynamic response of the rat SRN to this ACTH challenge. The increase in plasma ACTH levels was paralleled by a robust increase in both A-CORT (Fig. 3B) and P-CORT (SI Appendix, Fig. S5B). As observed in the i.v. ACTH pulse experiment, there was a dynamic increase in pGR that was consistent with the observed pattern of A-CORT (Fig. 3C). Consistent with the ACTH i.v. pulse data, there was a significant decrease in DAX-1 hnRNA and mRNA (Fig. $3 \mathrm{D}$ and $G$ ) and a significant increase in SF-1 and StAR hnRNA and mRNA (Fig. $3 E, F, H$, and $I$ ). With regard to protein levels, the high dose of ACTH also induced a significant increase in StARp30 (SI Appendix, Fig. S5F), but not in StARp37 (Fig. 3L), StARp32 (SI Appendix, Fig. S5F), or SF-1 (Fig. 3K). There was a nonsignificant decrease in DAX-1 protein (Fig. $3 J$ ). The dynamic effects of a high dose of ACTH on A-CORT were paralleled by an increase in both pHSL-(Ser660) and pHSL-(Ser563) (SI Appendix, Fig. S5C). Furthermore, dynamic transcription of StAR was accompanied by dynamic changes in pCREB (SI Appendix, Fig. S5D), as well as a rapid increase in MRAP, Nur77, and CYP11A1 hnRNA and mRNA. Unexpectedly, we observed a decrease in MC2R hnRNA and mRNA, and small dynamic changes were also observed for HSL hnRNA and mRNA (SI Appendix, Fig. S5E). In contrast, there was no effect on CYP11A1 and HSL protein (SI Appendix, Fig. S5F).

Overall, there was a good qualitative fit between our model predictions and our experimental data for both small and large
ACTH perturbations (Figs. 2 and 3, respectively). Importantly, the network architecture and parameter values in our SRN model were identical in both cases. This suggests that, for the majority of the molecular species considered in the model, no new mechanisms controlling the SRN dynamic response have to be introduced to explain the dynamic SRN responses to ACTH stimuli of varying magnitude.

Dynamic Responses of the SRN to an LPS Challenge. Our mathematical model of the SRN suggests that the adrenal response to ACTH is primarily dependent on the specific pattern of ACTH stimulation. However, in several stress scenarios, the adrenal can be influenced by other additional factors that modulate the SRN response. For example, activation of the immune system by administration of the bacterial endotoxin LPS is known to induce CORT secretion through a robust activation of the HPA axis, as well as through a direct effect at the level of adrenocortical steroidogenic cells (33). Therefore, we investigated the dynamic pattern of adrenal activation in response to such a stimulus. As previously shown (8), LPS administration induced an elevated and prolonged ACTH response (Fig. $4 A$ ) that was followed by a long-lasting A-CORT (Fig. 4B) and P-CORT (SI Appendix, Fig. $\mathrm{S} 6 B)$ response. Unexpectedly, despite the high levels of A-CORT measured after the LPS injection, there was no significant effect of LPS on pGR (Fig. 4C). An elevated and long-lasting effect of
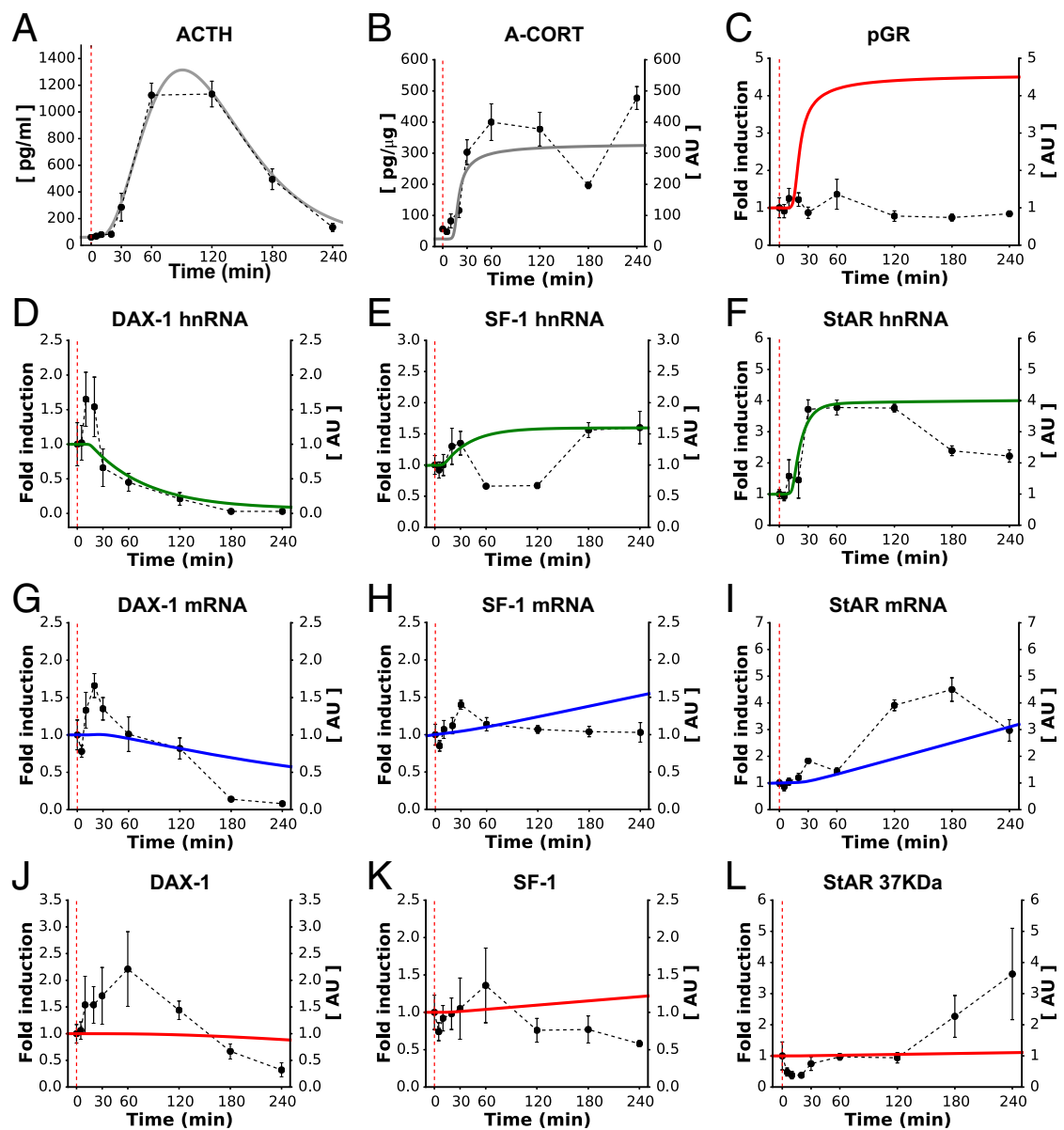

Fig. 4. Before considering the cross-talk with the immune pathway, the model partially reproduced the dynamic effects of an LPS-induced pulse of ACTH ( $A$ ) on components of the SRN. Although it ignores cytokine effects, the model successfully reproduced the long and sustained surge of $A-C O R T$ ( $B$ ), but not of GR activation $(C)$. The model also made qualitative predictions about the gene expression dynamics that closely matched the $D A X-1$ in vivo data $(D, G$, and $J)$, but failed to reproduce the SF-1 $(E, H$, and $K)$ and StAR $(F, I$, and $L)$ gene expression dynamics. Red dashed lines indicate the time of LPS injection. Representative Western immunoblotting images for pGR, StARp37, SF-1, and DAX-1 are shown in SI Appendix, Fig. S6. 
LPS was observed on the genomic adrenal steroidogenic pathway, including StAR hnRNA and mRNA (Fig. $4 F$ and $I$ ), and a multiphasic effect on SF-1 hnRNA (Fig. 4E) with a small nonsignificant increase in SF-1 mRNA (Fig. 4H). In contrast to the decrease in DAX-1 observed in the high-ACTH dose experiment, we observed a biphasic dynamic effect on DAX-1 hnRNA and mRNA (Fig. $4 D$ and $G$ ). We also measured the effects of LPS on protein levels and found that it increased the levels of StARp37 (Fig. $4 L$ ) and StARp32 (SI Appendix, Fig. S6F), and caused a nonsignificant increase in StARp30 (SI Appendix, Fig. S6F). There was no effect of LPS on SF-1 protein (Fig. $4 K$ ), but we observed a biphasic effect of LPS on DAX-1 protein (Fig. $4 J$ ) that was consistent with DAX-1 hnRNA and mRNA.

In parallel to the effect on CORT, there was a robust and prolonged effect on pHSL-(Ser660) and pHSL-(Ser563), as well as dynamic changes in pCREB (SI Appendix, Fig. S6 $C$ and D). Consistent with the findings from the high-ACTH dose experiment, LPS induced dynamic changes in MRAP, Nur77, and CYP11A1 hnRNA and mRNA and a significant decrease in MC2R and HSL hnRNA and mRNA (SI Appendix, Fig. S6E). Despite its effects on transcription, there was no effect of LPS on CYP11A1 and HSL protein (SI Appendix, Fig. S6F).

Injection of LPS in the rat is also known to increase plasma cytokines in a time-dependent manner (34). In addition, because LPS can also increase intra-adrenal expression of cytokines, we investigated the dynamics of intra-adrenal cytokines in LPStreated rats. As expected, LPS induced a rapid and transient increase in IL-1 $\beta$ hnRNA and mRNA, IL-6 hnRNA and mRNA, and TNF- $\alpha$ hnRNA and mRNA (SI Appendix, Fig. S7 $A-C$ ).

Although LPS increases both ACTH and cytokine levels, it is not clear whether these cytokines modulate the SRN response to $\mathrm{ACTH}$ and the mechanisms through which this may occur. To investigate this, we drove our model of the SRN with the ACTH pattern generated by the LPS injection and compared its output with the experimental data (Fig. $4 A$ ). Our model roughly approximated the dynamics of A-CORT and the DAX-1 hnRNA (Fig. $4 B$ and $D$ ). In contrast, the expression dynamics of SF-1 and StAR showed some discrepancies in their hnRNA dynamics (Fig. $4 E$ and $F$ ), where the model failed to reproduce the biphasic induction of these genes. Parallel to A-CORT, the model predicted induction and sustained activation of pGR. This was in stark contrast with our experimental data showing a lack of pGR activation despite high A-CORT (Fig. 4C). Taken together, these results suggest that a model of the SRN lacking cytokine regulation is not sufficient to explain its response to LPS.

Dynamic Effects of LPS-Induced Adrenal Cytokine Expression on the SRN. The lack of GR activation in response to LPS, together with the fact that LPS has been shown to repress GR function in other tissues (reviewed in ref. 35), points to an interaction between cytokines and GR. Indeed, previous findings show that the LPSinduced proinflammatory cytokine TNF- $\alpha$ inhibits GR phosphorylation in Serine 211 in human airway cells (36). In light of this, we extended our model to account for cross-talk between components of the SRN and the immune pathway, and used it to test whether these interactions could account for the observed response to LPS. To do this, we constructed a map of the network architecture to elucidate its effects upon steroidogenesis (SI Appendix, Fig. S8 and Mathematical Model). As was the case for the full steroidogenic network, we simplified the map of interactions between the immune pathway and the SRN (Fig. 5 and SI Appendix, Mathematical Model). Furthermore, our timecourse data on cytokine mRNA levels measured in the adrenals exhibited a timescale similar to cytokine protein dynamics in plasma following LPS stimulation (34). Thus, we accounted for the effects that cytokines TNF- $\alpha$, IL- $1 \beta$, and IL-6 have upon specific components of the SRN by using their mRNA timecourse data. In other words, in addition to the high-ACTH response elicited by LPS, we used the cytokines' dynamic profiles as additional, selective input to specific targets within the SRN (SI Appendix, Fig. S9).

We modified the mathematical model to account for these cytokine effects (SI Appendix, Mathematical Model) and carried out computer simulations to explore the steroidogenic response to LPS. The mathematical model predicted not only the sustained induction of A-CORT (Fig. $6 B$ ), but also the inhibition of pGR that was observed following LPS injection (Fig. $6 C$; compare with Fig. $4 C$ ). The model also predicted the transient, multiphasic induction of SF-1 and StAR genes (Fig. $6 E$ and $F$ ) as well as the transient inhibition of DAX-1 gene expression (Fig. 6D) and SF-1, StAR, and DAX-1 mRNA and protein dynamics (Fig. $6 G-L$ ).

\section{Discussion}

Previous studies of adrenal steroidogenesis have focused on specific interactions between genes and proteins involved in glucocorticoid synthesis. Although these studies have provided important biological insight into the structure and regulation of key steroidogenic factors, the dynamics of the interactions of these factors across different timescales has not been considered. Indeed, using static data on individual interactions within the steroidogenic pathway has not been sufficient to explain how specific patterns of ACTH stimulation are translated by the adrenals into specific patterns of CORT secretion.

To understand this, we explored how some of these factors interact with one another in the context of a complex dynamic regulatory network. Because of the dynamic nature of steroidogenesis, we developed a mathematical model of the SRN that accounted for regulatory processes occurring at different timescales. Importantly, we also considered an intra-adrenal CORTmediated feedback loop, the effects of which were previously described through mathematical modeling (17). We used our model to predict the time evolution of steroidogenesis following a series of physiological perturbations relevant to stress and inflammation. By testing these predictions experimentally, we have shown how the SRN can respond to both small and large ACTH perturbations and how the coupling with the immune pathway may be necessary to elicit an ACTH/CORT-dissociated response during inflammation.

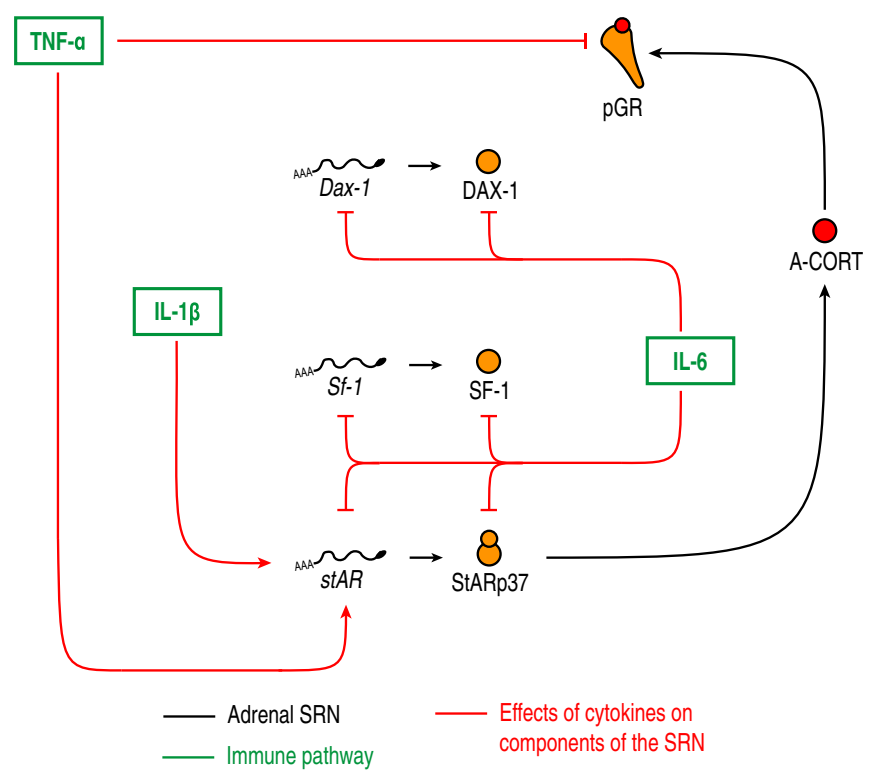

Fig. 5. Cytokine effects upon targets within the adrenal SRN considered in the model. The transient cytokine pulses elicited by LPS were used as additional input functions to ACTH. 

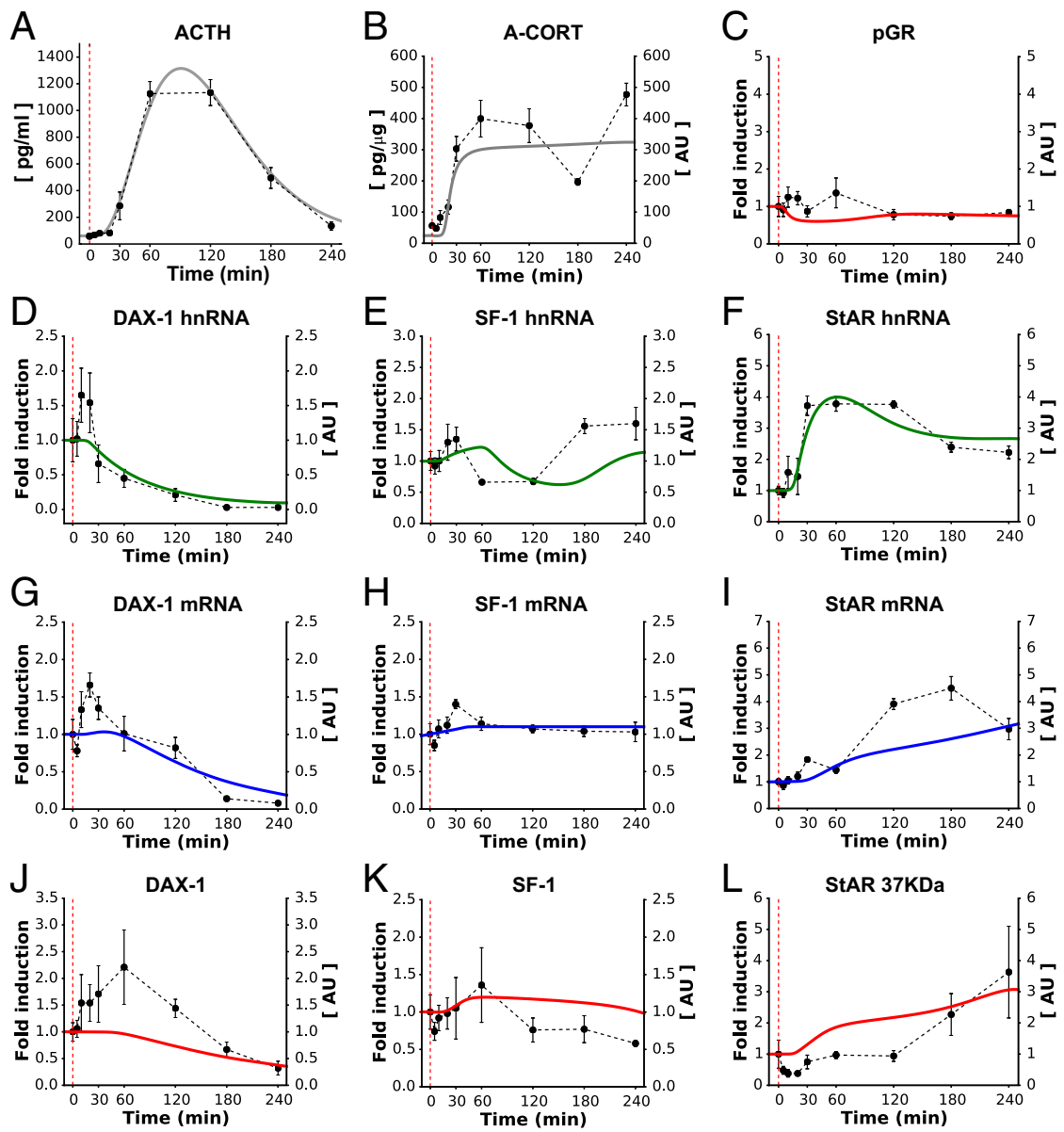

Fig. 6. The extended model reproduced the SRN dynamics following administration of LPS. After accounting for both ACTH ( $A$ ) and cytokine effects, the model successfully reproduced the long and sustained surge of A-CORT $(B)$ and approximated the pGR dynamics ( $C$ ). The model also made qualitative predictions about the transient induction of DAX-1, SF-1, and StAR genes $(D-F)$ that approximated the in vivo data. Similarly, the model approximated the expression of the gene products, as judged from the dynamics of mRNA (G-l) and protein levels ( $J-L)$. Red dashed lines indicate the time of LPS injection. Representative Western immunoblotting images for pGR, StARp37, SF-1, and DAX-1 are shown in SI Appendix, Fig. S6.

Mathematical models of CORT dynamics have been developed to study the origin of ultradian oscillations within the HPA axis (4, $17)$ and the inflammatory response at the adrenal level (37). However, these models do not consider the dynamic molecular interactions within adrenocortical cells that occur during steroidogenesis. In this study, we used a mathematical modeling approach that focuses on the adrenal cell's network architecture, the interplay between fast and slow processes, and posttranscriptional and posttranslational control of steroidogenic genes. Predictions from this model can only be of a qualitative nature, which reflects our limited knowledge of the regulatory processes that we modeled phenomenologically (e.g., using Michaelis-Menten and Hill-type functions) and the uncertainties associated with some parameter values. Another limitation relates to the fact that our model did not consider all of the components within the full SRN. This reflects the need to simplify our computational analysis by reducing the network architecture to its core components. Nonetheless, our results show that the SRN operates with the same core components, network architecture, and parameter values to respond to both small and large ACTH perturbations; in other words, no new mechanisms need to be introduced to explain the adrenal's response to a range of ACTH stimuli.

We observed that rapid and transient CORT responses to ACTH stimulation are accompanied by dynamic changes in several components within the adrenal SRN. Previously, we have shown that ultradian secretion of corticosterone in the rat is paralleled by pulsatile transcription of steroidogenic genes including StAR, CYP11A, and MRAP and pulsatile phosphorylation of CREB $(29,30,38)$, suggesting that dynamic activation of the adrenal steroidogenic pathway is important for maintaining ultradian glucocorticoid secretion. In this study, we aimed to further elucidate how ACTH regulates adrenocortical dynamics in response to a pulse of ACTH. We have provided evidence of ultradian responsiveness at the level of nongenomic mechanisms within the adrenal steroidogenic pathway (i.e., phosphorylation of HSL) and within the transcriptional regulation of steroidogenic gene regulators (i.e., transcription of SF-1, Nur77, and DAX-1). These findings suggest that ultradian secretion of ACTH is important not only for a rapid "acute" adrenal response, but also for long-term maintenance of the steroidogenic pathway under basal conditions. Overall, our data suggest that dynamic events occurring within the adrenal SRN are important in maintaining ultradian secretion of glucocorticoids in a normal, healthy state.

Although there was a good fit between the model and the hormone, hnRNA and pGR data, some discrepancies were observed for mRNA and protein. The levels of mRNA and protein are typically thought to depend on the transcription of the gene, but additional regulatory factors such as RNA-binding proteins and microRNAs can affect the stability of transcripts. Because a role for ACTH-dependent regulation of mRNA stability in the adrenal cortex has been proposed (reviewed in ref. 39), we 
hypothesize that mechanisms such as these could explain the discrepancies we have observed.

Recent data from our group suggest that, in addition to the well-known CORT-mediated negative feedback at the pituitary and brain regulating HPA axis activity, an intra-adrenal negative feedback involving activation of the GR can rapidly inhibit ACTH-induced glucocorticoid synthesis (17). Consistent with this, there is evidence that glucocorticoids can inhibit the transcription of steroidogenic genes through a mechanism that involves the synergistic association of SF-1 and GR to modulate DAX-1 expression (20). The present study provides further evidence to support this hypothesis by showing that a rapid, transient activation of adrenal GR tightly follows the dynamics of intra-adrenal corticosterone.

Further implications of an intra-adrenal CORT negative feedback loop relate to the role that the DAX-1, SF-1, and StAR genes play in controlling CORT synthesis. For example, although DAX-1 expression is critical for normal HPA organ development $(40-42)$, its role in regulating steroidogenesis in the adult is still unclear. SF-1 and GR have been shown to synergistically modulate DAX-1 expression (20), which in turn inhibits SF-1-mediated induction of StAR (43). ACTH is known to disrupt this SF-1/GR synergistic effect on DAX-1 (20), and GR is activated by intraadrenal CORT. This suggests that DAX-1 may be acting as an organizing "hub" by simultaneously integrating and decoding hormone signals from both the pituitary and the adrenal to control steroidogenesis. If this is the case, it is possible that changes in the way this "hub" functions could lead to alterations in the way the adrenal SRN responds to ACTH perturbations.

We also studied the steroidogenic response to an LPS injection. In this case, not only the SRN but also the immune pathway was activated, as shown by the induction of cytokines TNF- $\alpha$, IL- 6 , and IL-1 $\beta$. The cross-talk between the steroidogenic and immune pathways has been modeled previously, although only at the system level (37). The mathematical model that we present here is the first to incorporate a detailed description of the SRN and its interactions with the immune pathway. Notably, our model failed to reproduce the LPS response until we explicitly accounted for the effects that cytokines have upon specific components within the SRN. This suggests that cytokines interact with ACTH to mediate the inflammatory response at the level of the adrenal SRN. Although it has been shown that a severe stressor can increase cytokine levels in the brain and pituitary even in the absence of inflammation (44), and that ACTH can increase cytokine secretion in the adrenal gland in vitro $(45,46)$, we did not see such an increase in the level of adrenal cytokines following a high-ACTH stimulation alone.

A surprising finding of our study is the biphasic change in DAX-1 hnRNA, mRNA, and protein observed in the LPS experiment. Although it is known that IL-6 down-regulates DAX-1 gene expression in bovine zona fasciculate cells (47), studies in different steroidogenic tissues have shown that TNF- $\alpha$ can increase the expression of DAX-1 protein (48). Indeed, a rapid increase in TNF- $\alpha$ levels in plasma has been observed shortly after LPS stimulation in the rat (34), and we have observed a rapid increase in TNF- $\alpha$ hnRNA and mRNA in this study. It is therefore tempting to speculate that a transient enhancement of DAX-1 in response to increased TNF- $\alpha$ may occur in the zona fasciculata of rats injected with LPS before the decrease induced by IL-6.

The sustained CORT response to transient ACTH following an LPS injection suggests a disruption in the GR-mediated intraadrenal feedback loop. This could be a factor underlying the dissociated dynamics between ACTH and CORT observed in stress scenarios. Uncovering the origin of these dissociated dynamics is key to understanding a number of physiological and pathological conditions where sustained high levels of CORT have been observed even after ACTH has returned to normal (8). Consistent with this, our model predicts that cytokine effects on the DAX-1 regulatory "hub" of the SRN may explain the dynamic dissociation between ACTH and CORT during inflammation.

With regard to other measured factors that were not included in the model, on the whole, we observed a dynamic activity that was consistent with the ACTH and/or LPS stimulation. However, we did note some discrepancies with previous studies; for example, it has been shown that ACTH induces the expression of MC2R (25). However, in our study we found that, although a small pulse of ACTH increased MC2R hnRNA and mRNA, administration of LPS decreased MC2R hnRNA and mRNA. Similarly, although a pulse of ACTH had no effect on HSL expression, LPS decreased both HSL hnRNA and mRNA. This suggests that inflammatory stress can lead to a decrease in ACTH signaling and cholesterol availability, which may in turn lead to adrenal hypo-responsiveness in the longer-term that is often observed in chronic inflammation and sepsis (49).

In summary, we have presented a mathematical model of steroidogenesis that makes qualitative predictions in response to a range of $\mathrm{ACTH}$ stimuli. Further in vitro experiments are required to shed light on the specific molecular mechanisms regulating the cross-talk between steroidogenic genes, which would enable us to refine our models to make quantitative predictions. Nonetheless, we have shown that our mathematical model of the adrenal SRN delivers valuable insight about the transient and rapid adrenal dynamics observed in response to ACTH perturbations during both basal and acute stress scenarios. In future work, the model could be further exploited to study several ACTH-independent mechanisms regulating adrenal steroidogenesis, such as modulation by the splanchnic nerve (50), the influence of metabolic factors such as leptin and insulin (6), and the effects of acute or chronic exposure to synthetic glucocorticoids.

\section{Materials and Methods}

Animals and Surgery. All experiments were conducted on adult male SpragueDawley rats (Harlan Laboratories) weighing 220-250 g at the time of arrival.

Rats were anesthetized using isoflurane, and an indwelling catheter was inserted in the right jugular vein as previously described (51).

Experiments and Tissue Collection. All experiments started at 9 AM and were performed 5-7 d after the surgery. Rats were given the following: (i) an i.v. injection of synthetic ACTH (10 ng/0.1 mL; ACTH pulse experiment); (ii) four s.c. injections of ACTH $(2 \mu \mathrm{g} / \mathrm{kg}$; high-dose ACTH experiment; and (iii) an i.v. injection of LPS $(25 \mu \mathrm{g}$ per rat in $0.1 \mathrm{~mL}$ of sterile saline; LPS experiment).

After decapitation, trunk blood was collected and plasma processed for ACTH and corticosterone measurement as previously described (51). Adrenal glands were collected and the inner zones (comprising the zona fasciculata and the zona reticularis of the cortex and the adrenal medulla) were separated from the outer zone (containing the zona glomerulosa and the capsula). Individual inner zones were immediately frozen until processing for isolation of RNA for real-time quantitative PCR (left adrenal) and for protein extraction for Western immunoblotting and corticosterone measurement (right adrenal) as previously described $(38,52)$.

Statistical Analysis. Data were analyzed using the nonparametric KruskalWallis Test. Statistical significance was set at $P<0.05$.

Mathematical Model. We developed a mathematical model of the adrenal SRN that accounts for the time evolution of core components of the full network, namely A-CORT, pGR, and the genes DAX-1, SF-1, and StAR. The mode postulates a network architecture based on known biological interactions with parameter values estimated from the literature and calibrated using the ACTH i.v. pulse data. The network architecture and kinetic parameter values remained invariant for generating predictions about the high s.C. ACTH and LPS challenge experiments.

Because of the involvement of a slow genomic pathway and a fast nongenomic one, we wrote the model in terms of a set of DDEs, where the delays were associated with the gene transcription and translation processes. Our set of nonlinear, coupled DDEs was numerically integrated using XPPAUT (53) and PyDDE with a fixed-time step of $0.001 \mathrm{~min}$ and a fourth-order Runge-Kutta 
integrator with adaptive steps. The model development and complete set of model equations are detailed in SI Appendix, Mathematical Model.

More details on materials and methods can be found in SI Appendix, Supplementary Materials and Methods. Access to data, associated protocols, code, and materials is available upon reasonable request.

1. Ixart G, et al. (1994) Short-term but not long-term adrenalectomy modulates amplitude and frequency of the $\mathrm{CRH} 41$ episodic release in push-pull cannulated median eminence of free-moving rats. Brain Res 658:185-191.

2. Ixart G, Barbanel G, Nouguier-Soulé J, Assenmacher I (1991) A quantitative study of the pulsatile parameters of $\mathrm{CRH}-41$ secretion in unanesthetized free-moving rats. Exp Brain Res 87:153-158.

3. Walker JJ, et al. (2012) The origin of glucocorticoid hormone oscillations. PLoS Biol 10: e1001341.

4. Walker JJ, Terry JR, Lightman SL (2010) Origin of ultradian pulsatility in the hypothalamic-pituitary-adrenal axis. Proc Biol Sci 277:1627-1633.

5. Henley DE, et al. (2009) Development of an automated blood sampling system for use in humans. J Med Eng Technol 33:199-208.

6. Bornstein SR, Engeland WC, Ehrhart-Bornstein M, Herman JP (2008) Dissociation of ACTH and glucocorticoids. Trends Endocrinol Metab 19:175-180.

7. Tkachenko IV, Jääskeläinen T, Jääskeläinen J, Palvimo JJ, Voutilainen R (2011) Inter leukins $1 \alpha$ and $1 \beta$ as regulators of steroidogenesis in human $\mathrm{NCl}-\mathrm{H} 295 \mathrm{R}$ adrenocortica cells. Steroids 76:1103-1115.

8. Gibbison B, et al. (2015) Dynamic pituitary-adrenal interactions in response to cardiac surgery. Crit Care Med 43:791-800.

9. Kraemer FB, Shen WJ (2002) Hormone-sensitive lipase: Control of intracellular tri-(di-) acylglycerol and cholesteryl ester hydrolysis. J Lipid Res 43:1585-1594.

10. Lin D, et al. (1995) Role of steroidogenic acute regulatory protein in adrenal and gonadal steroidogenesis. Science 267:1828-1831.

11. Caron KM, et al. (1997) Characterization of the promoter region of the mouse gene encoding the steroidogenic acute regulatory protein. Mol Endocrinol 11:138-147.

12. Martin LJ, Boucher N, Brousseau C, Tremblay JJ (2008) The orphan nuclear receptor NUR77 regulates hormone-induced StAR transcription in Leydig cells through cooperation with $\mathrm{Ca2}+/ \mathrm{calmodulin}-$ dependent protein kinase I. Mol Endocrinol 22 2021-2037.

13. Jo Y, Stocco DM (2004) Regulation of steroidogenesis and steroidogenic acute regulatory protein in R2C cells by DAX-1 (dosage-sensitive sex reversal, adrenal hypoplasia congenita, critical region on the X chromosome, gene-1). Endocrinology 145:5629-5637.

14. Carsia RV, Malamed S (1979) Acute self-suppression of corticosteroidogenesis in isolated adrenocortical cells. Endocrinology 105:911-914.

15. Loose DS, Do YS, Chen TL, Feldman D (1980) Demonstration of glucocorticoid receptors in the adrenal cortex: Evidence for a direct dexamethasone suppressive effect on the rat adrenal gland. Endocrinology 107:137-146.

16. Peron FG, Moncloa F, Dorfman RI (1960) Studies on the possible inhibitory effect of corticosterone on corticosteroidogenesis at the adrenal level in the rat. Endocrinology 67:379-388

17. Walker JJ, et al. (2015) Rapid intra-adrenal feedback regulation of glucocorticoid synthesis. $J R$ Soc Interface 12:20140875.

18. Briassoulis G, Damjanovic S, Xekouki P, Lefebvre H, Stratakis CA (2011) The glucocorticoid receptor and its expression in the anterior pituitary and the adrenal cortex A source of variation in hypothalamic-pituitary-adrenal axis function; implications for pituitary and adrenal tumors. Endocr Pract 17:941-948.

19. Chong C, et al. (2017) Regulation of aldosterone secretion by mineralocorticoid receptor-mediated signaling. J Endocrinol 232:525-534.

20. Gummow BM, Scheys JO, Cancelli VR, Hammer GD (2006) Reciprocal regulation of glucocorticoid receptor-steroidogenic factor-1 transcription complex on the Dax1 promoter by glucocorticoids and adrenocorticotropic hormone in the adrenal cor tex. Mol Endocrinol 20:2711-2723.

21. Babu PS, et al. (2002) Interaction between Dax-1 and steroidogenic factor-1 in vivo: Increased adrenal responsiveness to ACTH in the absence of Dax-1. Endocrinology 143:665-673.

22. Fan $\mathrm{W}$, et al. (2004) Protein kinase $A$ potentiates adrenal 4 binding protein/steroidogenic factor 1 transactivation by reintegrating the subcellular dynamic interactions of the nuclear receptor with its cofactors, general control nonderepressed-5/transformation transcription domain-associated protein, and suppressor, dosage-sensitive sex reversal-1: A laser confocal imaging study in living KGN cells. Mol Endocrinol 18:127-141.

23. Sugawara T, et al. (1997) Regulation of expression of the steroidogenic acute regulatory protein (StAR) gene: A central role for steroidogenic factor 1. Steroids 62:5-9.

24. Sugawara T, Kiriakidou M, McAllister JM, Kallen CB, Strauss JF, III (1997) Multiple steroidogenic factor 1 binding elements in the human steroidogenic acute regulatory protein gene 5 '-flanking region are required for maximal promoter activity and cyclic AMP responsiveness. Biochemistry 36:7249-7255.

25. Winnay JN, Hammer GD (2006) Adrenocorticotropic hormone-mediated signaling cascades coordinate a cyclic pattern of steroidogenic factor 1-dependent transcriptional activation. Mol Endocrinol 20:147-166.
ACKNOWLEDGMENTS. This work was funded by the Medical Research Council (MRC) Grant MR/J008893/1 (to F.S., E.Z., J.J.W., Z.Z., J.R.T., and S.L.L.) and Fellowship MR/N008936/1 (to J.J.W.); the Wellcome Trust Grant WT105618MA (to J.J.W. and J.R.T.); and the Engineering and Physical Sciences Research Council (EPSRC) Grant EP/N014391/1 (to J.J.W., J.R.T., and S.L.L.).

26. Carnes M, Brownfield MS, Kalin NH, Lent S, Barksdale CM (1986) Episodic secretion of ACTH in rats. Peptides 7:219-223.

27. Carnes $\mathrm{M}$, Kalin NH, Lent SJ, Barksdale CM, Brownfield MS (1988) Pulsatile ACTH secretion: Variation with time of day and relationship to cortisol. Peptides 9:325-331.

28. Wang Z, Frederick J, Garabedian MJ (2002) Deciphering the phosphorylation "code" of the glucocorticoid receptor in vivo. J Biol Chem 277:26573-26580.

29. Liu Y, et al. (2013) Transcriptional regulation of episodic glucocorticoid secretion. Mol Cell Endocrinol 371:62-70.

30. Spiga F, Liu Y, Aguilera G, Lightman SL (2011) Temporal effect of adrenocorticotrophic hormone on adrenal glucocorticoid steroidogenesis: Involvement of the transducer of regulated cyclic AMP-response element-binding protein activity. $J$ Neuroendocrinol 23 : $136-142$

31. Anthonsen MW, Rönnstrand L, Wernstedt C, Degerman E, Holm C (1998) Identification of novel phosphorylation sites in hormone-sensitive lipase that are phosphorylated in response to isoproterenol and govern activation properties in vitro. $J$ Biol Chem 273:215-221.

32. Ragazzon B, et al. (2006) Adrenocorticotropin-dependent changes in SF-1/DAX-1 ratio influence steroidogenic genes expression in a novel model of glucocorticoid-producing adrenocortical cell lines derived from targeted tumorigenesis. Endocrinology 147:1805-1818.

33. Zacharowski K, et al. (2006) Toll-like receptor 4 plays a crucial role in the immuneadrenal response to systemic inflammatory response syndrome. Proc Natl Acad Sci USA 103:6392-6397.

34. Givalois L, et al. (1994) Temporal cascade of plasma level surges in ACTH, corticosterone, and cytokines in endotoxin-challenged rats. Am J Physiol 267:R164-R170.

35. Marketon Jl, Sternberg EM (2010) The glucocorticoid receptor: A revisited target for toxins. Toxins (Basel) 2:1357-1380

36. Bouazza B, et al. (2012) Cytokines alter glucocorticoid receptor phosphorylation in airway cells: Role of phosphatases. Am J Respir Cell Mol Biol 47:464-473.

37. Malek H, Ebadzadeh MM, Safabakhsh R, Razavi A, Zaringhalam J (2015) Dynamics of the HPA axis and inflammatory cytokines: Insights from mathematical modeling Comput Biol Med 67:1-12

38. Spiga F, et al. (2011) ACTH-dependent ultradian rhythm of corticosterone secretion. Endocrinology 152:1448-1457.

39. Desroches-Castan A, Feige JJ, Cherradi N (2017) ACTH action on messenger RNA stability mechanisms. Front Endocrinol (Lausanne) 8:3.

40. Achermann JC, Meeks JJ, Jameson JL (2001) Phenotypic spectrum of mutations in DAX-1 and SF-1. Mol Cell Endocrinol 185:17-25.

41. Iyer AK, McCabe ER (2004) Molecular mechanisms of DAX1 action. Mol Genet Metab 83:60-73.

42. Zhao Y, et al. (2006) Zebrafish dax1 is required for development of the interrenal organ, the adrenal cortex equivalent. Mol Endocrinol 20:2630-2640.

43. Zazopoulos E, Lalli E, Stocco DM, Sassone-Corsi P (1997) DNA binding and transcriptional repression by DAX-1 blocks steroidogenesis. Nature 390:311-315.

44. Connor KA, et al. (2003) Inescapable shock induces resistance to the effects of dexamethasone. Psychoneuroendocrinology 28:481-500.

45. Judd AM, MacLeod RM (1992) Adrenocorticotropin increases interleukin-6 release from rat adrenal zona glomerulosa cells. Endocrinology 130:1245-1254.

46. Call GB, et al. (2000) Bovine adrenal cells secrete interleukin- 6 and tumor necrosis factor in vitro. Gen Comp Endocrinol 118:249-261.

47. Mcllmoil S, Strickland J, Judd AM (2016) Interleukin 6 increases the in vitro expression of key proteins associated with steroidogenesis in the bovine adrenal zona fasciculata. Domest Anim Endocrinol 55:11-24.

48. Sadasivam M, Ramatchandirin B, Balakrishnan S, Prahalathan C (2015) Tnf- $\alpha$-mediated suppression of Leydig cell steroidogenesis involves DAX-1. Inflammation Research 64: 549-556.

49. Boonen E, Bornstein SR, Van den Berghe G (2015) New insights into the controversy of adrenal function during critical illness. Lancet Diabetes Endocrinol 3:805-815.

50. Ulrich-Lai YM, Arnhold MM, Engeland WC (2006) Adrenal splanchnic innervation contributes to the diurnal rhythm of plasma corticosterone in rats by modulating adrenal sensitivity to ACTH. Am J Physiol Regul Integr Comp Physiol 290:R1128-R1135.

51. Spiga F, et al. (2007) Effect of the glucocorticoid receptor antagonist Org 34850 on basal and stress-induced corticosterone secretion. J Neuroendocrinol 19:891-900.

52. Park SY, et al. (2013) Constant light disrupts the circadian rhythm of steroidogenic proteins in the rat adrenal gland. Mol Cell Endocrinol 371:114-123.

53. Ermentrout B (2002) Simulating, Analyzing, and Animating Dynamical Systems: A Guide to XPPAUT for Researchers and Students (Siam, Philadelphia). 


\section{SI Appendix}

\section{Supplementary Figures}

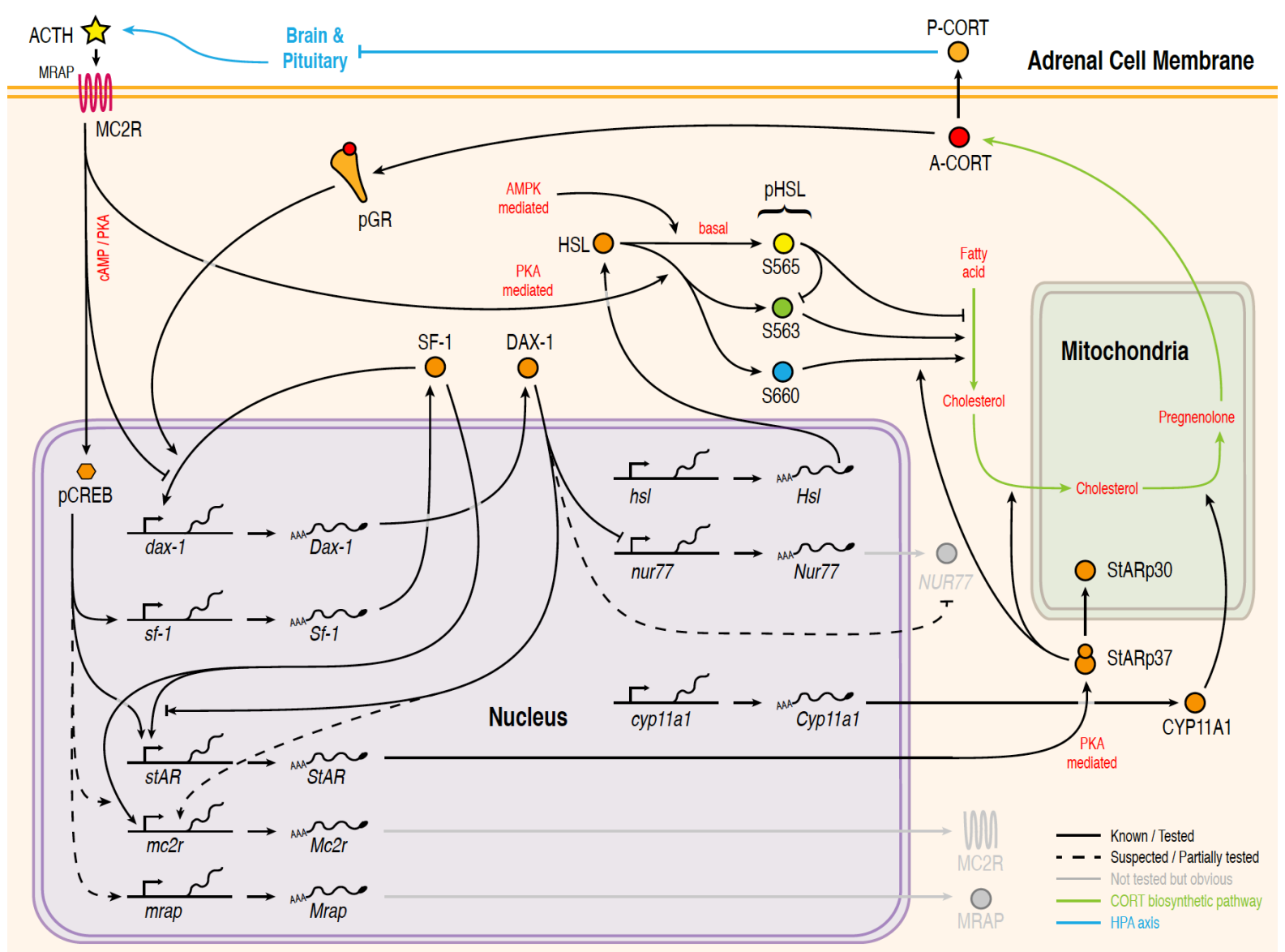

Fig. S1. The steroidogenic regulatory network (SRN). The synthesis of glucocorticoids in adrenocortical cells is governed at multiple levels by both genomic and non-genomic components. In addition to the high degree of cross-talk between these components, an intra-adrenal feedback loop mediated by A-CORT/GR may underlie adrenal responsiveness and maintain appropriate levels of glucocorticoids. 

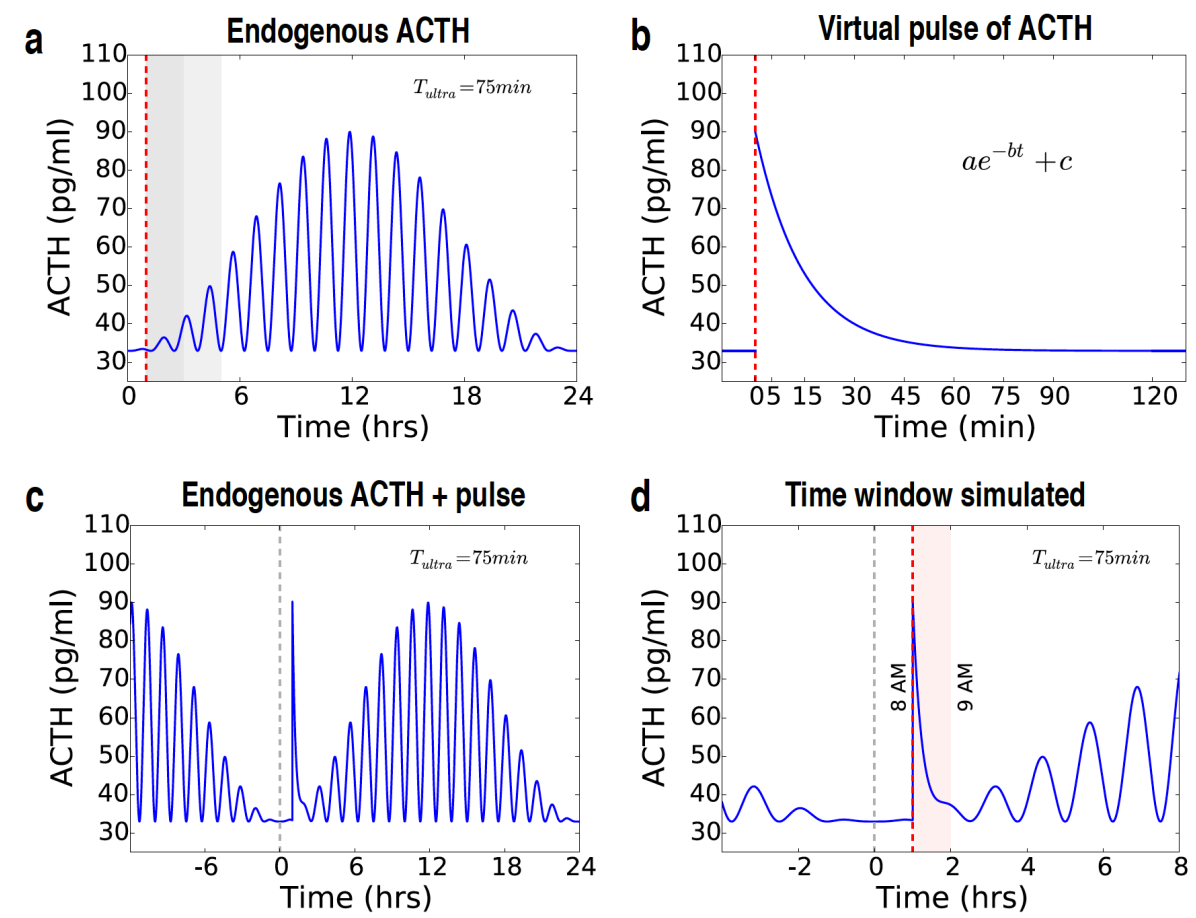

Fig. S2. Virtual (in silico) computer simulations of ACTH endogenous oscillations and perturbations. (a) The model was driven by oscillating levels of ACTH that represented endogenous fluctuations. (b) A simple function representing a "virtual" pulse of ACTH. (c) The pulse was of a size comparable to the maximum endogenous levels in normal physiological conditions. (d) The "virtual" pulse was given near the nadir of the circadian $\mathrm{ACTH}$ rhythm, at approximately the same time corresponding to the in vivo experiments (between 8 and 9 AM). 
a

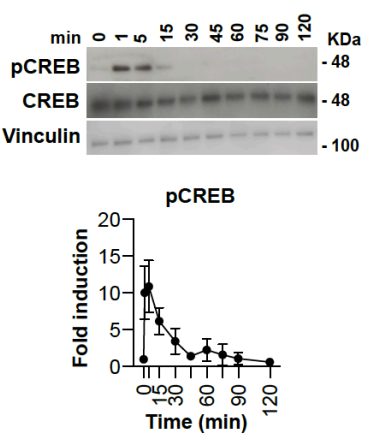

f

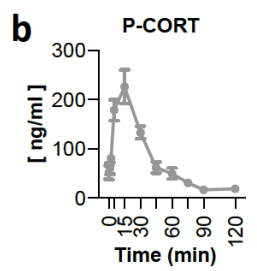

e

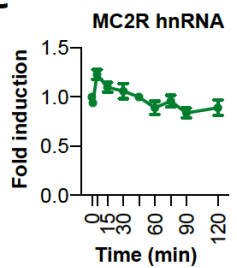

MC2R MRNA

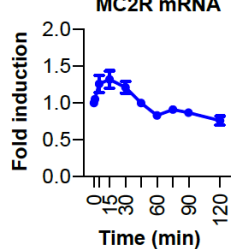

C

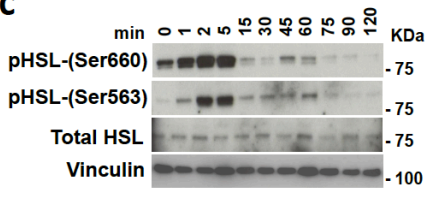

MRAP hnRNA
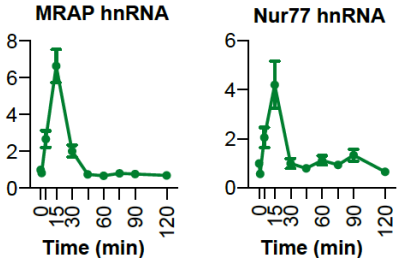

MRAP MRNA

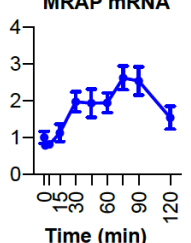

Nur77 mRNA

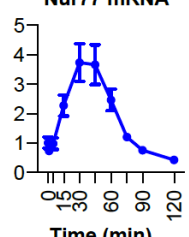

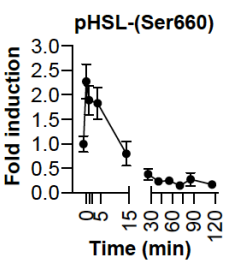
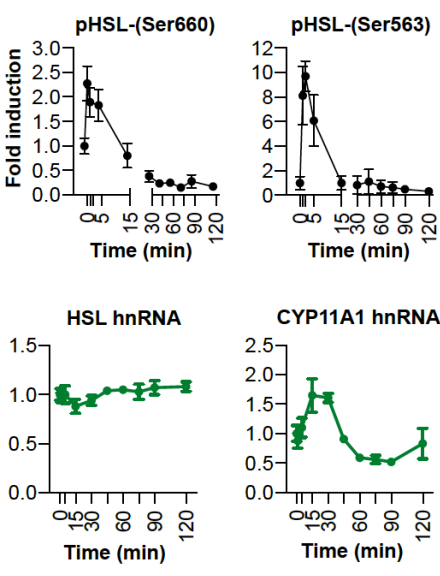

HSL mRNA

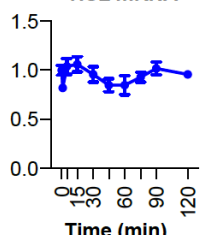

CYP11A1 hnRNA

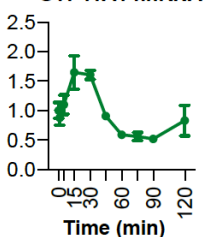

CYP11A1 mRNA

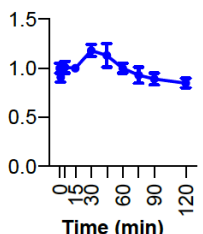

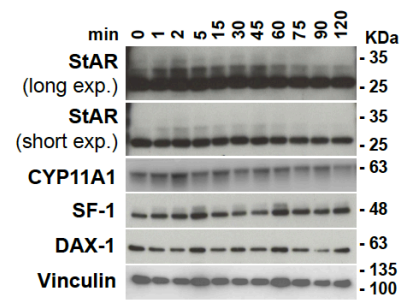
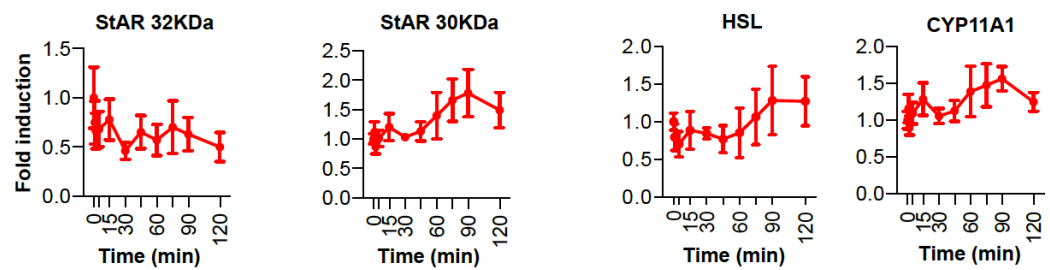

Fig. S3. A short pulse of ACTH dynamically activates the SRN and CORT biosynthesis. (a) Representative Western immunoblotting of the effect of a pulse of ACTH on intra-adrenal phospho-GR (quantification is shown in Fig. $2 \mathrm{c}$ in the main text). (b) A pulse of ACTH dynamically affects plasma CORT (P-CORT). (c) Representative Western immunoblotting and quantification of the dynamic effect of a pulse of ACTH on phosphorylation of HSL (pHSL-(Ser660) and pHSL-(Ser563)). (d) Representative Western immunoblotting and quantification of the dynamic effect of a pulse of ACTH on phosphorylation of CREB in Ser133. (e) A pulse of ACTH dynamically affects plasma CORT steroidogenic genes hnRNA and mRNA. (f) Representative Western immunoblotting and quantification of the dynamic effect of a pulse of ACTH on steroidogenic protein expression (quantification of StARp37, SF-1 and DAX-1 proteins is shown in Fig. 2 in the main text). For Western immunoblotting data, optical density was normalised to Vinculin; for RTqPCR, data were normalised to GAPDH mRNA levels; Western immunoblotting and RTqPCR data are expressed as fold induction of time 0 ( $n=4-7 /$ time point). Details of asymptotic significances are reported in Table S2. 


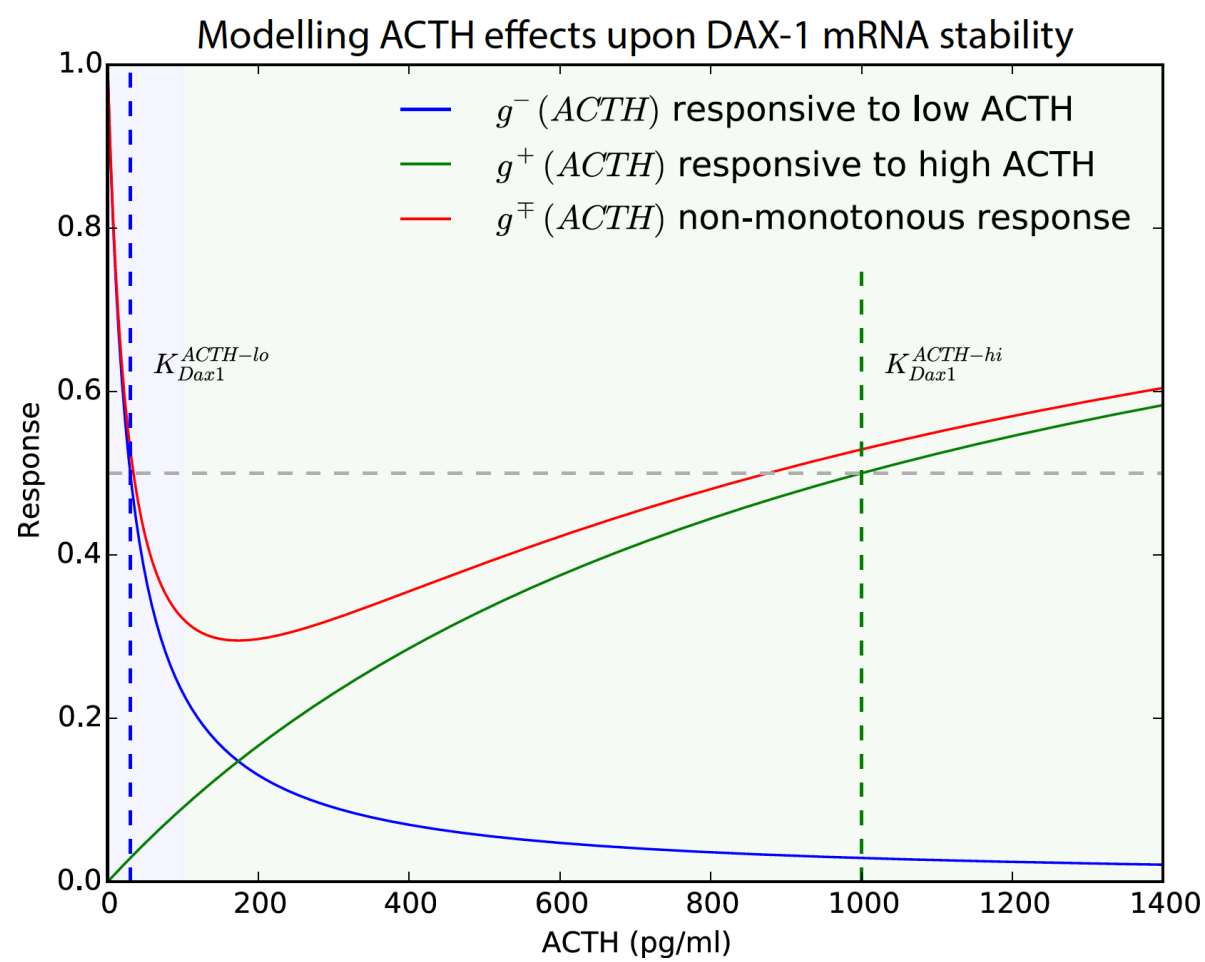

Fig. S4. Modelling ACTH dose-dependent effects on DAX-1 mRNA stability. While an increasing function $\mathrm{g}^{+}$is supported by previous experiments performed at high doses of $\mathrm{ACTH}$, only a decreasing function $\mathrm{g}^{-}$fits our in vivo experiments at low doses of $\mathrm{ACTH}$. We hypothesise a non-monotonous -decreasing, then increasing- response mechanism that successfully reproduces our data at both low and high doses of ACTH. 

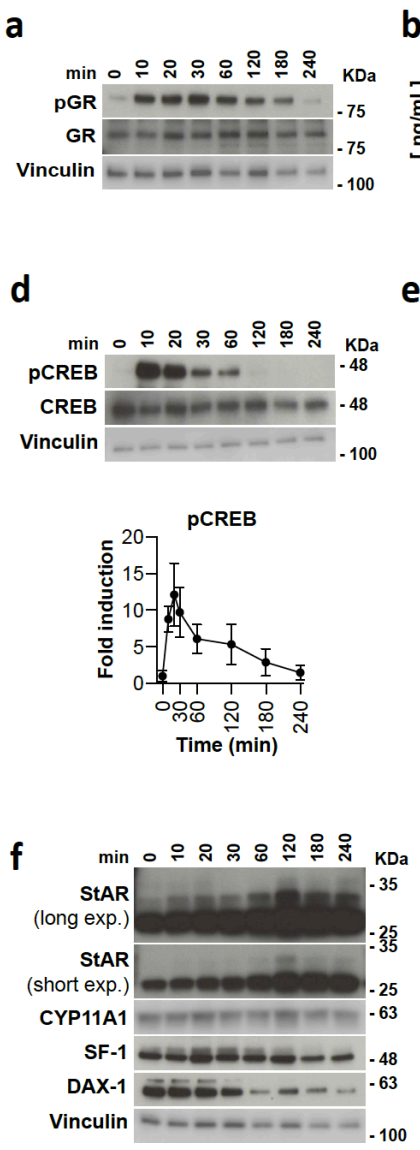

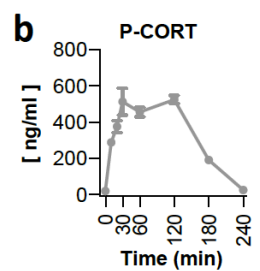

e

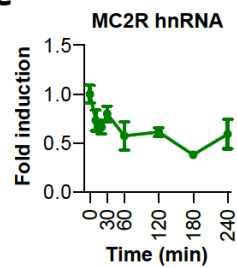

MC2R MRNA

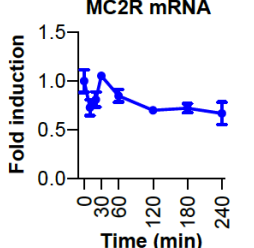

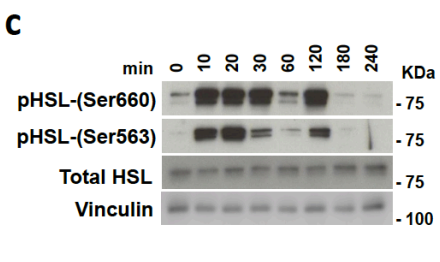
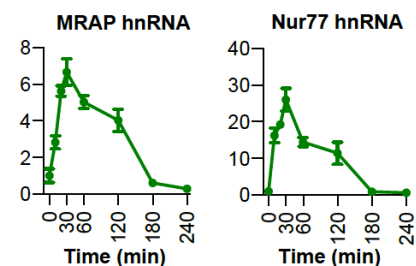

MRAP MRNA

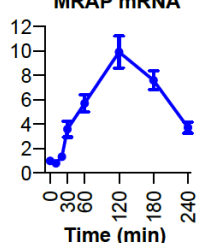

Nur77 mRNA

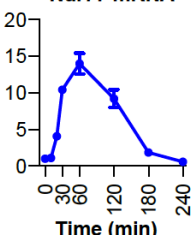

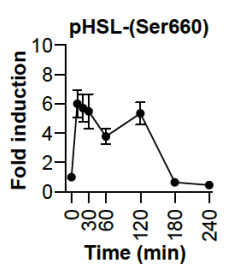
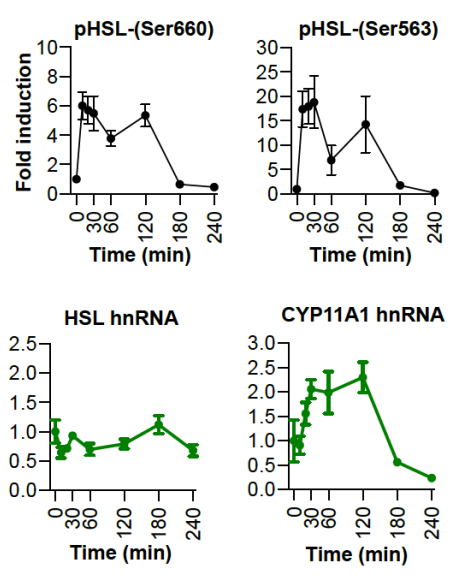

HSL MRNA
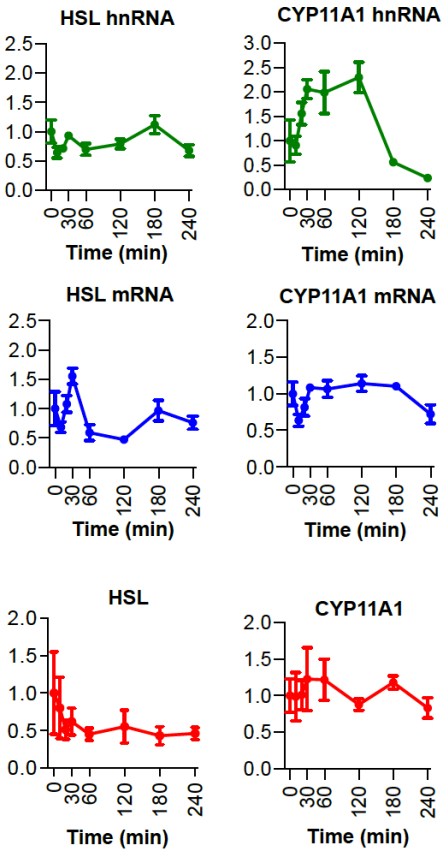

Fig. S5. Dynamic synthesis of adrenal glucocorticoids and activity of the SRN following a high dose of ACTH. (a) Representative Western immunoblotting of the effect of a high dose of ACTH on intra-adrenal phospho-GR (quantification is shown in Fig. $3 \mathrm{c}$ in the main text). (b) A high dose of ACTH dynamically affects plasma CORT (P-CORT). (c) Representative Western immunoblotting and quantification of the dynamic effect of a high dose of ACTH on phosphorylation of HSL (pHSL-(Ser660) and pHSL-(Ser563)). Representative Western immunoblotting and quantification of the dynamic effect of a high dose of ACTH on phosphorylation of CREB in Ser133. (e) A high dose of ACTH dynamically affects plasma CORT steroidogenic genes hnRNA and mRNA. (f) Representative Western immunoblotting and quantification of the dynamic effect of a high dose of ACTH on steroidogenic protein expression (quantification of StARp37, SF-1 and DAX-1 proteins is shown in Fig. 3 in the main text). For Western immunoblotting data, optical density was normalised to Vinculin; for RTqPCR, data were normalised to GAPDH mRNA levels; Western immunoblotting and RTqPCR data are expressed as fold induction of time 0 $(n=4 /$ time point). Details of asymptotic significances are reported in Table S2. 
a

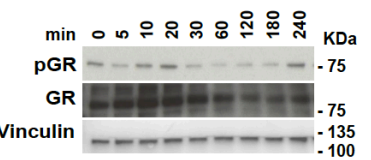

d
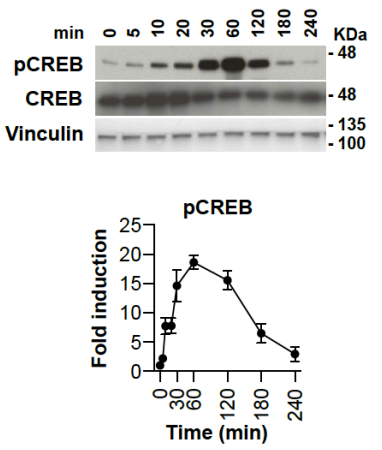

f

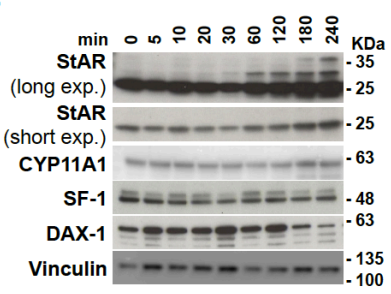

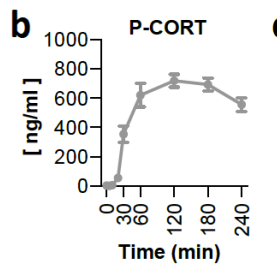

e
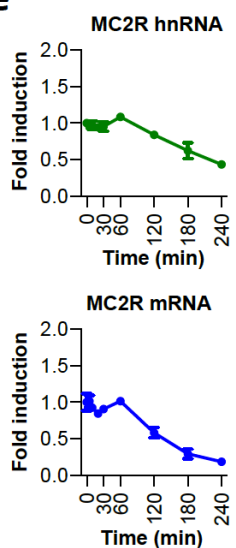

C
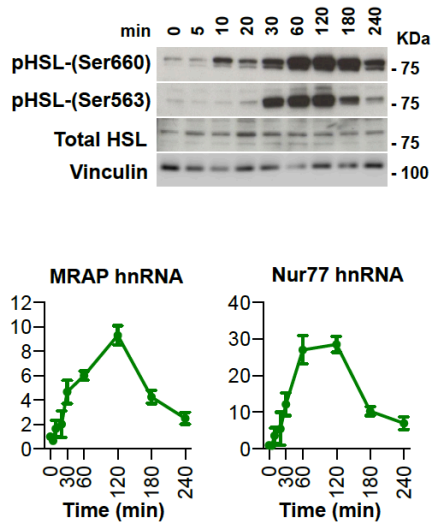

MRAP MRNA
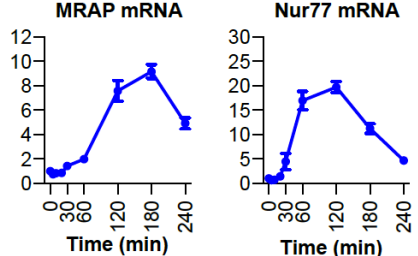
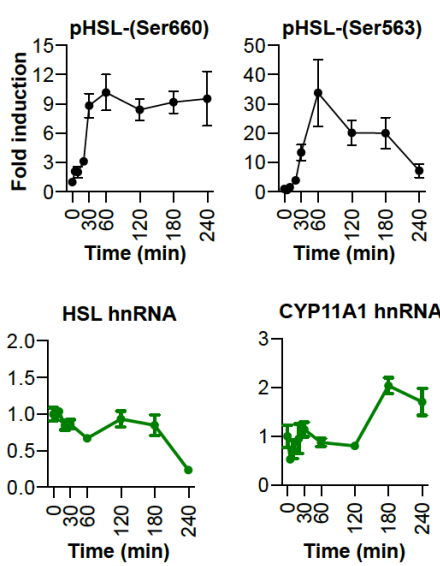

CYP11A1 hnRNA
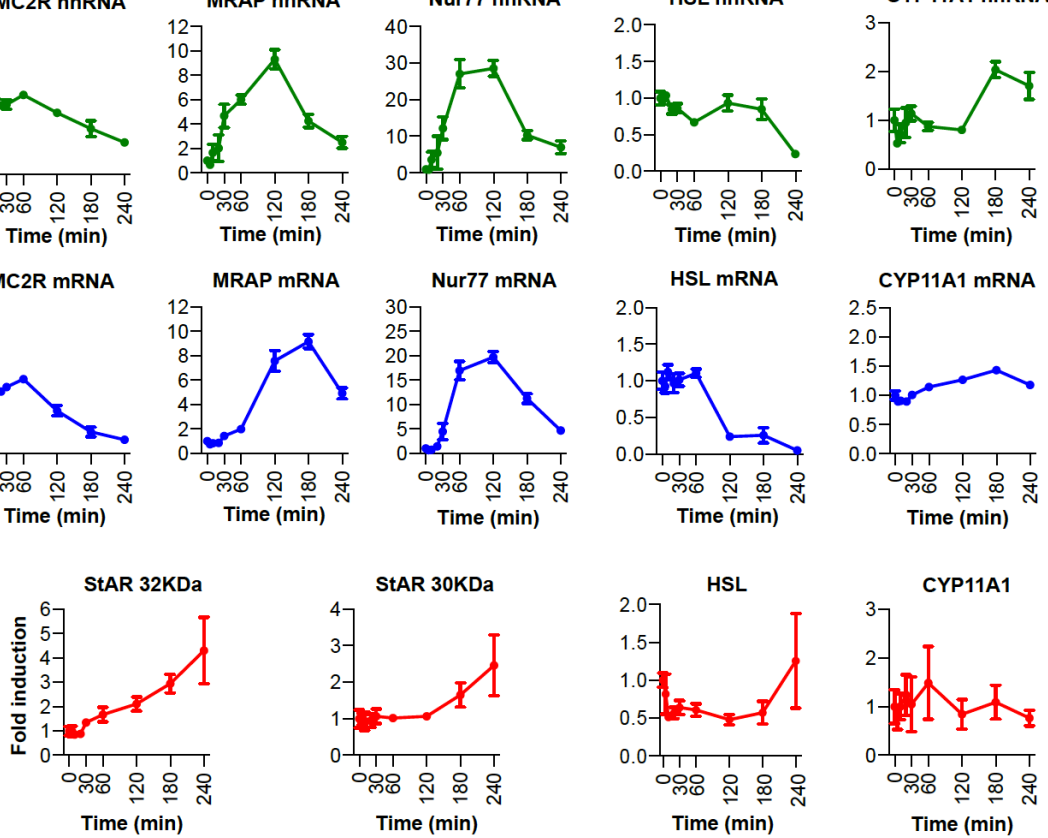

CYP11A1
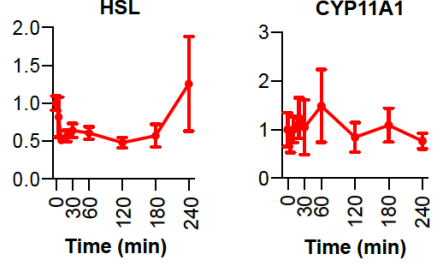

Fig. S6. Dynamic synthesis of adrenal glucocorticoids and activity of the SRN following administration of LPS. (a) Representative Western immunoblotting of the effect of LPS on intra-adrenal phospho-GR (quantification is shown in Fig. 4c in the main text). (b) Administration of LPS dynamically affects plasma CORT (P-CORT). (c) Representative Western immunoblotting and quantification of the dynamic effect of LPS on phosphorylation of HSL (pHSL-(Ser660) and pHSL-(Ser563)). (d) Representative Western immunoblotting and quantification of the dynamic effect of LPS on phosphorylation of CREB in Ser133. (e) Administration of LPS dynamically affects plasma CORT steroidogenic genes hnRNA and mRNA. (f) Representative Western immunoblotting and quantification of the dynamic effect of LPS on steroidogenic protein expression (quantification of StARp37, SF-1 and DAX-1 proteins is shown in Fig. 4 in the main text). For Western immunoblotting data, optical density was normalised to Vinculin; for RTqPCR, data were normalised to GAPDH mRNA levels; Western immunoblotting and RTqPCR data are expressed as fold induction of time 0 ( $n=4-12 /$ time point). Details of asymptotic significances are reported in Table S2. 

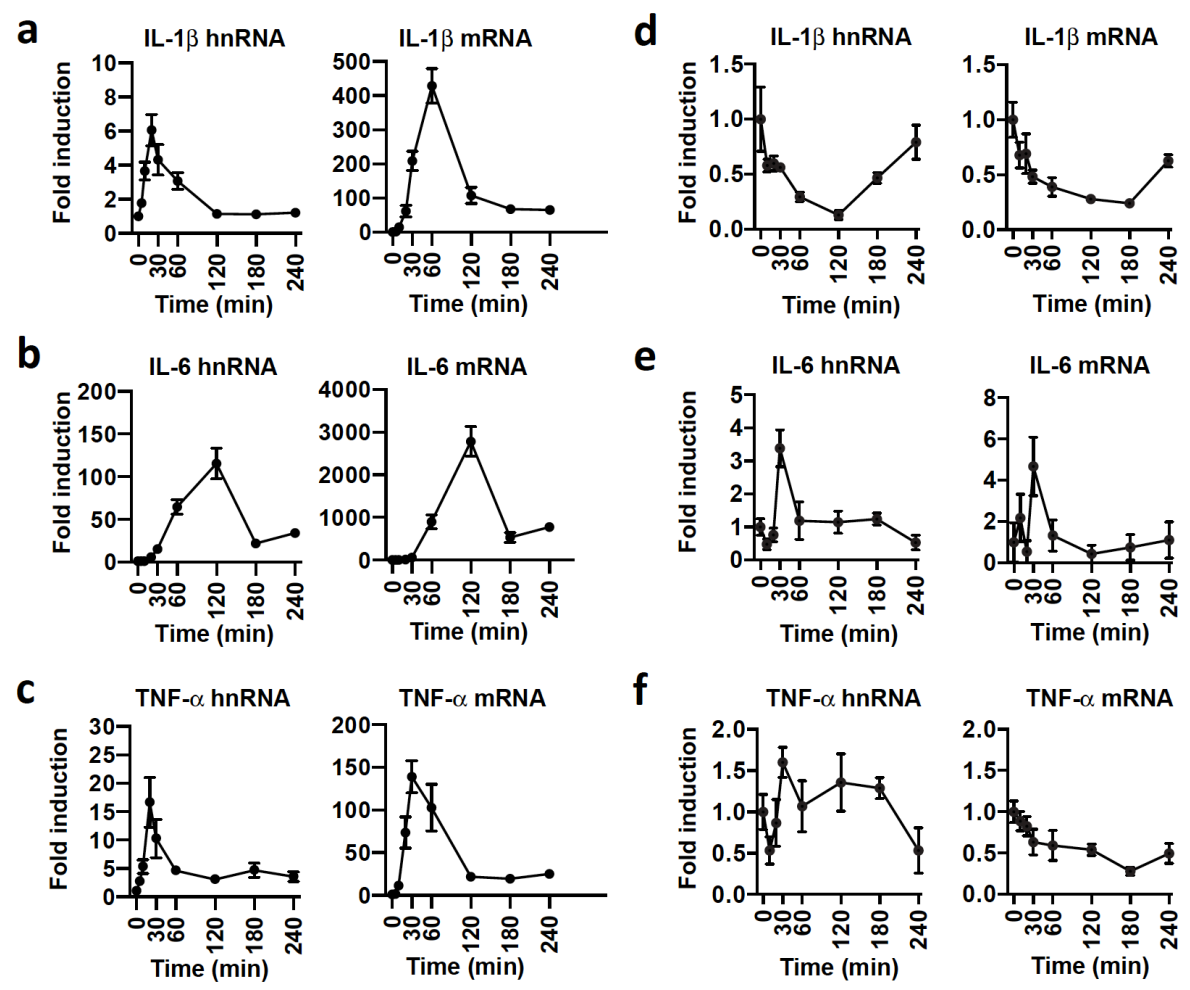

Fig. S7. Effect of LPS vs direct administration of a high dose of ACTH on intra-adrenal cytokines gene expression. The effect of LPS and high s.c. ACTH was evaluated by measuring the hnRNA and mRNA dynamics by RTqPCR ( $n=4-5 /$ time point). Data are expressed as fold induction of time 0. LPS administration dynamically increased (a) IL-1 $\beta$ hnRNA $(P<0.0001)$ and mRNA $(P<0.0001)$, (b) IL-6 hnRNA $(P<0.0001)$ and mRNA $(P<0.0001)$, and (c) TNF- $\alpha$ hnRNA $(P=0.006)$ and mRNA $(P<0.0001)$. In contrast, administration of a high dose of ACTH significant decreased (d) IL-1 $\beta$ hnRNA ( $P=0.005)$ and mRNA ( $P=0.003$ ), whereas only a small but significant increase was observed in (e) IL-6 hnRNA $(P=0.03)$ but not on mRNA $(P=0.171)$, and a trend toward significant increase and a significant decrease was observed in (f) TNF- $\alpha$ hnRNA $(P=0.067)$ and mRNA $(P=0.02)$, respectively. 


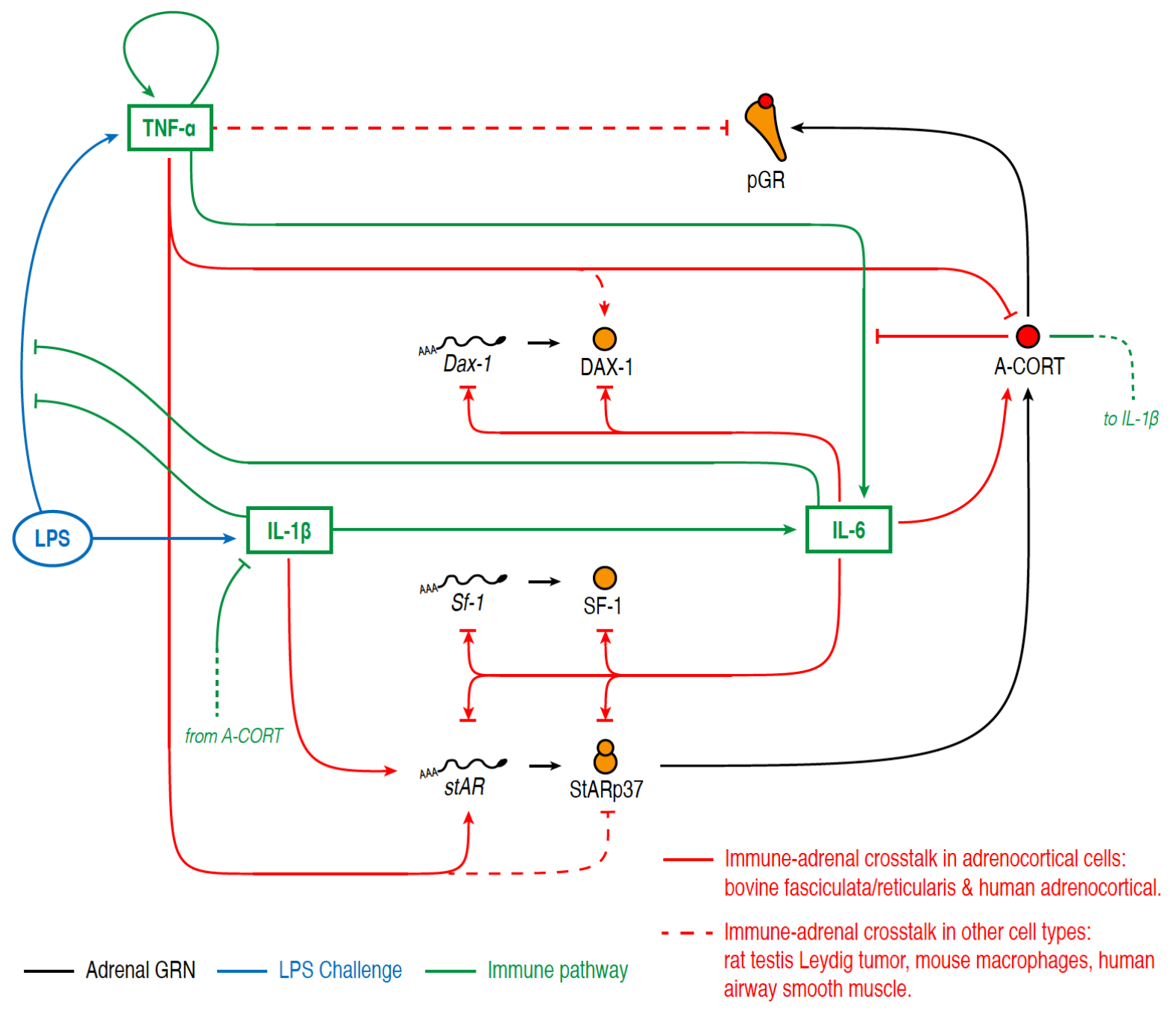

Fig. S8. Cross-talk between the SRN and the immune pathway. During the inflammatory response elicited by LPS, the synthesis of glucocorticoids in adrenocortical cells is modulated by the immune pathway through cytokines. The SRN, in turn, also feeds back upon these cytokines, thus exhibiting cross-talk between both regulatory networks. 

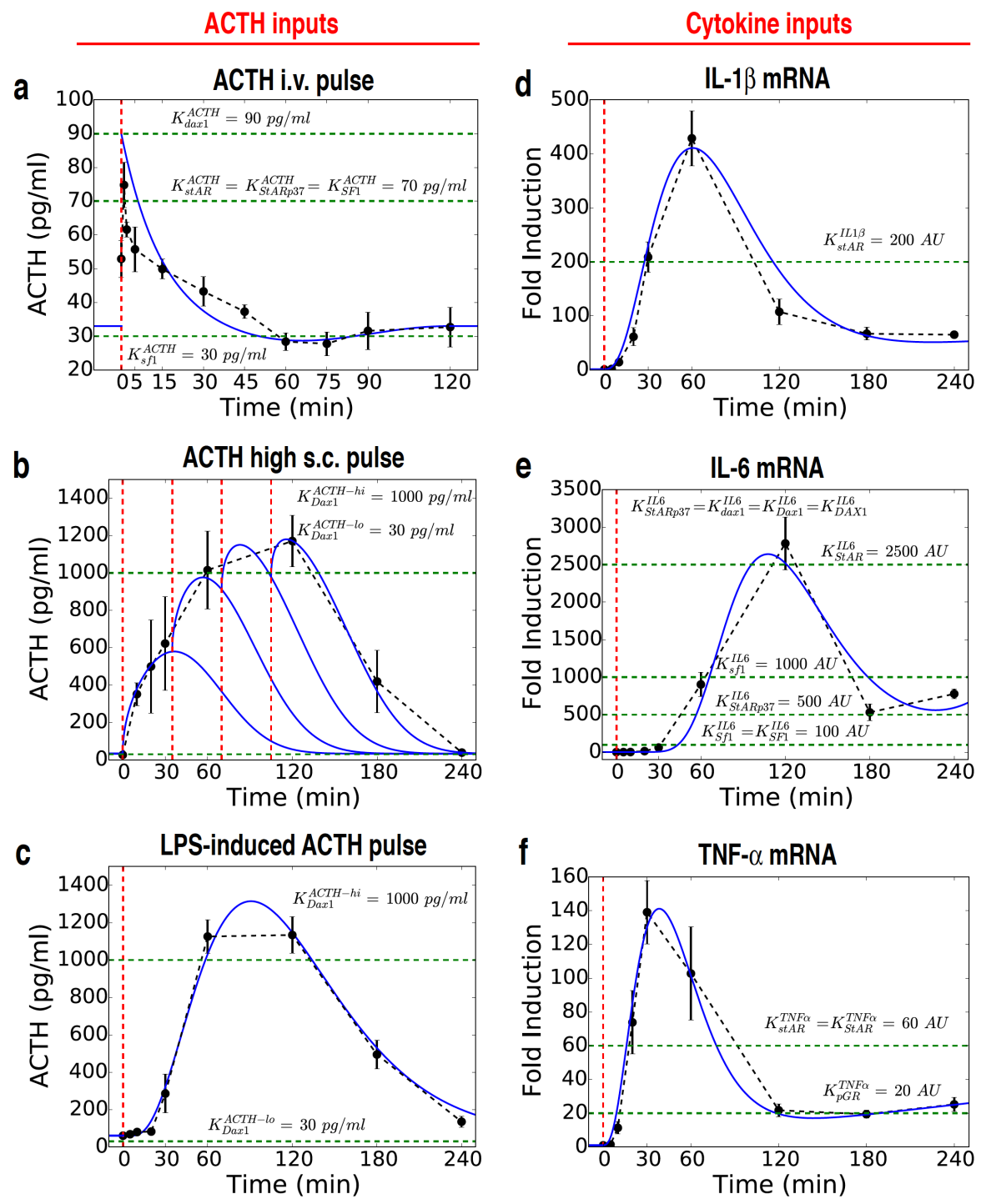

Fig. S9. ACTH and cytokine "virtual" pulse input functions used in the computer simulations and their associated sensitivities. (a) Small pulse (low dose) of ACTH, (b) large pulse of ACTH integrated by four cumulative doses, (c) large pulse of ACTH elicited by LPS, (d-f) cytokine pulses elicited by LPS. 


\section{Supplementary Tables}

Supplementary Table 1. Primer Sequences.

$\begin{array}{lll}\text { Target } & \text { Forward Primer } & \text { Reverse Primer } \\ \text { CYP11A1 hnRNA } & \text { TGTGTGTGTGACCCCAGGAGAC } & \text { CCCAGGTCCTGCTTGAGAGGCT } \\ \text { CYP11A1 mRNA } & \text { TGCGAGGGTCCTAACCCGGA } & \text { ACCTTCCAGCAGGGGCACGA } \\ \text { DAX-1 hnRNA } & \text { TCCAGGCCATCAAGAGTTC } & \text { AAGCTCACCCACTTGACCAC } \\ \text { DAX-1 mRNA } & \text { TCCAGGCCATCAAGAGTTC } & \text { GTGCTCAGTGAGGATCTGC } \\ \text { GAPDH mRNA } & \text { CCATCACTGCCACCCAGAAGA } & \text { GACACATTGGGGGTAGGAACA } \\ \text { HSL hnRNA } & \text { AGGTAGGAGCTGTACCCCTG } & \text { CTGCAAAGACGTTGGACAGC } \\ \text { HSL mRNA } & \text { TATCCGCTCTCCGGTTGA } & \text { CGAGCACTGGAGGAGTGTT } \\ \text { IL-1ß hnRNA } & \text { AGTTGTCCGTGTGTATGGGATG } & \text { GCCAGGCAGAAAGGTTTTGTT } \\ \text { IL-1 } 3 \text { mRNA } & \text { ACCTATGTCTTGCCCGTGGA } & \text { AGGTCGTCATCATCCCACGA } \\ \text { IL-6 hnRNA } & \text { CCCAGAGCACTCCACAAGG } & \text { TCTTGGTCCTTAGCCACTCCT } \\ \text { IL-6 mRNA } & \text { AGCCACTGCCTTCCCTACTT } & \text { GCCATTGCACAACTCTTTTCTCA } \\ \text { MC2R hnRNA } & \text { GAAGTCCGTGAGGTTGSACA } & \text { TTGTGCGGAAGGATCCAGTTT } \\ \text { MC2R mRNA } & \text { GCTTTTGATCCCTGCTTTGAGTG } & \text { CATCTGTTAAAGAAGGAAAGGCTGG } \\ \text { MRAP hnRNA } & \text { ACCTCATTCCTGTGGACGAG } & \text { ACCCGCCATATTATCACTGC } \\ \text { MRAP mRNA } & \text { CCTCCCGGTGTGGGCCTCT } & \text { GGGGACTATGCCTTACCTGTGGGG } \\ \text { NR4A1 hnRNA } & \text { CTTGTGGGGTCCTGCCTGC } & \text { ACGTGGAGAAGGGGCGGTT } \\ \text { NR4A1 mRNA } & \text { GCGGAACCGCTGCCAGTTCT } & \text { GCATCTGGGGGCTGCTTGGG } \\ \text { SF-1 hnRNA } & \text { AGAGGGTGATGGGCTGCT } & \text { ACCTCCACCAGGCACAATAG } \\ \text { SF-1 mRNA } & \text { CGCCAGGAGTTTGTCTGTCT } & \text { ACCTCCACCAGGCACAATAG } \\ \text { StAR hnRNA } & \text { GCAGCAGCAACTGCAGCACTAC } & \text { GTGCCCCCGGAGACTCACCT } \\ \text { StAR mRNA } & \text { CTGGCAGGCATGGCCACACA } & \text { GGCAGCCACCCCTTGAGGTC } \\ \text { TNF- } \alpha \text { hnRNA } & \text { CCGTGACTGTAATCGCCCTAC } & \text { CTTTAGGAGGCTGCAGAGAGAC } \\ \text { TNF- } \alpha \text { mRNA } & \text { AAATGGGCTCCCTCTATCAGTTC } & \text { TCTGCTTGGTGGTTTGCTACGAC }\end{array}$


Supplementary Table 2. Statistical information (P values). Related to Figs. $2-4,6$, and to Figs. S3,5,6.

Component

\begin{tabular}{|c|c|}
\hline ACTH & $<0.0001$ \\
\hline A-CORT & $<0.000$ \\
\hline P-CORT & $<0.000$ \\
\hline pHSL-(Ser660) & $<0.000$ \\
\hline pHSL-(Ser563) & $<0.000$ \\
\hline pCREB & 0.001 \\
\hline StAR hnRNA & $<0.000$ \\
\hline StAR mRNA & 0.067 \\
\hline CYP11A1 hnRNA & $<0.000$ \\
\hline CYP11A1 mRNA & 0.013 \\
\hline MRAP hnRNA & $<0.000$ \\
\hline MRAP mRNA & $<0.000$ \\
\hline MC2R hnRNA & 0.016 \\
\hline MC2R mRNA & $<0.000$ \\
\hline HSL hnRNA & 0.517 \\
\hline HSL mRNA & 0.221 \\
\hline Nur77 hnRNA & $<0.000$ \\
\hline Nur77 mRNA & $<0.000$ \\
\hline SF-1 hnRNA & 0.008 \\
\hline SF-1 mRNA & 0.361 \\
\hline DAX-1 hnRNA & 0.001 \\
\hline DAX-1 mRNA & 0.154 \\
\hline StARp37 & 0.755 \\
\hline StARp32 & 0.967 \\
\hline StARp30 & 0.354 \\
\hline CYP11A1 & 0.482 \\
\hline HSL & 0.777 \\
\hline SF-1 & 0.385 \\
\hline DAX-1 & 0.728 \\
\hline pGR & 0.032 \\
\hline
\end{tabular}

\section{$P$ value}

Fig. 3 and Fig. S5 Figs. 4,6 and Fig. S6

$\begin{array}{cc}0.002 & <0.0001 \\ <0.0001 & <0.0001 \\ <0.0001 & <0.0001 \\ 0.001 & <0.0001 \\ 0.003 & <0.0001 \\ 0.028 & <0.0001 \\ 0.001 & <0.0001 \\ <0.0001 & <0.0001 \\ 0.002 & 0.007 \\ 0.051 & <0.0001 \\ <0.0001 & <0.0001 \\ <0.0001 & <0.0001 \\ 0.051 & 0.002 \\ 0.002 & <0.0001 \\ 0.16 & 0.006 \\ 0.021 & <0.0001 \\ <0.0001 & <0.0001 \\ <0.0001 & <0.0001 \\ 0.003 & 0.005 \\ 0.002 & 0.091 \\ 0.001 & 0.001 \\ 0.002 & <0.0001 \\ 0.441 & <0.0001 \\ 0.457 & <0.0001 \\ 0.007 & 0.433 \\ 0.814 & 0.971 \\ 0.993 & 0.146 \\ 0.492 & 0.76 \\ 0.301 & 0.003 \\ 0.003 & 0.528 \\ & \\ 0.001\end{array}$




\section{Supplementary Materials and Methods}

\section{Animals}

All experiments were conducted on adult male Sprague-Dawley rats (Harlan Laboratories, Inc., Blackthorn, UK) weighting 220-250g at the time of arrival. Animals were given a 1-week acclimatization period prior to the start of the experiments, they were maintained under a $14 \mathrm{~h}$ light, $10 \mathrm{~h}$ dark schedule (lights on at $0500 \mathrm{~h}$ ), and housed four per cage with ad libitum access to food and water. All animal procedures were approved by the University of Bristol Ethical Review Group and were conducted in accordance with Home Office guidelines and the United Kingdom Animals (Scientific Procedures) Act, 1986.

\section{Surgery}

Rats were anaesthetised using isoflurane and an indwelling catheter was inserted in the right jugular vein as previously described (Spiga et al., 2007). In brief, the right jugular vein was exposed, and a silastic-tipped (Merck, Whitehouse, NJ) polythene cannula (Portex, Hythe, UK) was inserted into the vessel to allow substance infusion. Cannula was prefilled with pyrogen-free heparinized $(10 \mathrm{lU} / \mathrm{ml})$ isotonic saline; the free end was exteriorized through a scalp incision and then tunnelled through a protective spring that was anchored to the parietal bones using two stainless steel screws and self-curing dental acrylic. For the high dose ACTH experiment, in addition to the intravenous cannula, during the same surgery a subcutaneous cannula was implanted under the skin between the shoulder blades. After recovery, animals were housed in individual cages in a soundproof room. The end of the protective spring was attached to a mechanical swivel that rotated through $360^{\circ}$ in a horizontal plane and $180^{\circ}$ through a vertical plane, allowing the rats to maximize freedom of movement. The cannula was flushed daily with heparinized saline to maintain patency.

\section{Experiments and tissue collection}

All experiments started at 9 AM and were performed 5-7 days after the surgery. ACTH pulse experiment. To investigate the dynamic adrenal response to an ACTH pulse rats were given an intravenous injection of synthetic ACTH (10 ng per $0.1 \mathrm{ml}$, i.v.; Synachten, Alliance Pharma, Cheltenam, United Kingdom). High dose ACTH experiment. To investigate the effect of a high dose of ACTH, that is able to produce similar plasma ACTH levels as observed after LPSs treatment, rats were given four injections of ACTH $(2 \mu \mathrm{g} / \mathrm{kg}$, sc; Synachten Depot, AlliancePharma, Cheltenam, United Kingdom) at 35-minute intervals, as previously described in (Gibbison et al., 2015). LPS experiment. To investigate the dynamic 
adrenal response to an inflammatory stress rats were given an intravenous injection of LPS (Escherichia coli, clone 055:B5; $25 \mu \mathrm{g} / \mathrm{rat}$ in $0.1 \mathrm{~mL}$ of sterile saline; Sigma, Dorset, United Kingdom).

At the end of each experiment, rats were overdosed with $0.2 \mathrm{~mL}$ of sodium pentobarbitone (Euthatal, $200 \mathrm{mg} / \mathrm{mL}$; Merial, Harlow, United Kingdom) at specific time points shown in the figures. Trunk blood was collected in ice-cold tubes containing EDTA (0.5 M; pH 7.4) and Trasylol (Aprotinin, 500,000 KIU/mL, Roche Diagnostics). Plasma was separated by centrifugation and then stored at $-80^{\circ} \mathrm{C}$ until processed for $\mathrm{ACTH}$ and corticosterone measurement.

Adrenal glands were collected and the inner zones (comprising the zona fasciculata and the zona reticularis of the cortex and the adrenal medulla) were separated from the outer zone (containing the zona glomerulosa and the capsula). Individual inner zones were immediately frozen until processing for isolation of RNA for real-time quantitative polymerase chain reaction (RTqPCR; left adrenal), and for protein extraction for Western immunoblotting and corticosterone measurement (right adrenal) as previously described (Park et al., 2013; Spiga et al., 2011b).

\section{RNA isolation and RT-qPCR}

Total RNA was extracted from the inner zone of individual adrenals using TRIzol reagent (Invitrogen, Hopkinton, MA, USA), followed by purification using RNeasy mini kit reagents, and column DNase digestion (Qiagen, Valencia, CA, USA) to remove genomic DNA contamination. Complementary DNA was reverse transcribed from $1 \mu \mathrm{g}$ of total RNA using Cloned AMV First-Strand cDNA synthesis kit (Invitrogen). Primers were designed to specifically detect primary transcript (hnRNA) or mRNA (Table S1). Fast SYBRGreen Master (Applied Biosystems, Foster City, CA, USA) was used for the amplification mixture with each primer at a final concentration of $200 \mathrm{~nm}$ and $2 \mu \mathrm{l}$ of cDNA for a total reaction volume of $25 \mu \mathrm{l}$. PCR reactions were performed on a spectrofluorometric thermal cycler. The expression of each target gene was normalized to glyceraldehyde 3phosphate dehydrogenase (GAPDH) mRNA as determined in a separate real-time PCR reaction. The absence of RNA detection when the reverse transcription step was omitted indicated the lack of genomic DNA contamination in the RNA samples.

\section{Western immunoblotting}

Whole cell lysate from the inner zone of individual adrenals were prepared using RIPA buffer (Sigma) supplemented with $0.2 \mathrm{mM} \mathrm{Na}$ orthovanadate, $2 \mathrm{mM} \mathrm{NaF}$, and Complete Protease 
Inhibitor (Roche Diagnostics Ltd., Burgess Hill, UK). Protein concentration was quantified by spectrophotometry using the Pierce BCA protein assays, (Thermo Fisher Scientific Inc. Rockford, IL, USA). Aliquots of each sample (10-15 $\mu \mathrm{g}$ of protein) were loaded and separated in a $10 \%$ or $4-15 \%$ Tris-Glycine gel (BioRad, Hercules, CA, USA), transferred to a PVD membrane (GE Amersham Biosciences, Piscataway, NJ, USA), blocked with $5 \%$ non-fat milk or $1 \%$ bovine serum albumin (BSA, sigma) in $1 \times$ Tris-buffered saline plus $0.05 \%$ Tween 20 (TBST) and incubated overnight with Aliquots of each sample (10-15 $\mu \mathrm{g}$ of protein) were loaded and separated in a $10 \%$ or $4-15 \%$ Tris-Glycine gel (BioRad, Hercules, CA, USA), transferred to a PVD membrane (GE Amersham Biosciences, Piscataway, NJ, USA), blocked with $5 \%$ non-fat milk or $1 \%$ bovine serum albumin (BSA, sigma) in $1 \times$ Trisbuffered saline plus $0.05 \%$ Tween 20 (TBST) and incubated overnight with antibodies to StAR, DAX-1, CYP11A1, HSL, GR (Santa Cruz Biotechnologies, Inc., Dallas, TX, US), SF-1 (Upstate Biotechnlogies Inc., Lake Placid, NY, US); CREB, phospho-CREB(Ser133), phospho-HSL(Ser660), phospho-HSL(Ser563); phospho-GR(Ser211) (Cell Signalling Technology, Danvers, MA, US).

After washing with TBST, the membranes were incubated with a horseradish peroxidaseconjugated donkey anti-rabbit IgG or donkey anti-goat IgG (Santa Cruz Biotechnologies). Immunoreactive bands were visualized using ECL Plus TM reagents (GE Amersham Biosciences) followed by exposure to BioMax MR film (Eastman Kodak; Rochester, NY, USA). After film exposure, blots were stripped and assayed for anti-goat vinculin (Santa Cruz Biotechnology). The intensity of the protein targets bands integrated with the area was quantified using a computer image analysis system, Image $\mathrm{J}$ (developed at the National Institutes of Health and freely available at: http://rsb.info.nih.gov). Data points for each gene were then normalized relative to the vinculin band in the respective sample.

\section{Hormone measurement}

Adrenal CORT was measured in adrenal whole cell extract prepared for Western blotting and CORT levels were normalized to the total protein content. Total plasma and adrenal CORT was measured by radioimmunoassay (RIA) using a citrate buffer $(\mathrm{pH} 3.0)$ to denature the binding globulin as previously described (Spiga et al., 2007). Antiserum was kindly supplied by Professor Gabor Makara (Institute of Experimental Medicine, Budapest, Hungary) and [125I] CORT was purchased from Izotop (Budapest, Hungary). ACTH in plasma was measured by RIA using a commercially available assay (MP Biomedicals, Santa Ana, California, USA) in accordance with the manufacturer's instructions. 


\section{Statistics}

Sample sizes in each experiment were determined on the basis of pilot studies and previous experience with similar experimental design. Animals were allocated to each experimental group (time point after treatment) by simple randomization. Experimenters were blinded to the experimental group at the time of hormones, RNA and protein measurements.

Data are represented as the mean \pm SEM, hnRNA, mRNA and protein data are expressed as fold induction of basal (time 0 ). No animals or samples were excluded from statistical analysis. To check data for normality and equality of variance, we used Shapiro-Wilk and Leven tests, respectively. The overall effect of treatments was analysed using the Independent-Samples Kruskal-Wallis Test. Asymptotic significances for each experiment are shown in Table S2. Statistical significance was set at $P<0.05$. 


\section{Mathematical Model}

\section{Contents}

1 Model Development 2

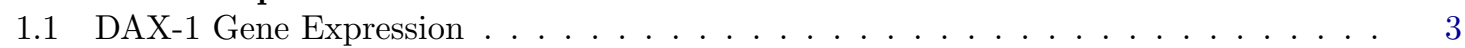

1.2 SF-1 Gene Expression . . . . . . . . . . . . . . . . . . . . . . . . 4

1.3 StAR Gene Expression . . . . . . . . . . . . . . . . . . . . . 5

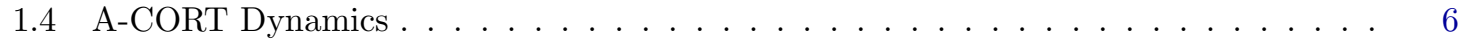

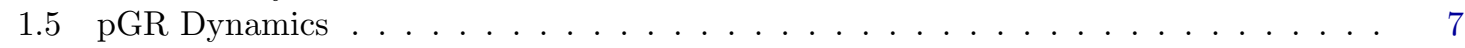

1.6 Model Equations for the SRN . . . . . . . . . . . . . . . . . . . 7

2 Parameter Estimation $r$

2.1 Basal Transcription Rates . . . . . . . . . . . . . . . . . . . . . . . . 9

2.2 Transcription, Translation and Activation Rates _ . . . . . . . . . . . . . . . . . . 9

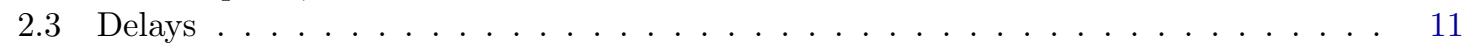

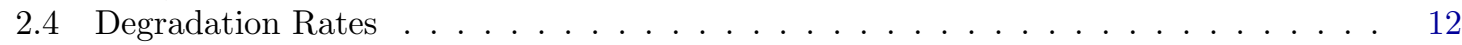

2.5 Half-Maximum Constants . . . . . . . . . . . . . . . . . . . . 14

3 Crosstalk Between the Adrenal SRN and the Immune Pathway 16

3.1 Model Equations for the SRN with Cytokine Interactions . . . . . . . . . . . . 16

3.2 Scaling Model Outputs of Cytokine Targets . . . . . . . . . . . . . . . 18

4 Supplementary References $r$ 


\section{Model Development}

The adrenal SRN controlling the CORT biosynthetic pathway is very complex (Fig. S1). In order to develop a useful yet, manageable mathematical model, we simplified the network by reducing the number of components to a minimum (Fig. 1). Our model predictions support these gene components as the core regulatory clockwork of the SRN. A list of source references to the connections between nodes in both the full and reduced network is shown in Table A.

The mathematical model is representative of a single adrenocortical cell stimulated by an ACTH input. Specifically, ACTH stimulates adrenocortical cells for controlling the biosynthesis of CORT, which, respectively, represent the 'input' and 'output' of the SRN. Since our model is decoupled from other components of the HPA axis, we reproduced endogenous ACTH levels originated from the brain by using an ultradian oscillatory input $(\mathrm{T}=75 \mathrm{~min})$ with circadian modulated amplitude. Thus, in basal, non-stressed conditions, the model dynamics is not static but exhibits an ultradian and circadian rhythmicity driven by the ACTH input. Some model assumptions and biological considerations are the following:

i) The model doesn't focus on the specifics of the CORT biosynthetic pathway within the mitochondria or its release into the bloodstream. Instead, we consider A-CORT as a better readout of the network since its increase occurs earlier than P-CORT, thus reflecting the very first product of CORT biosynthesis.

ii) The network architecture emphasises the crosstalk between StAR, DAX-1 and SF-1 genes. For each of these genes, their hnRNA, mRNA and protein species are modelled explicitly. Though MC2R, MRAP, Nur77 and HSL genes are included in the full network map (Fig. S1), the network architecture suggests they're not directly involved in feedback regulation within the genomic pathway.

iii) Likewise, even though the dynamics of the different phosphorylation states of the HSL protein (pHSL-S565, pHSL-S563 and pHSL-S660) is important to understand cholesterol synthesis, it is StARp37 the one controlling the mitochondrial import of cholesterol, which is the ratelimiting step for ACORT biosynthesis. Accordingly, we didn't include the pHSL dynamics in the present model.

iv) To decouple the intra-adrenal system from the whole HPA axis, we assumed ACTH as an externally controlled input that targets specific components within the adrenal SRN. This took the form of either an endogenous ultradian input with circadian amplitude, a single pulse perturbation, or a combination of both.

Furthermore, we simplified the model by reducing the number of model equations. For instance, cAMP, PKA and pCREB intermediary species in the signalling cascade ending in promoter regulation are not modelled explicitly. Though pCREB is known to regulate transcription of StAR [Manna et al., 2009; Sugawara et al., 1997a,b; Zhao et al., 2005] as well as of other steroidogenic genes, the components of the cascade are known to be downstream of the ACTH pathway, which is already considered in the model.

To reproduce experimental conditions, we administered "virtual" ACTH pulses near the circadian nadir of the endogenous ACTH oscillations. Specifically, we simulated 7 pulses, one every $10 \mathrm{~min}$, distributed between $8 \mathrm{AM}$ and $9 \mathrm{AM}$ of the day. The dynamics during the $120 \mathrm{~min}$ following the stimuli was recorded and averaged for the ACTH i.v. pulse experiments, and during 240 min for the high s.c. ACTH and LPS challenge experiments. 


\subsection{DAX-1 Gene Expression}

The dynamics of gene activation, represented by the newly synthesised, non-edited hnRNA $\operatorname{dax} 1^{1}$, can be represented by the following equation:

$$
\dot{d a x} 1=\sigma_{d a x 1}+k_{d a x 1} f_{d a x 1}(S F 1, p G R) g_{d a x 1}^{-}(A C T H)-\gamma_{d a x 1} d a x 1
$$

where $\sigma_{d a x}$ is the basal transcription rate, $k_{d a x}$ is the maximum transcription rate, $\gamma_{\operatorname{dax} 1}$ is the dax 1 hnRNA degradation rate, and $f_{d a x}$ is a function accounting for dax 1 transcription initiation by the synergistic action of SF1 and pGR, which is modulated by ACTH. Since the specific molecular mechanisms governing the Dax $1^{2}$ promoter activation are still poorly understood, we take an heuristic approach and propose a phenomenological function that captures the effects observed in the study by [Gummow et al., 2006], in which SF1 and GR were cotransfected with a Dax1-Luc reporter into JEG3 cells and stimulated with varying doses of dexamethasone (thus mimicking steroid activation of the GR). Subsequently, a similar experiment was performed in Y1 cells which were concomitantly stimulated with ACTH to assess the level of induction when SF1 and GR were present separately or simultanouesly.

CORT-activated GR, which we measured via its phosphorylated form $p G R$, is known to synergise with SF1 to enhance Dax1 gene expression (up to 30-fold activation in a dose-dependent dexamethasone stimulation experiment). In addition, SF1 is known to also activate the promoter in a pGR-independent way (11-fold activation). Moreover, Glucocorticoid Responsive Elements (GREs) have been identified in the Dax1 promoter and they are dose-responsive to dexamethasone stimulation in the absence of SF1 [Gummow et al., 2006]. However, we will not consider SF1-independent effects on $f_{d a x 1}$ as the elicited fold activation is not only very close to baseline but also small when compared against SF1-pGR synergistic activation and SF1-dependent induction. Further experiments performed by [Gummow et al., 2006] suggest that SF1 and pGR form a molecular complex at the Dax1 promoter which enhances its expression upon steroid stimulation. Considering this and the role that SF1 has in regulating the expression of the StAR gene after binding to the DAX1 protein (thus forming another molecular complex) [Sugawara et al., 1997a,b; Fan et al., 2004; Gummow et al., 2006; Manna et al., 2009], we propose that $f_{\text {dax } 1}$ takes the form:

$$
f_{d a x 1}(S F 1, p G R)=\frac{\frac{S F 1}{K_{S F 1}}+\frac{S F 1 \cdot p G R}{K_{S F 1} \cdot K_{G}}}{1+\frac{S F 1}{K_{S F 1}}+\frac{S F 1 \cdot p G R}{K_{S F 1} \cdot K_{G}}}
$$

In Eq. 2, it can be seen that SF1 is necessary for activating the Dax1 promoter, either independently (i.e. in its free form) or forming a complex with pGR. Thus, pGR can be viewed as an enhancer which acts upon Dax1 promoter through the SF1-pGR complex. The non-linearity observed in Eq. 2 is expected from the pGR-independent SF1 binding to any of its three binding sites within the Dax1 promoter and from the synergy between the central SF1 binding site and the proximal GRE binding site that enhances expression [Gummow et al., 2006]. As the specific mechanisms of regulation are unknown, we chose the simplest empirical representation of the Dax1 promoter regulation by SF1 and pGR transcription factors.

Furthermore, [Gummow et al., 2006] also show that both the SF1 and SF1-pGR synergistic activation of the Dax1 promoter are decreased upon ACTH stimulation. We modelled this by multiplying the second term in Eq. 1 by a decreasing function $g_{\text {dax } 1}^{-}(A C T H)$. As the specific mechanisms and molecular intermediaries of ACTH-dependent modulation are unknown, it is convenient to choose a non-linear Hill type function (see Eq. 3). The $K$ 's in Eqs. 1 and 2 account for half-maximum constants which were determined by fitting the model to data.

$$
g_{\text {dax } 1}^{-}(A C T H)=\frac{K_{d a x 1}^{A C T H^{4}}}{K_{d a x 1}^{A C T H^{4}}+A C T H^{4}}
$$

\footnotetext{
${ }^{1}$ The hyphen in $d a x-1, D a x-1, D A X-1, s f-1, S f-1, S F-1$, and $A-C O R T$ labels was dropped to avoid confusion with a minus sign.

${ }^{2}$ In what follows, the non-italicised nomenclature will be used for gene names unless otherwise specified, whereas the italicised will be reserved for state variables used in the mathematical model (e.g. $\operatorname{dax} 1, \operatorname{Dax} 1$, and $D A X 1$ refer, respectively, to hnRNA, mRNA, and protein concentrations).
} 
The mature transcript dynamics is given by

$$
\operatorname{Dax} 1=k_{D a x 1} d a x 1_{\tau_{D a x 1}}-\gamma_{\operatorname{Dax} 1} \operatorname{Dax} 1 \cdot g_{\operatorname{Dax} 1}^{\mp}(A C T H)
$$

where $k_{\text {Dax } 1}$ stands for the mRNA maturation rate and $\gamma_{\text {Dax } 1}$ is the mRNA degradation rate. RTPCR experiments aimed at detecting hnRNA can't distinguish the transcriptional stage at which the RNA is within the nucleus. However, we know that edition processes such as splicing have finished once mature mRNAs are detected. Hence, we assumed Eq. 4 is delayed by $\tau_{\text {Dax } 1}$, which accounts for the elapsed time since transcription initiation up to the emergence of a hnRNA plus the maturation time after completion of transcription (splicing, capping, polyadenylation and mRNA nuclear export processes).

In addition, high doses of ACTH are known to destabilise Dax1 transcripts [Ragazzon et al., 2006], which in a first instance was modelled by multiplying the turnover term in Eq. 4 by a positively increasing function of $\mathrm{ACTH}\left(g_{\operatorname{Dax} 1}^{+}(A C T H)\right)$. However, when re-calibrating the model by fitting it to our ACTH i.v. pulse experiments, we observed a mismatch between its predictions and our DAX1 mRNA and protein data. We realised that our assumption of a monotonously-increasing function was based on experimental results by [Ragazzon et al., 2006] that were performed using very high levels of ACTH. Thus, to fit our model to data from low ACTH i.v. pulse experiments, but keeping consistency with previous observations by [Ragazzon et al., 2006] at high ACTH levels, we hypothesised an ACTH dose-dependent control mechanism of Dax1 mRNA stability that was modelled assuming its turnover rate is modulated by the function $g_{\text {Dax } 1}^{\mp}(A C T H)$. Though it's not clear how ACTH actually promotes or inhibits degradation of Dax1, we propose that a nonmonotonous regulatory function would provide the best fit of the model to our experimental data in both the low and high ACTH stimuli experiments. We model this through a decreasing-thenincreasing function containing two half-maximum constants: $K_{\text {Dax } 1}^{A C T H-l o}$ for inhibition at low doses of ACTH, and $K_{\operatorname{Dax} 1}^{A C T H-h i}$ for activation at high doses of ACTH (Fig. S4). This function takes the form:

$$
g_{D a x 1}^{\mp}(A C T H)=\frac{K_{D a x 1}^{A C T H-l o}}{K_{D a x 1}^{A C T H-l o}+A C T H}+\frac{A C T H}{K_{D a x 1}^{A C T H-h i}+A C T H}
$$

Finally, the DAX1 protein dynamics is governed by:

$$
D \dot{A X} 1=k_{D A X 1} \operatorname{Dax} 1_{\tau_{D A X 1}}-\gamma_{D A X 1} D A X 1
$$

where $k_{D A X 1}$ stands for the protein translation rate, $\tau_{D A X 1}$ is the delay associated to translation, and $\gamma_{D A X 1}$ is the protein degradation rate.

\section{$1.2 \quad$ SF-1 Gene Expression}

The dynamics of gene activation, represented by the newly synthesised, non-edited $s f 1$ hnRNA, can be represented by the following equation:

$$
s \dot{f} 1=\sigma_{s f 1}+k_{s f 1} g_{s f 1}^{+}(A C T H)-\gamma_{s f 1} s f 1
$$

where $\sigma_{s f 1}$ is the basal transcription rate, $k_{s f 1}$ is the maximum transcription initiation rate, and $\gamma_{s f 1}$ is the $s f 1$ degradation rate. To the best of our knowledge, the SF1 gene is not subject to transcriptional regulation by any of the proteins considered in the model. Thus, conversely to Eq. 1, here we have no function accounting for $s f 1$ transcription modulation by any transcription factors other than the effects induced by ACTH. This is modelled by a function $g_{s f 1}^{+}(A C T H)$, which takes the form:

$$
g_{s f 1}^{+}(A C T H)=\frac{A C T H^{4}}{K_{s f 1}^{A C T H^{4}}+A C T H^{4}}
$$

Though in our model we consider that SF1 gene expression is regulated by ACTH alone [Ragazzon et al., 2006], regulation by other transcription factors within the steroidogenic pathway cannot be ruled out. This poses interesting questions about the role of this gene in controlling the adrenal 
response. Moreover, evidence suggests that its mRNA and protein levels remain constant after variations in cAMP concentration, which is a known mediator of the ACTH pathway [Fan et al., 2004].

The mature transcript dynamics is given by:

$$
\dot{S f 1}=k_{S f 1} s f 1_{\tau_{S f 1}}-\gamma_{S f 1} g_{S f 1}(S f 1)
$$

where $k_{S f 1}$ stands for the mRNA maturation rate and $\gamma_{S f 1}$ is the mRNA degradation rate. By similar arguments as in Eq. 4, we assumed Eq. 9 is delayed by $\tau_{S f 1}$. Importantly, we found out that the best fit of the model to our data was achieved when assuming a Michaelis-Menten degradation of $S f 1$ mRNA. Thus, the function $h_{S f 1}(S f 1)$ takes the form:

$$
h_{S f 1}(S f 1)=\frac{S f 1}{K_{S f 1}+S f 1}
$$

Finally, the $S F 1$ protein dynamics is governed by:

$$
S \dot{F} 1=k_{S F 1} S f 1_{\tau_{S F 1}}-\gamma_{S F 1} S F 1 \cdot g_{S F 1}^{-}(A C T H)
$$

where $k_{S F 1}$ stands for the protein translation rate, $\tau_{S F 1}$ is the delay associated to translation, and $\gamma_{S F 1}$ is the protein degradation rate. Since ACTH is suspected to stabilise $S F 1$, probably through phosphorylation or ubiquitination of the proteasome [Æsøy et al., 2002], we assume its turnover rate is modulated by a function $g_{S F 1}^{-}(A C T H)$, which takes the form:

$$
g_{S F 1}^{-}(A C T H)=\frac{K_{S F 1}^{A C T H}}{K_{S F 1}^{A C T H}+A C T H}
$$

\subsection{StAR Gene Expression}

The dynamics of StAR gene activation, represented by the newly synthesised, non-edited st $A R$ hnRNA, can be represented by the equation:

$$
s t \dot{A} R=\sigma_{s t A R}+k_{s t A R} f_{s t A R}(S F 1, D A X 1) g_{s t A R}^{+}(A C T H)-\gamma_{s t A R} s t A R
$$

where $\sigma_{s t A R}$ is the basal transcription rate, $k_{s t A R}$ is the maximum transcription initiation rate, $\gamma_{s t A R}$ is the $s t A R$ degradation rate, and $f_{s t A R}$ is a function accounting for st $A R$ transcription initiation controlled positively by $S F 1$ and negatively by $D A X 1$. This $D A X 1$-mediated steroidogenic inhibition follows from the finding that $D A X 1$ inhibits $S F 1$ transactivation upon binding it [Babu et al., 2002; Fan et al., 2004], thus preventing StAR gene activation. In addition, ACTH is known to control the StAR gene activation directly through pCREB [Sugawara et al., 1997a,b; Manna et al., 2009]. However, since we're not modelling pCREB explicitly, we employ the function $g_{\text {st } A R}^{+}(A C T H)$ to model the StAR gene activation by ACTH. This function takes the form:

$$
g_{s t A R}^{+}(A C T H)=\frac{A C T H^{4}}{K_{s t A R}^{A C T H^{4}}+A C T H^{4}}
$$

In summary, an $S F 1$ dependent activation mechanism, similar to the one modelled in Eq. 2, has been shown to be responsible for StAR gene activation [Sugawara et al., 1997b; Manna et al., 2003; Fan et al., 2004; Xu et al., 2009]. This follows from cotransfection experiments carried out by [Fan et al., 2004] to explore the effects of $S F 1$ (a.k.a. Ad4BP) and $D A X 1$ concomitantly with Forskolin stimulation of the PKA pathway.

These considerations were taken into account to assume that $f_{\text {stAR }}$ takes the form:

$$
f_{s t A R}(S F 1, D A X 1)=\frac{\frac{S F 1}{K_{S F 1}}}{1+\frac{S F 1}{K_{S F 1}}+\frac{D A X 1}{K_{D}}}
$$

In Eq. 13, it can be seen that both ACTH and $S F 1$ are necessary for activating st $A R$, whereas $D A X 1$ modulates the promoter activity by binding to $S F 1$ at the promoter site (the gene is inactive 
if either ACTH or $S F 1$ are absent, but not if $D A X 1$ is). Importantly, this empirical approach facilitates modifying Eq. 15 if we wish to account for $D A X 1$ dosage dependent effects observed in diseased states [Xu et al., 2009; Sadasivam et al., 2015]. Just as in Eq. 2, the K's in Eq. 15 account for half-maximum constants (often interpreted as sensitivities) and were determined by fitting the model to data.

The mature transcript dynamics is given by:

$$
S t \dot{A} R=k_{S t A R} s t A R_{\tau_{S t A R}}-\gamma_{S t A R} h_{S t A R}(S t A R)
$$

where $k_{S t A R}$ stands for the mRNA maturation rate and $\gamma_{S t A R}$ is the mRNA degradation rate. By similar arguments as in Eq. 4, we assumed Eq. 16 is delayed by $\tau_{S t A R}$. Importantly, we found out that the best fit of the model to data was achieved when assuming a Michaelis-Menten degradation of $S t A R$ mRNA. Thus, the function $h_{S t A R}(S t A R)$ takes the form:

$$
h_{S t A R}(S t A R)=\frac{S t A R}{K_{S t A R}+S t A R}
$$

Finally, for the StAR protein dynamics we will account for the $37 \mathrm{kDa}$ precursor, as it has been shown to be the one responsible for cholesterol import before being itself cleaved and imported to the mitochondria [Arakane et al., 1998; Bose et al., 2002; Manna et al., 2009]. It's dynamics is given by:

$$
S t A \dot{R} p 37=k_{S t A R p 37} S t A R_{\tau_{S t A R p 37}}-\mu_{S t A R p 37} S t A R p 37 g_{\text {StARp } 37}^{-}(A C T H)
$$

where $k_{S t A R p 37}$ stands for the protein translation rate, $\tau_{S t A R p 37}$ is the delay associated to translation, and $\mu_{S t A R p 37}=\gamma_{S t A R p 37}+\epsilon_{S t A R p 37}$ is the StARp37 turnover rate. This value accounts for the proteasome-mediated degradation rate of the active precursor $S t A R p 37, \gamma_{S t A R p 37}$, and the import rate of the StARp37 cleaved byproducts into mitochondria, $\epsilon_{S t A R p 37}$ [Arakane et al., 1997; Bose et al., 2002; Manna et al., 2009]. As it has been show that StARp37 activity is proportional to its residence time in the cytosol [Bose et al., 2002; Granot et al., 2003], and that PKA-mediated phosphorylation stabilises the precursor [Clark and Hudson, 2015], we have included the function $g_{S t A R p 37}(A C T H)$ in the turnover term in Eq. 18, which takes the form:

$$
g_{\text {StARp } 37}^{-}(A C T H)=\frac{K_{\text {StARp } 37}^{A C T H}{ }^{4}}{K_{\text {StARp } 37}^{A C T H}+A C T H^{4}}
$$

\subsection{A-CORT Dynamics}

As mentioned before, the CORT biosynthetic pathway within the mitochondria won't be modelled explicitly at this stage. Instead, we assume that ACORT dynamics is governed by:

$$
A C \dot{O} R T=k_{A C O R T} f_{A C O R T}(S t A R p 37)-\mu_{A C O R T} A C O R T
$$

where $k_{A C O R T}$ is the maximum synthesis rate of ACORT, and the turnover term $\mu_{A C O R T}=$ $\gamma_{A C O R T}+\epsilon_{A C O R T}$ accounts for the ACORT degradation rate $\gamma_{A C O R T}$ and the export rate from the adrenal cell into the bloodstream $\epsilon_{A C O R T}$. The function $f_{A C O R T}$ expresses the dependence of ACORT synthesis on the precursor StARp37 and takes the form:

$$
f_{A C O R T}(S t A R p 37)=\frac{S t A R p 37}{K_{S t A R p 37}+S t A R p 37}
$$

where the big $K$ has the same meaning as in previous functions and was determined by fitting the model to data. As mentioned before, StARp37 controls the import of cholesterol into the mitochondria [Bose et al., 2002], which is the rate limiting step for corticosteroid biosynthesis. We assumed this process can be represented as a Michaelis-Menten reaction (see Eq. 21) [Arakane et al., 1997; Manna et al., 2009; Spiga et al., 2014]. Importantly, although StARp37 is downstream of ACTH (through regulation of the StAR gene), ACORT is known to respond to ACTH stimulation on a much shorter timescale than the time needed for ACTH to exert its effects on StARp37 through the cAMP/PKA/pCREB pathway. This likely happens due to PKA mediated StARp37 stabilisation in the cytosol upon ACTH stimulation [Arakane et al., 1997; Manna et al., 2009], a process that is already accounted for in the turnover term in Eq. 18. 


\section{5 pGR Dynamics}

Lastly, instead of modelling the concentration of the glucocorticoid receptor, we model the measured levels of its phosphorylated form $p G R$, a marker of its activity. We propose the dynamics of the $p G R$ is governed by:

$$
p \dot{G} R=k_{G R} f_{p G R}(A C O R T)-\gamma_{p G R} p G R
$$

where $k_{p G R}$ stands for the $p G R$ activation rate by ACORT and $\gamma_{p G R}$ is it's deactivation rate. The function $f_{p G R}$ takes the form:

$$
f_{p G R}(A C O R T)=\frac{A C O R T}{K_{A C O R T}+A C O R T}
$$

where $K_{A C O R T}$ stands for the half-maximum constant of $p G R$ activation by ACORT.

\subsection{Model Equations for the SRN}

The complete set of model equations is shown below:

$$
\begin{aligned}
d \dot{a x} 1 & =\sigma_{d a x 1}+k_{d a x 1} f_{d a x 1}(S F 1, p G R) g_{\text {dax } 1}^{-}(A C T H)-\gamma_{d a x 1} d a x 1, \\
D \dot{D a x} 1 & =k_{D a x 1} d a x 1_{\tau_{D a x 1}}-\gamma_{D a x 1} \operatorname{Dax} 1 g_{D a x 1}^{\mp}(A C T H), \\
D \dot{A X} 1 & =k_{D A X 1} \operatorname{Dax} 1_{\tau_{D A X 1}}-\gamma_{D A X 1} D A X 1, \\
s \dot{f} 1 & =\sigma_{s f 1}+k_{s f 1} g_{s f 1}^{+}(A C T H)-\gamma_{s f 1} s f 1, \\
S \dot{f} 1 & =k_{S f 1} s f 1_{\tau_{S f 1}}-\gamma_{S f 1} h_{S f 1}(S f 1), \\
S \dot{F} 1 & =k_{S F 1} S f 1_{\tau_{S F 1}}-\gamma_{S F 1} S F 1 g_{S F 1}^{-}(A C T H), \\
s t \dot{A} R & =\sigma_{s t A R}+k_{s t A R} f_{s t A R}(S F 1, D A X 1) g_{s t A R}^{+}(A C T H)-\gamma_{s t A R} s t A R, \\
S t \dot{A} R & =k_{S t A R} s t A R_{\tau_{S t A R}}-\gamma_{S t A R} h_{S t A R}(S t A R), \\
S t A \dot{R} p 37 & =k_{S t A R p 37} S t A R_{\tau_{S t A R p 37}}-\mu_{S t A R p 37} S t A R p 37 g_{S t A R p 37}^{-}(A C T H), \\
A C \dot{O} R T & =k_{A C O R T} f_{A C O R T}(S t A R p 37)-\mu_{A C O R T} A C O R T, \\
p \dot{G} R & =k_{G R} f_{p G R}(A C O R T)-\gamma_{p G R} p G R .
\end{aligned}
$$

where:

$$
\begin{aligned}
& g_{\text {dax } 1}^{-}(A C T H)=\frac{K_{\operatorname{dax} 1}^{A C T H^{4}}}{K_{\text {dax } 1}^{A C T H^{4}}+A C T H^{4}}, \\
& g_{\text {Dax } 1}^{\mp}(A C T H)=\frac{K_{D a x 1}^{A C T H-l o}}{K_{D a x 1}^{A C T H-l o}+A C T H}+\frac{A C T H}{K_{D a x 1}^{A C T H-h i}+A C T H}, \\
& g_{s f 1}^{+}(A C T H)=\frac{A C T H^{4}}{K_{s f 1}^{A C T H^{4}}+A C T H^{4}}, \quad g_{S F 1}^{-}(A C T H)=\frac{K_{S F 1}^{A C T H}}{K_{S F 1}^{A C T H}+A C T H}, \\
& g_{\text {stAR }}^{+}(A C T H)=\frac{A C T H^{4}}{K_{\text {stAR }}^{A C T H^{4}}+A C T H^{4}}, \quad g_{\text {StARp } 37}^{-}(A C T H)=\frac{K_{\text {StARp } 37}^{A C T H}{ }^{4}}{K_{\text {StARp } 37}^{A C T H}+A C T H^{4}}, \\
& h_{S f 1}(S f 1)=\frac{S f 1}{K_{S f 1}+S f 1}, \quad \quad h_{S t A R}(S t A R)=\frac{S t A R}{K_{S t A R}+S t A R}, \\
& f_{A C O R T}(S t A R p 37)=\frac{S t A R p 37}{K_{S t A R p 37}+S t A R p 37}, \quad f_{p G R}(A C O R T)=\frac{A C O R T}{K_{A C O R T}+A C O R T}, \\
& f_{d a x 1}(S F 1, p G R)=\frac{\frac{S F 1}{K_{S F 1}}+\frac{S F 1 \cdot p G R}{K_{S F 1} \cdot K_{G}}}{1+\frac{S F 1}{K_{S F 1}}+\frac{S F 1 \cdot p G R}{K_{S F 1} \cdot K_{G}}}, \quad f_{s t A R}(S F 1, D A X 1)=\frac{\frac{S F 1}{K_{S F 1}}}{1+\frac{S F 1}{K_{S F 1}}+\frac{D A X 1}{K_{D}}} .
\end{aligned}
$$




\begin{tabular}{|c|c|c|c|c|}
\hline Node & Target & Sign & Effect & References \\
\hline \multirow[t]{14}{*}{$\mathrm{ACTH}$} & $\operatorname{dax} 1$ & - & Inhibits SF1-dependent activation & [Gummow et al., 2006] \\
\hline & $\operatorname{Dax} 1$ & $\mp$ & Modulates mRNA stability & [Ragazzon et al., 2006] \\
\hline & $s f 1$ & + & Promoter activation & [Ragazzon et al., 2006] \\
\hline & $S F 1$ & - & Protein stabilisation & [Æsøy et al., 2002] \\
\hline & $s t A R$ & + & pCREB-mediated promoter activation & [Sugawara et al., 1997a] \\
\hline & & & & [Sugawara et al., 1997b] \\
\hline & & & & [Zhao et al., 2005] \\
\hline & & & & [Manna et al., 2009] \\
\hline & StARp37 & + & PKA-mediated protein stabilisation & [Arakane et al., 1997] \\
\hline & & & & [Manna et al., 2009] \\
\hline & HSL & + & PKA-mediated activation (S563 and S660) & [Manna et al., 2013] \\
\hline & & + & AMPK-mediated activation (S565) & [Watt et al., 2006] \\
\hline & $m c 2 r$ & + & Promoter activation & [Winnay and Hammer, 2006] \\
\hline & mrap & + & Promoter activation & [Gorrigan et al., 2011] \\
\hline ACORT & pGR & + & Activation through phosphorylation & --- \\
\hline pGR & $\operatorname{dax} 1$ & + & SF1-dependent enhancement of Dax1 & [Gummow et al., 2006] \\
\hline \multirow[t]{7}{*}{$D A X 1$} & stAR & $+/-$ & Inhibits SF1-dependent activation & [Sugawara et al., 1997a] \\
\hline & & & & [Sugawara et al., 1997b] \\
\hline & & & & [Fan et al., 2004] \\
\hline & & & Activation at high doses & [Xu et al., 2009] \\
\hline & $m c 2 r$ & + & Promoter activation & [Xu et al., 2009] \\
\hline & nur77 & - & Promoter inhibition & [Song et al., 2004] \\
\hline & NUR77 & - & Protein inhibition & [Song et al., 2004] \\
\hline \multirow[t]{4}{*}{$S F 1$} & $\operatorname{dax} 1$ & + & Promoter activation & [Gummow et al., 2006] \\
\hline & $s t A R / m c 2 r$ & + & Promoter activation & [Babu et al., 2002] \\
\hline & & & & [Gummow et al., 2006] \\
\hline & & & & [Winnay and Hammer, 2006] \\
\hline \multirow[t]{3}{*}{ StARp37 } & StARp30/Chol & + & Cholesterol import to mitochondria & [Arakane et al., 1997] \\
\hline & & & & [Manna et al., 2009] \\
\hline & HSL-S660/Chol & + & Cholesterol biosynthesis & [Shen et al., 2003] \\
\hline HSL & $\mathrm{S} 565 / \mathrm{S} 563 / \mathrm{S} 660$ & $+/-$ & Context-dependent phosphorylation & $\begin{array}{c}\text { [Kraemer and Shen, 2002] } \\
\text { [Watt et al., 2006] }\end{array}$ \\
\hline
\end{tabular}

Note: Obvious connections (e.g. transcription, translation, ACORT biosynthesis) are not included in this table.

Table A: Supporting references for cross-talk connections within the adrenal SRN. 


\section{Parameter Estimation}

The temporal resolution of our experiments is determined by the timescale of the regulatory processes involved, which was in the order of minutes. We set this as the timescale for our kinetic parameters, so that all parametric units were expressed in terms of molar concentrations (M) and minutes (min). Moreover, even though our model equations are considering a single cell system, we must not forget that our experimental measurements were carried out at the tissue level. This means our model predictions could be extrapolated to the total number of cells in the adrenal cortex, provided that the appropriate cellular and nuclear volumes for the different adrenal cell types are accounted for. Though we couldn't find measurements of these volumes in Sprague Dawley rats, we approximated them from a study of the ultrastructure of Wistar rat adrenal cells, which estimates the cell and nuclear volume of steroidogenic ZF cells as $\sim 3600 \mu \mathrm{m}^{3}$ and $\sim 260 \mu \mathrm{m}^{3}$, respectively [Nickerson, 1976]. Thus, to deal with Molar units in a simpler way, we used the nuclear volume $V_{n u c}=2.6 \times 10^{-13} l t$ as it is in this cellular enclosure where gene regulatory reactions take place.

Also, note that the regulatory functions have either sigmoidal or Michaelian shapes and that they're normalised (including the multivariate functions). Hence, the overall flux contribution of these terms is to modulate the weight of kinetic rates (small $k$ 's), whereas their sensitivity to activation by the independent variable is represented by the half-maximum constants (big $K$ 's), i.e. the concentration at which the $i$-th factor reaches $\frac{1}{2}$.

In what follows, we estimate the kinetic rates and other parameter values. A comprehensive summary of these values is shown in Table B.

\subsection{Basal Transcription Rates}

The basal transcription rates, denoted by the $\sigma$ 's, are the rates at which the gene promoters are activated without the influence of any of the above-mentioned transcription regulators (thought other factors may be involved), or the minimum level of transcription that these regulators may exert on the gene (see for instance the influx term in Eq. 7 when ACTH is absent). For the sake of simplicity, we assumed that basal transcription rates amounted to $5 \%$ of the maximum transcription rates. That is:

$$
\sigma_{d a x 1}=0.05 * k_{d a x 1}, \quad \sigma_{s f 1}=0.05 * k_{s f 1}, \quad \sigma_{s t A R}=0.05 * k_{s t A R} .
$$

\subsection{Transcription, Translation and Activation Rates}

We calculated the maximum rate of gene expression, assessed by the synthesis of the nascent transcript, as:

$$
k_{\text {gene }}=\frac{k_{\text {pol }}^{\max }}{L_{h n R N A} N_{A} V_{\text {nuc }}} D_{\text {gene }}
$$

where $k_{\text {gene }}$ is the maximum transcription rate of the gene, measured in Molar concentration per

minute; $k_{p o l}^{\max }$ is the maximum elongation speed (processivity rate) of the RNA polymerase, measured in number of bases synthesised per gene copy per cell per minute and neglecting pauses in polymerase activity; $L_{h n R N A}$ is the hnRNA transcript length, measured in number of bases per transcript (including introns); $N_{A}$ is Avogadro's constant; $V_{n u c}$ is the nuclear volume of a Zona Fasciculata (ZF) cell, measured in litres; and $D_{\text {gene }}$ is the gene dosage, or number of gene copies within each ZF cell.

The maximum processivity rate of the RNA Pol II in mammalian tissue culture cells is estimated at $71.6 \mathrm{nt} / \mathrm{sec}$ [Darzacq et al., 2007], corresponding to a maximum elongation speed of $k_{\text {pol }}^{\max } \approx$ $4.3 \mathrm{~kb} \mathrm{~min}^{-1}$. Regarding the gene dosage, we only have information for human Dax1 present as a duplicate, with both copies active. Thus, we will assume for all genes that $D_{\text {gene }}=2$. The only parameter that remains undetermined in Eq. 24 is the transcript length, which depends on the gene in question and we calculate below. 
For the Dax1 gene, which in rats is known to encode a hnRNA of $4129 \mathrm{~b}$ in length ${ }^{3}$, we estimate a maximum transcription rate of $k_{d a x 1}=1.33 \times 10^{-11} \mathrm{M} \mathrm{min}^{-1}$ (after splicing, the mature mRNA is reduced to $1.8 \mathrm{~kb}$ in length ${ }^{4}$ ). Similarly, the SF1 gene in rats is known to encode a hnRNA of $20825 b$ in length ${ }^{5}$, which we estimate will have a maximum transcription rate of $k_{s f 1}=2.64 \times$ $10^{-12} \mathrm{M} \mathrm{min}^{-1}$ (after splicing, the mature mRNA is barely $2182 b$ in length ${ }^{6}$ ). Lastly, the StAR gene is known to encode a hnRNA of $4643 b$ in length ${ }^{7}$, for which we estimate a maximum transcription rate of $k_{s t A R}=1.18 \times 10^{-11} \mathrm{M} \mathrm{min}^{-1}$ (after splicing, the predominant transcript variant is a $3.5 \mathrm{~kb}$ mRNA [Ariyoshi et al., 1998]). Thus:

$$
k_{\text {dax } 1}=1.33 \times 10^{-11} \mathrm{Mmin}^{-1}, \quad k_{s f 1}=2.64 \times 10^{-12} \mathrm{Mmin}^{-1}, \quad k_{s t A R}=1.18 \times 10^{-11} \mathrm{Mmin}^{-1} .
$$

which then implies the basal transcription rates are:

$$
\sigma_{\text {dax } 1}=6.65 \times 10^{-13} \mathrm{Mmin}^{-1}, \quad \sigma_{s f 1}=1.32 \times 10^{-13} \mathrm{Mmin}^{-1}, \quad \sigma_{s t A R}=5.91 \times 10^{-13} \mathrm{Mmin}^{-1} .
$$

The maturation rates of hnRNA into mRNA are likely subject to high variability given the postprocessing of transcripts depends on multiple, complex reaction steps (e.g. capping, polyadenylation, splicing, nuclear export) [Keene, 2007]. For the sake of simplicity, we'll assume their values are half the above maximum rates of gene expression. Thus, after halving their values and removing Molar units by multiplying by the product of Avogadro's constant and the nuclear volume, we arrive to:

$$
k_{\text {Dax } 1}=1.04 \mathrm{~min}^{-1}, \quad k_{S f 1}=0.21 \mathrm{~min}^{-1}, \quad k_{S t A R}=0.93 \mathrm{~min}^{-1} .
$$

We calculate the translation rate (protein synthesis) for any given transcript in a single ZF cell as:

$$
k_{\text {protein }}=\frac{k_{\text {rib }}}{L_{\text {poly }}}
$$

where $k_{\text {protein }}$ is the translation rate of the protein per minute per cell (keeping in mind this rate should be multiplied by the Molar concentration of mRNAs, so that protein concentration changes in units of $\left.M \min ^{-1}\right) ; k_{r i b}$ is the polypeptide elongation speed (processivity rate) of the ribosome, measured in number of codons translated (or aa's synthesised) per transcript per cell per minute and assuming ribosome stalling is already accounted for; and $L_{\text {poly }}$ is the polypeptide length, measured in number of aa's (we account for the polypeptide length instead of the mRNA length as UTR regions do not contribute to protein synthesis). We will use the average ribosome progression rate for translation in Mus musculus as a proxy for that of rat, which was unavailable. This is estimated at $5.6 \pm 0.5$ codons per second, which equals to $k_{\text {rib }} \approx 336 \mathrm{aa} \mathrm{min}^{-1}$ [Ingolia et al., 2011].

Considering the rat $D A X 1$ polypeptide is $472 a a$ in length ${ }^{8}$, we estimate a translation rate of $k_{D A X 1}=0.71 \mathrm{~min}^{-1}$. Similarly, the SF1 polypeptide in rats is $462 \mathrm{aa}$ in length ${ }^{9}$, which corresponds to a translation rate of $k_{S F 1}=0.73 \mathrm{~min}^{-1}$. Lastly, for the StAR protein precursor (the $37 k D a$ form StARp37), we can assume as a "rule of thumb" a molecular weight of 100-110 Da per average amino acid [Phillips et al., 2009]; assuming the newly synthesised StARp37 is $\sim 336$ aa in length (the 32 $\mathrm{kDa}$ form is $284 \mathrm{aa}$ long ${ }^{10}$ ), we can estimate a translation rate of $k_{S t A R p 37}=1 \mathrm{~min}^{-1}$. Thus:

$$
k_{D A X 1}=0.71 \mathrm{~min}^{-1}, \quad k_{S F 1}=0.73 \mathrm{~min}^{-1}, \quad k_{S t A R p 37}=1 \mathrm{~min}^{-1} .
$$

\footnotetext{
${ }^{3}$ http://www . ensembl.org/Rattus_norvegicus/Transcript/Exons?db=core; g=ENSRN0G00000003765; r=X : 54734385-54738513; $t=$ ENSRNOT00000005023

${ }^{4}$ http://www.ncbi.nlm.nih.gov/nuccore/NM_053317.1

${ }^{5}$ http://www.ensembl.org/Rattus_norvegicus/Transcript/Exons?db=core;g=ENSRN0G00000012682; $r=3$ : 22999616-23020441; t=ENSRNOT00000017651

${ }^{6}$ http://www.ncbi.nlm.nih.gov/nuccore/NM_001191099.1

7 http://www.ensembl.org/Rattus_norvegicus/Transcript/Exons?db=core; g=ENSRN0G00000015052; $r=16$ : 71036204-71040847; t=ENSRNOT00000020606

${ }^{8}$ http://www. uniprot.org/uniprot/P70503

${ }^{9}$ http://www. uniprot.org/uniprot/P50569

10 http: //www . uniprot.org/uniprot/P97826
} 
Lastly, we chose the parameters $k_{A C O R T}$ and $k_{G R}$ arbitrarily and allow them to vary during our model fitting to data as a means to probe the negative feedback strength. For these values, we assumed

$$
k_{A C O R T}=6.39 \times 10^{-12} \mathrm{Mmin}^{-1}, \quad k_{G R}=1 \times 10^{-12} \mathrm{Mmin}^{-1} .
$$

where the chosen value for $k_{A C O R T}$ is just 1 divided by the product of the nuclear volume and Avogadro's constant.

\subsection{Delays}

From the HPA model proposed in [Walker et al., 2010], the overall delay for ACTH signal transduction within the adrenal has been estimated to be $\tau_{\text {Adrenal }} \in[6.5,20]$ min. However, recent detailed experiments suggest two different time scales for the intra-adrenal network responsiveness, one for the slow genomic pathway and another for the fast, non-genomic one. Specifically, an intra venous (i.v.) pulse of ACTH produced rapid, transient increases in plasma CORT, with maximal responses achieved after 5 to 15 min (though some in vitro studies show ACTH can trigger CORT synthesis within $\sim 3 \mathrm{~min}$ ), and a decrease to almost basal levels at $\sim 30 \mathrm{~min}$. In contrast, StAR and P450scc hnRNA levels increased at $15 \mathrm{~min}$ following ACTH and decreased towards basal values after $30 \mathrm{~min}$ [Spiga et al., 2011a]. Hence, we can assume $\tau_{\text {Adrenal }}^{\text {Non-genomic }} \leq 5 \mathrm{~min}$ and $\tau_{\text {Adrenal }}^{\text {Genomic }} \approx 15 \mathrm{~min}$.

The case of the StAR gene is of particular interest since it is known to control the limiting step in CORT biosynthesis, which occurs very rapidly during the adrenal response to ACTH stimulation. From [Miller, 2013], we know that on a $15-60 \mathrm{~min}$ time scale, ACTH rapidly stimulates both the activation of pre-existing StAR protein and its de novo the synthesis. ACTH/cAMP doubles the activity of StAR proteins almost immediately [Arakane et al., 1997] and induces transcription of the StAR gene within minutes [Manna et al., 2009]. StARp37 then interacts with a complex macromolecular machine in the Outer Mitochondrial Membrane (OMM) that increases the flow of cholesterol from the OMM to the Inner Mitochondrial Membrane (IMM), where it becomes the substrate for CORT biosynthetic enzymes. Thus, we can assume that CORT synthesis is triggered almost instantaneously, whereas StAR gene expression kicks in until a few minutes later.

We can estimate the delays in the genomic pathway by taking into account the gene and hnRNA lengths, together with the processivity rate of the RNA Pol II and the ribosome. The StAR gene, for instance, is known to encode a hnRNA which is $4643 \mathrm{~b}$ long. As mentioned before, the maximum processivity rate of the RNA Pol II in mammalian tissue culture cells is estimated at $71.6 \mathrm{nt} / \mathrm{sec}=$ $4.3 \mathrm{~kb} / \mathrm{min}$ [Darzacq et al., 2007], which means the $S t A R$ hnRNA would take $\sim 1.08$ min to be transcribed. This transcriptional delay would be unusually short, and we should note that additional processes occur during transcript elongation, such as cumulative pauses of $\sim 4 \mathrm{~min}$ in average for polymerases on genes. After accounting for stalling effects on a variety of genes, the same study reports an average processivity rate for RNA Pol II as small as $6.3 \mathrm{nt} / \mathrm{sec}$. However, we must keep in mind that these measurements are subject to high variability, depending on the gene, the physiological conditions, and the cell type. For instance, in rat kidney cells the reactivation of serum responsive genes following serum deprivation suggests a synthetic rate of $1.1-1.4 \mathrm{~kb} / \mathrm{min}$ [Femino et al., 1998], whereas a more recent study performed on mouse ES cells determined that the RNA Pol II elongation rates ranged between 0.5 to $4 \mathrm{~kb} / \mathrm{min}$ [Jonkers et al., 2014]. In summary, several studies where measurements were taken by different techniques and in different conditions place the RNA Pol II elongation speed between 18 and $72 \mathrm{nt} / \mathrm{sec}$ [Swinburne and Silver, 2008], with most studies reporting values in the lower half of this range. Here, we will assume an average value of $30 \mathrm{nt} / \mathrm{sec}=1.8 \mathrm{~kb} / \mathrm{min}$.

Using this average processivity rate, the $s t A R$ transcriptional delay would be of $2.58 \mathrm{~min}$. Regarding transcript editing, we find the duration of an average splicing event has been estimated to last $\sim 30 \mathrm{sec}$ [Hnilicová and Staněk, 2011]. Considering the NCBI reports the StAR gene in Rattus norvegicus has 6 introns ${ }^{11}$, we can estimate it takes $\sim 3 \mathrm{~min}$ for st $A R$ hnRNA to mature into $S t A R$ mRNA, assuming introns are removed sequentially instead of simultaneously and neglecting transcript export to the translation site. Regarding translation, a ribosome progression rate of $5.6 \pm 0.5$

\footnotetext{
${ }^{11}$ http://www.ncbi.nlm.nih.gov/gene/25557
} 
codons per second in Mus musculus has been reported [Ingolia et al., 2011]. Considering a newly synthesised StARp37 precursor protein would have $\sim 336 a a$ in length (the $32 \mathrm{kDa}$ form is $284 a a$ long ${ }^{12}$ ), we can estimate a translation delay of 1 min assuming a "rule of thumb" of 100-110 Da per average amino acid [Phillips et al., 2009]. Following this, we estimated the delays for StAR gene expression as

$$
\tau_{S t A R}=5.58 \min , \quad \tau_{S t A R p 37}=1 \mathrm{~min} .
$$

Likewise, the SF1 gene in rats is known to encode a hnRNA which is $20825 b$ long. Using the same average RNA Pol II processivity rate as before, we estimate it would take $\sim 11.57 \mathrm{~min}$ to be transcribed. As the transcript has 6 introns in total ${ }^{13}$, we can estimate $\sim 3$ min for hnRNA to mature into mRNA, assuming introns are spliced sequentially and not simultaneously. Regarding translation, and considering the rat $S F 1$ polypeptide is $462 a a$ in length ${ }^{14}$, we can estimate a translation delay of $\sim 1.38 \mathrm{~min}$. Following this, we estimated the delays for the SF1 gene expression as

$$
\tau_{S f 1}=14.57 \min , \quad \tau_{S F 1}=1.38 \min .
$$

Lastly, the Dax1 gene in rats is known to encode a hnRNA which is $4129 \mathrm{~b}$ long. Using the same average RNA Pol II processivity rate as before, we estimate it would take $\sim 2.29$ min to be transcribed. As the transcript has only 1 intron in total ${ }^{15}$, we can estimate $\sim 0.5$ min for dax 1 hnRNA to mature into Dax $1 \mathrm{mRNA}$. Regarding translation, and considering the rat $D A X 1$ polypeptide is $472 \mathrm{aa}$ in length ${ }^{16}$, we can estimate a translation delay of $\sim 1.4 \mathrm{~min}$. Following this, we estimated the delays for Dax1 gene expression as

$$
\tau_{D a x 1}=2.79 \min , \quad \tau_{D A X 1}=1.4 \min .
$$

\subsection{Degradation Rates}

For simplicity, we'll assume that immature, uncapped, non-polyadenylated hnRNAs will have a degradation rate an order of magnitude faster than their mature mRNA counterparts. This results in half-lives for hnRNAs of $\sim 20 \mathrm{~min}$, comparable to those estimated previously for primary transcripts in a model of RNA metabolism in mammalian cells [Jackson et al., 2000]. However, after comparing the model predictions with our data, we required to slightly adjust some of our estimations. This likely arises from previously unaccounted context-dependent degradation processes, as we will see in what follows.

Dax 1 mRNA half-life is reported by [Ragazzon et al., 2006] to be $3 \mathrm{hrs}$. This value corresponds to a degradation rate of $\gamma_{\operatorname{Dax} 1}=3.85 \times 10^{-3} \mathrm{~min}^{-1}$. After fitting the model to data regarding the hnRNA half-life and considering a protein half-life of $6 \mathrm{hrs}$ [Osman et al., 2002], we arrive to the following estimates for the Dax1 gene:

$$
\gamma_{\text {dax } 1}=1.5 \times 10^{-2} \min ^{-1}, \quad \gamma_{\text {Dax } 1}=3.85 \times 10^{-3} \min ^{-1}, \quad \gamma_{D A X 1}=1.93 \times 10^{-3} \mathrm{~min}^{-1} .
$$

To the best of our knowledge, there is no data available regarding $S f 1 \mathrm{mRNA}$ stability. Nonetheless, we can assume that, because its mRNA is $\sim 400$ bases longer than the Dax 1 transcript, it degrades at a slower rate. Thus, after calculating a longer half-life in proportion to its length as compared to the Dax 1 transcript, we estimated a degradation rate for the $S f 1$ mRNA of $3.18 \times 10^{-3} \mathrm{~min}^{-1}$. This rate, however, had to be expressed in different units after the model fitting to data suggested that this degradation was better represented through a Michaelis-Menten mechanism. Thus, the $S f 1$ degradation rate was set to $\gamma_{S f 1}=2.03 \times 10^{-14} M_{m_{i n}}^{-1}$. The $s f 1$ hnRNA degradation rate, in contrast, was assumed an order of magnitude larger than its mRNA counterpart. In the proper units, this was set as $\gamma_{s f 1}=3.18 \times 10^{-2} \mathrm{~min}^{-1}$. Regarding the $S F 1$ protein, previously [Jacob et al., 2001] and [Chen et al., 2007] have estimated its half-life between 2.5 - 4hrs, corresponding to

\footnotetext{
12 http://www. uniprot.org/uniprot/P97826

13 http://www.ncbi.nlm.nih.gov/gene/83826

14 http://www . uniprot.org/uniprot/P50569

15 http://www.ncbi.nlm.nih.gov/gene/58850

16 http://www . uniprot.org/uniprot/P70503
} 
a degradation rate within the range $2.89 \times 10^{-3} \mathrm{~min}^{-1}$ to $4.62 \times 10^{-3} \mathrm{~min}^{-1}$. However, we must take into account the PKA-mediated stabilisation effects of ACTH upon SF1. For instance, [Æsøy et al., 2002] found a significant reduction in $S F 1$ protein amount in transfected COS-1 cells in the absence of PKA-C $\alpha$ overexpression measured $4 \mathrm{hrs}$ post chase (mean $\pm S E M=23.7 \pm 4.64 \%, P=0.0022$ ), compared with the amount of $S F 1$ protein after $1 \mathrm{hr}$, which was set to $100 \%$. In contrast, no significant reduction in $S F 1$ protein amount was observed after 4 hrs in cells cotransfected with PKA-C $\alpha$ ( $m e a n \pm S E M=77.2 \pm 9.5 \%, P=0.143$ ). Thus, it seems that the turnover of transiently expressed $S F 1$ was decreased by coexpression of PKA-C $\alpha$. Solving the differential equation for first order degradation, we find the degradation rate is given by:

$$
\gamma=\frac{1}{t} \ln \frac{x_{0}}{x(t)}
$$

which we can use to calculate $\gamma_{S F 1}^{P K A-}=8 \times 10^{-3} \mathrm{~min}^{-1}$ and $\gamma_{S F 1}^{P K A+}=1.44 \times 10^{-3} \mathrm{~min}^{-1}$. Here, we will use the first value, as by including the function $g_{S F 1}^{-}(A C T H)$ in the turnover term in Eq. 11 we account for the PKA-mediated stabilising effects of ACTH stimuli. Thus, for the SF1 gene, in the absence of ACTH stimulation, we will have:

$$
\gamma_{s f 1}=3.18 \times 10^{-2} \min ^{-1}, \quad \gamma_{S f 1}=2.03 \times 10^{-14} M_{m i n}^{-1}, \quad \gamma_{S F 1}=8 \times 10^{-3} \mathrm{~min}^{-1}
$$

The available information regarding $S t A R$ mRNA stability is a bit controversial, especially since two isoforms have been reported, each with different half-lives and the possibility of selective degradation in steroidogenic cells [Duan and Jefcoate, 2007]. As before, we could make an estimation based on its transcript length being twice as long as the Dax $1 \mathrm{mRNA}$. Following this, we estimated a degradation rate of $1.98 \times 10^{-3} \mathrm{~min}^{-1}$. However, at the moment of fitting the model to data from the ACTH i.v. pulse experiment, we found that this degradation rate had to be approximately twice as large. Accordingly, we have modified it to $\gamma_{S t A R}=3.96 \times 10^{-3} \mathrm{~min}^{-1}$. Likewise, the value of the st $A R$ hnRNA degradation was adjusted after fitting the model to data and was fixed at $\gamma_{s t A R}=1 \times 10^{-1} \mathrm{~min}^{-1}$. The increase in the $\gamma_{S t A R}$ degradation rate, when compared to the value we originally estimated for $S t A R$ mRNA is also supported by experimental evidence showing this gene contains three conserved AU-rich (AURE) element motifs known to mediate fast mRNA turnover [Zhao et al., 2005; Duan and Jefcoate, 2007]. Moreover, the measured half-life for the $3.5 \mathrm{~kb}$ $S t A R$ mRNA isoform corresponds to a degradation rate of $3.3 \times 10^{-3} \mathrm{~min}^{-1}$, which is close to our estimated value.

The estimation of the StARp37 protein turnover rate requires careful examination. According to [Arakane et al., 1997], the StAR protein has a short half-life, but a specific value is not reported. The half-life reported by [Ragazzon et al., 2006] is $\sim 5 \mathrm{hrs}$, with the primary source being [Granot et al., 2003]. However, this value refers to mitochondrial $30 k D a S t A R$, which lacks the N-terminus mitochondrial targeting sequence and it's not involved in cholesterol import into mitochondria. This sequence is cleaved upon the StAR $37 k D a$ import into mitochondria, a process that influences the rate of the StARp37 precursor cytosolic proteasome degradation, according to [Granot et al., 2003].

Both [Arakane et al., 1998] and [Granot et al., 2003] estimate that the StARp37 precursor has a half-life of $\sim 15 \mathrm{~min}$. However, this estimate accounts only for proteasome-assisted degradation, and the authors suggest that a fast import of the precursor into mitochondria would decrease the time it remains active. This would effectively increase the precursor's lability when both degradation and translocation processes are accounted for, thus decreasing the half-life to $\leq 5 \mathrm{~min}$ [Manna et al., 2009; Clark and Hudson, 2015]. This is consistent with previous estimations of StARp37 exhibiting a half-life of $5 \mathrm{~min}$ in mouse Y1 cells [Artemenko et al., 2001], and of $3-4 \mathrm{~min}$ in rat adrenal cells incubated at $37^{\circ} \mathrm{C}$ [Epstein and Orme-Johnson, 1991].

How then is it possible for adrenal cells to exhibit such a fast steroidogenic response while depending upon a precursor protein that is so labile? As it turns out, PKA-mediated ACTH stimulation induces rapid phosphorylation of the precursor StARp37, thus stabilising it in the cytosol and enhancing its activity. In other words, ACTH stimulation increases the residence time of StARp37 in the cytosol, thus increasing the rate of cholesterol import into mitochondria and enabling the fast response of the adrenal to synthesise CORT. 
In summary, we have that when mitochondrial import is blocked, the half-life of the StAR precursor is $15 \mathrm{~min}$, corresponding to $\gamma_{S t A R p 37}=4.62 \times 10^{-2} \mathrm{~min}^{-1}$; but when both mechanisms are blocked the precursor accumulates in the cytosol and its half-life increases up to $6.5 \mathrm{hrs}$, corresponding to a much lower turnover rate of $\mu_{\text {StARp } 37}=\gamma_{\text {StARp37 }}+\epsilon_{S t A R p 37}=1.78 \times 10^{-3} \mathrm{~min}^{-1}[\mathrm{Granot}$ et al., 2003]. In contrast, when both protein degradation and mitochondrial import are fully functional, and in the absence of ACTH stimulation, the protein is extremely labile, with a half-life of $\sim 3.5 \mathrm{~min}$, corresponding to $\mu_{\text {StARp } 37}^{A C T H}=\gamma_{\text {StARp } 37}+\epsilon_{\text {StARp } 37}=1.98 \times 10^{-1} \mathrm{~min}^{-1}$ [Epstein and Orme-Johnson, 1991]. However, upon PKA-mediated phosphorylation of the precursor after ACTH stimulation, the $S t A R p 37$ stabilises and its effective turnover rate drops by two orders of magnitude down to $\mu_{\text {StARp } 37}^{A C T H+}=\gamma_{\text {StARp } 37}+\epsilon_{\text {StARp } 37}=1.49 \times 10^{-3} \mathrm{~min}^{-1}$ [Clark and Hudson, 2015]. Thus

$$
\begin{aligned}
& \mu_{\text {StARp } 37}^{\text {ACTH }}=1.98 \times 10^{-1} \min ^{-1} \\
& \mu_{\text {StARp } 37}^{A C T H}=1.49 \times 10^{-3} \text { min }^{-1}
\end{aligned}
$$

The transition from a very high to a very low turnover rate for $S t A R p 37$ is represented in the second term in Eq. 18 by introducing the function $g_{S t A R p 37}^{-}(A C T H)$ (Eq. 19), which modulates the removal rate $\mu_{S t A R p 37}$. As the decrease in the turnover rate upon ACTH stimulation is quite large, we can fix the parameter as $\mu_{S t A R p 37}=1.98 \times 10^{-1} \mathrm{~min}^{-1}$ when no ACTH stimulation is present and let the function $g_{S t A R p 37}^{-}(A C T H)$ stabilise StARp37. Summarising for the StAR gene, the turnover rates are:

$$
\gamma_{s t A R}=1 \times 10^{-1} \mathrm{~min}^{-1}, \quad \gamma_{\text {StAR }}=3.96 \times 10^{-3} \mathrm{~min}^{-1}, \quad \gamma_{S t A R p 37}=1.98 \times 10^{-1} \mathrm{~min}^{-1} .
$$

Though the half-life of intra-adrenal corticosterone $(A C O R T)$ has not been measured directly, given its rapid export to the bloodstream we can assume it has a faster removal rate than its plasma counterpart. Assuming a half-life of $1 \mathrm{~min}$, we estimate a turnover rate of

$$
\mu_{A C O R T}=6.93 \times 10^{-1} \min ^{-1}
$$

Lastly, for the phosphorylated glucocorticoid receptor $(p G R)$, we find from [Bodwell et al., 1998] that the mouse GR is very stable, with a half-life of $\sim 18$ hrs (reduced down to $8-9$ hrs upon dexamethasone stimulation). However, we're interested in the half-life of the active, phosphorylated form of GR. This is likely to be much more short-lived, but nonetheless has not been measured. For the sake of simplicity, we assume it's half-life is the same as that of $A C O R T$, which implies a turnover rate of

$$
\gamma_{p G R}=6.93 \times 10^{-1} \min ^{-1}
$$

\subsection{Half-Maximum Constants}

The half-maximum constants (big $K$ 's) are arbitrary parameters that nonetheless capture the sensitivity of a process (e.g. synthesis or degradation) affecting the dynamics of a molecular species $Y$ as a function of the concentration of another species $X$. As the molecular mechanisms underlying these processes are often unknown, we model them as Michaelis-Menten and Hill type functions. In the latter case, when the Hill coefficient is high enough, these $K$ 's could also be interpreted as activation thresholds. Though arbitrary, their relative values are informative of how sensitive different nodes within the adrenal SRN are to common external stimuli (e.g. ACTH and cytokines).

Half-maximum constants were estimated manually after observing the system's time evolution and correcting our model for the timescales at which the peak of the transient response, together with the rising and decreasing phases, followed the experimental data. The estimated $K$ 's are listed in Table B and represented graphically in Fig. S9. The half-maximum constants that are neither dependent on ACTH nor cytokines, but are rather involved in Dax1, Sf1 and StAR mRNA degradation, in $A C O R T$ and $p G R$ synthesis, and in regulation of Dax1 and StAR gene promoters, were also fixed manually to values close to the range observed during their circadian dynamics.

Of particular interest are the constants $K_{\operatorname{Dax} 1}^{A C T H-l o}$ and $K_{\operatorname{Dax} 1}^{A C T H-h i}$ which, respectively, represent the lower and upper near half-maximum constants for a non-monotonous regulatory function (Eq. 5) 
that models dose-dependent effects of ACTH upon Dax1 mRNA stability (Fig. S4). We hypothesise that this dose-dependent degradation of $\operatorname{Dax} 1$ mRNA could be a post-transcriptional regulatory mechanism such as stress-induced non-sense mediated mRNA decay [He and Jacobson, 2015].

\begin{tabular}{|c|c|c|}
\hline \multicolumn{3}{|c|}{ Basal Transcription Rates } \\
\hline$\sigma_{d a x 1}=6.65 \times 10^{-13} M_{m_{i n}}^{-1}$ & $\sigma_{s f 1}=1.32 \times 10^{-13} \mathrm{Mmin}^{-1}$ & $\sigma_{s t A R}=5.91 \times 10^{-13} M_{m m i n^{-1}}$ \\
\hline \multicolumn{3}{|c|}{ Transcription, Translation and Activation Rates } \\
\hline$k_{\operatorname{dax} 1}=1.33 \times 10^{-11} \mathrm{M} \mathrm{min}^{-1}$ & $k_{s f 1}=2.64 \times 10^{-12} \mathrm{Mmin}^{-1}$ & $k_{s t A R}=1.18 \times 10^{-11} \mathrm{M} \mathrm{min}^{-1}$ \\
\hline$k_{\operatorname{Dax} 1}=1.04 \min ^{-1}$ & $k_{S f 1}=0.21 \mathrm{~min}^{-1}$ & $k_{S t A R}=0.93 \mathrm{~min}^{-1}$ \\
\hline$k_{D A X 1}=0.71 \mathrm{~min}^{-1}$ & $k_{S F 1}=0.73 \mathrm{~min}^{-1}$ & $k_{S t A R p 37}=1 \min ^{-1}$ \\
\hline$k_{A C O R T}=6.39 \times 10^{-12} M_{m_{i n}}^{-1}$ & $k_{G R}=1 \times 10^{-12} M_{\min ^{-1}}$ & \\
\hline \multicolumn{3}{|c|}{ Degradation Rates } \\
\hline$\gamma_{d a x 1}=1.5 \times 10^{-2} \mathrm{~min}^{-1}$ & $\gamma_{s f 1}=3.18 \times 10^{-2} \min ^{-1}$ & $\gamma_{s t A R}=1 \times 10^{-1} \mathrm{~min}^{-1}$ \\
\hline$\gamma_{\operatorname{Dax} 1}=3.85 \times 10^{-3} \min ^{-1}$ & $\gamma_{S f 1}=2.03 \times 10^{-14} \mathrm{Mmin}^{-1}$ & $\gamma_{S t A R}=3.96 \times 10^{-3} \min ^{-1}$ \\
\hline$\gamma_{D A X 1}=1.93 \times 10^{-3} \min ^{-1}$ & $\gamma_{S F 1}=8 \times 10^{-3} \min ^{-1}$ & $\mu_{S t A R p 37}=1.98 \times 10^{-1} \mathrm{~min}^{-1}$ \\
\hline$\mu_{A C O R T}=6.93 \times 10^{-1} \mathrm{~min}^{-1}$ & $\gamma_{p G R}=6.93 \times 10^{-1} \min ^{-1}$ & \\
\hline \multicolumn{3}{|c|}{ Delays } \\
\hline$\tau_{D a x 1}=2.79 \mathrm{~min}$ & $\tau_{S f 1}=14.57 \mathrm{~min}$ & $\tau_{S t A R}=5.58 \mathrm{~min}$ \\
\hline$\tau_{D A X 1}=1.4 \mathrm{~min}$ & $\tau_{S F 1}=1.38 \mathrm{~min}$ & $\tau_{\text {StARp } 37}=1 \mathrm{~min}$ \\
\hline \multicolumn{3}{|c|}{ "Half-Maximum Constants } \\
\hline $\begin{aligned} K_{\text {dax } 1}^{A C T H} & =90 \mathrm{pg} \mathrm{ml}^{-1} \\
K_{\text {Dax } 1}^{A C T H-l o} & =30 \mathrm{pg} \mathrm{ml}^{-1} \\
K_{\text {Dax } 1}^{A C T-h i} & =1000 \mathrm{pg} \mathrm{ml}^{-1} \\
K_{s f 1}^{A C T H} & =30 \mathrm{pg} \mathrm{ml}^{-1} \\
K_{S F 1}^{A C T H} & =70 \mathrm{pg} \mathrm{ml}^{-1} \\
K_{\text {stAR }}^{A C T H} & =70 \mathrm{pg} \mathrm{ml}^{-1} \\
K_{\text {StARp } 37}^{A C T H} & =70 \mathrm{pg} \mathrm{ml}^{-1}\end{aligned}$ & $\begin{aligned} K_{A C O R T} & =4.5 A U \\
K_{G} & =0.76 A U \\
K_{D} & =5.7 \times 10^{-5} M \\
K_{S f 1} & =1 \times 10^{-8} M \\
K_{S F 1} & =6.2 \times 10^{-7} M \\
K_{S t A R} & =1 \times 10^{-8} M \\
K_{S t A R p 37} & =4 \times 10^{-8} M\end{aligned}$ & $\begin{aligned} K_{d a x 1}^{I L 6}=K_{D a x 1}^{I L 6}=K_{D A X 1}^{I L 6} & =500 A U \\
K_{s f 1}^{I L 6} & =1000 A U \\
K_{S f 1}^{I L 6}=K_{S F 1}^{I L 6} & =100 A U \\
K_{S t A R}^{I L 6} & =2500 A U \\
K_{S t A R p 37}^{I L 6} & =500 A U \\
K_{s t A R}^{I L 1 \beta} & =200 A U \\
K_{s t A R}^{T N F \alpha}=K_{S t A R}^{T N F \alpha} & =60 A U \\
K_{p G R}^{T N F \alpha} & =20 A U\end{aligned}$ \\
\hline
\end{tabular}

Table B: Kinetic rates and other estimated parameter values. 


\section{Crosstalk Between the Adrenal SRN and the Immune Path- way}

Stimulation of rats with the bacterial lipopolysaccharide (LPS) endotoxin elicits a strong response not only at the HPA level but also on the immune system. In particular, LPS induces cytokines $T N F \alpha, I L 1 \beta$ and $I L 6^{17}$, which are known to act upon targets of the adrenal SRN and among themselves.

A previous mathematical model by [Malek et al., 2015] suggested a way in which LPS, TNF $\alpha$ and IL6 may interact with ACTH and CORT to mediate the adrenal response to inflammation. However, such a model assumed direct interactions of these cytokines upon ACTH, ignoring the steroidogenic pathway within adrenal cells. LPS effects on the immune system are more complex and start with the recruitment of macrophages to the adrenal cortex. This increases levels of $T N F \alpha$ in a first stage (peak at $60 \mathrm{~min}$ ), and of $I L 1 \beta$ and $I L 6$ (in T-lymphocytes) in a second stage (peaks at $120 \mathrm{~min}$ ) [Givalois et al., 1994]. While it is believed that the first stage is amplified through a positive feedback, in the second one $I L 1 \beta$ and $I L 6$ are known to inhibit rising levels of $T N F \alpha$. Moreover, though CORT is known to inhibit both $I L 1 \beta$ and $I L 6$, it has been proposed that $I L 1 \beta$ is involved in the initial activation of CORT production, whereas IL6 sustains it [Givalois et al., 1994; Kanczkowski et al., 2013]. These interactions between LPS and cytokines, together with the crosstalk between the immune pathway and the adrenal SRN (Table C), is summarised in Fig. S8.

Even though the interactions among cytokines are complex, we know that the output of the internal crosstalk within the immune pathway upon an LPS challenge delivers the measured levels of $T N F \alpha, I L 1 \beta$ and $I L 6$ (Fig. S7a-c). Thus, we can ignore the interactions within the immune pathway as long as we account for the effects of cytokines upon specific targets of the adrenal SRN. This effectively means that, during an LPS challenge, in addition to an ACTH input we will now use the time course of these cytokines as input functions. The task becomes complex as soon as we realise that the experimental evidence available comes from different cell types and animal models (Table C).

\subsection{Model Equations for the SRN with Cytokine Interactions}

We explored different network architectures and arrived to the scenario depicted in Fig. 5, which is associated to the following set of model equations, where the cytokine effects are shown in red:

$$
\begin{aligned}
& \dot{d a x} 1=\sigma_{d a x 1}+k_{d a x 1} f_{d a x 1}(S F 1, p G R) g_{\text {dax } 1}^{-}(A C T H) \phi_{d a x 1}(I L 6)-\gamma_{d a x 1} \operatorname{dax} 1, \\
& \dot{D a x} 1=k_{\text {Dax } 1} \operatorname{dax} 1_{\tau_{\text {Dax } 1}} \phi_{\text {Dax } 1}(I L 6)-\gamma_{\text {Dax } 1} \operatorname{Dax} 1 g_{\text {Dax } 1}^{\mp}(A C T H), \\
& D \dot{A X} 1=k_{D A X 1} \operatorname{Dax} 1_{\tau_{D A X 1}} \phi_{D A X 1}(I L 6)-\gamma_{D A X 1} D A X 1, \\
& \dot{s f} 1=\sigma_{s f 1}+k_{s f 1} g_{s f 1}^{+}(A C T H) \phi_{s f 1}(I L 6)-\gamma_{s f 1} s f 1, \\
& S \dot{f} 1=k_{S f 1} s f 1_{\tau_{S f 1}} \phi_{S f 1}(I L 6)-\gamma_{S f 1} h_{S f 1}(S f 1), \\
& S \dot{F} 1=k_{S F 1} S f 1_{\tau_{S F 1}} \phi_{S F 1}(I L 6)-\gamma_{S F 1} S F 1 g_{S F 1}^{-}(A C T H), \\
& s t \dot{A} R=\sigma_{s t A R}+k_{s t A R} f_{s t A R}(S F 1, D A X 1) g_{s t A R}^{+}(A C T H) \phi_{s t A R}(T N F \alpha) \phi_{s t A R}(I L 1 \beta)-\gamma_{s t A R} s t A R, \\
& S t \dot{A} R=k_{S t A R} s t A R_{\tau_{S t A R}} \phi_{S t A R}(T N F \alpha) \phi_{S t A R}(I L 6)-\gamma_{S t A R} h_{S t A R}(S t A R),
\end{aligned}
$$

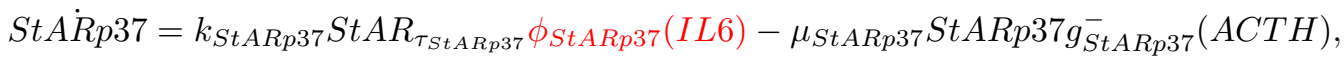

$$
\begin{aligned}
& A C \dot{O} R T=k_{A C O R T} f_{A C O R T}(\operatorname{StARp} 37)-\mu_{A C O R T} A C O R T, \\
& p \dot{G} R=k_{G R} f_{p G R}(A C O R T) \phi_{p G R}(T N F \alpha)-\gamma_{p G R} p G R .
\end{aligned}
$$

\footnotetext{
${ }^{17}$ The hyphen in $T N F-\alpha, I L-1 \beta$, and $I L-6$ labels was dropped to avoid confusion with a minus sign.
} 
where

$$
\begin{aligned}
& \phi_{\operatorname{dax} 1}(I L 6)=\frac{K_{\operatorname{dax} 1}^{I L 6}}{K_{d a x 1}^{I L 6}+I L 6}, \\
& \phi_{\operatorname{Dax} 1}(I L 6)=\frac{K_{\operatorname{Dax} 1}^{I L 6}}{K_{\operatorname{Dax} 1}^{I L 6}+I L 6}, \\
& \phi_{s t A R}(T N F \alpha)=1+\frac{T N F \alpha}{K_{s t A R}^{T N F \alpha}+T N F \alpha}, \\
& \phi_{D A X 1}(I L 6)=\frac{K_{D A X 1}^{I L 6}}{K_{D A X 1}^{I L 6}+I L 6}, \\
& \phi_{s t A R}(I L 1 \beta)=1+\frac{I L 1 \beta}{K_{\text {stAR }}^{I L 1 \beta}+I L 1 \beta}, \\
& \phi_{s f 1}(I L 6)=\frac{K_{s f 1}^{I L 6^{4}}}{K_{s f 1}^{I L 6^{4}}+I L 6^{4}}, \\
& \phi_{S t A R}(T N F \alpha)=1+\frac{T N F \alpha}{K_{S t A R}^{T N F \alpha}+T N F \alpha}, \\
& \phi_{S f 1}(I L 6)=\frac{K_{S f 1}^{I L 6^{4}}}{K_{S f 1}^{I L 6^{4}}+I L 6^{4}}, \\
& \phi_{S t A R}(I L 6)=\frac{K_{S t A R}^{I L 6}}{K_{S t A R}^{I L 6}+I L 6}, \\
& \phi_{S F 1}(I L 6)=\frac{K_{S F 1}^{I L 6}}{K_{S F 1}^{I L 6}+I L 6}, \\
& \begin{aligned}
\phi_{S t A R p 37}(I L 6) & =\frac{K_{S t A R p 37}^{I L 6}}{K_{S t A R p 37}^{I L 6}+I L 6} \\
\phi_{p G R}(T N F \alpha) & =\frac{K_{p G R}^{T N F \alpha}}{K_{p G R}^{T N F \alpha}+T N F \alpha}
\end{aligned}
\end{aligned}
$$

\begin{tabular}{|c|c|c|c|c|}
\hline Cytokine & Target & Sign & Cell type/species & Reference \\
\hline \multirow[t]{6}{*}{$T N F \alpha$} & StAR mRNA/protein & - & Rat testis Leydig tumor cells (LC-540) & [Sadasivam et al., 2015] \\
\hline & StAR mRNA/protein & - & Mouse macrophage cell line (RAW264.7) & [Ma et al., 2007] \\
\hline & DAX1 protein & + & Rat testis Leydig tumor cells (LC-540) & [Sadasivam et al., 2015] \\
\hline & StAR mRNA & + & Human adrenocortical cells (NCI-H295R) & [Tkachenko et al., 2011] \\
\hline & Cortisol & - & Bovine Zone Fasciculata cells & [Barney et al., 2000] \\
\hline & pGR & - & Human Airway Smooth Muscle cells & [Bouazza et al., 2012] \\
\hline$I L 1 \beta$ & StAR mRNA & + & Human adrenocortical cells (NCI-H295R) & [Tkachenko et al., 2011] \\
\hline \multirow[t]{8}{*}{$I L 6$} & StAR mRNA/protein & + & Bovine Zona Fasciculata cells & [McIlmoil et al., 2016] \\
\hline & Dax1 mRNA/protein & - & Bovine Zona Fasciculata cells & [McIlmoil et al., 2016] \\
\hline & SF1 mRNA/protein & + & Bovine Zona Fasciculata cells & [McIlmoil et al., 2016] \\
\hline & StAR mRNA/protein & - & Bovine Zona Reticularis cells & [McIlmoil et al., 2015] \\
\hline & Dax1 mRNA/protein & + & Bovine Zona Reticularis cells & [McIlmoil et al., 2015] \\
\hline & SF1 mRNA/protein & - & Bovine Zona Reticularis cells & [McIlmoil et al., 2015] \\
\hline & StAR mRNA & + & Human adrenocortical cells (NCI-H295R) & [Tkachenko et al., 2011] \\
\hline & Cortisol & + & Bovine Zona Fasciculata cells & [Barney et al., 2000] \\
\hline \multirow[t]{5}{*}{ LPS } & StAR $30 \mathrm{kDa}$ & - & Rat testis Leydig cells & $\begin{array}{c}\text { [Allen et al., 2004] } \\
\text { [Held Hales et al., 2000] }\end{array}$ \\
\hline & StAR $30 \mathrm{kDa}$ & + & Rat adrenal cells & [Held Hales et al., 2000] \\
\hline & StAR $37 \mathrm{kDa}$ & + & Rat testis Leydig tumor cells & [Allen et al., 2004] \\
\hline & StAR & + & Mouse Y1 cells & [Calejman et al., 2011] \\
\hline & Corticosterone & + & Rat adrenal cells & [Calejman et al., 2011] \\
\hline
\end{tabular}

As before, the half-maximum constants for the cytokine effects upon targets of the adrenal SRN can be understood as sensitivities. These were estimated by manually fitting the model predictions to experimental data from the LPS challenge experiments. The values are listed in Table B and represented graphically in Fig. S9d-f.

Table C: Cytokine effects upon targets within the adrenal SRN. 


\subsection{Scaling Model Outputs of Cytokine Targets}

Even though our model predicts the time evolution of hnRNA, mRNA, and protein concentrations, these predictions are inherently qualitative. This is mainly because we have approximated the mechanisms underlying gene regulation through Hill type functions and, to a lesser degree, because our model doesn't account for regulatory processes affecting mRNA and protein stability.

However, in order to make our model predictions comparable to the experimental data (expressed in $\mathrm{pg} / \mu \mathrm{g}$ for ACORT and as fold induction for all other variables), we have implemented a variable change to express the model predictions in terms of dimensionless arbitrary units. To do this consistently, all $x$ state variables in the model were transformed according to the formula:

$$
\hat{x}=\frac{x-x_{t=0}}{x_{\max }-x_{\min }} \sigma_{x}+\text { offset }
$$

where $x_{t=0}$ is the value of $x$ at the time the pulse is given, $x_{\max }$ and $x_{\min }$ are the maximum and minimum value of the response, and $\sigma_{x}$ is a scaling factor. The offset value was set to 1 for all state variables with data reported as fold induction, except for ACORT, which experiments reported as $\mathrm{pg} / \mu \mathrm{g}$ and for which we set an offset of 25 , following the baseline observed in ACTH i.v. pulse experiments.

The scaling factors were adjusted to reproduce the data of the ACTH i.v. pulse experiment, and these remained invariant when the model was used to predict the high s.c. ACTH pulse and LPS challenge data without cytokines (Table D). The only scenario where scaling factors were allowed to vary was after modifying the existing model to account for cytokine effects. Interestingly, in this latter case most scaling factors remain invariant, except for those multiplying variables targeted by cytokines. As before, this is because we have used Hill type functions to approximate the mechanisms by which these pro-inflammatory cytokines interact with targets of the SRN.

\begin{tabular}{|c|ccc|c|}
\hline Variable & $\begin{array}{c}\text { ACTH small } \\
\text { i.v. pulse }\end{array}$ & $\begin{array}{c}\text { ACTH high } \\
\text { s.c. pulse }\end{array}$ & $\begin{array}{c}\text { LPS challenge } \\
\text { without cytokines }\end{array}$ & $\begin{array}{c}\text { LPS challenge } \\
\text { with cytokines }\end{array}$ \\
\hline \hline ACORT & 300 & 300 & 300 & 300 \\
pGR & 3.5 & 3.5 & 3.5 & 0.4 \\
dax1 hnRNA & 0.9 & 0.9 & 0.9 & 0.9 \\
Dax1 mRNA & 0.4 & 0.4 & 0.4 & 0.8 \\
DAX1 protein & 0.1 & 0.1 & 0.1 & 0.6 \\
sf1 hnRNA & 0.6 & 0.6 & 0.6 & 0.6 \\
Sf1 mRNA & 0.5 & 0.5 & 0.5 & 0.1 \\
SF1 protein & 0.2 & 0.2 & 0.2 & 0.2 \\
stAR hnRNA & 3 & 3 & 3 & 3 \\
StAR mRNA & 2 & 2 & 2 & 2 \\
StARp37 protein & 0.1 & 0.1 & 0.1 & 2 \\
\hline
\end{tabular}

Table D: Scaling factors $\sigma_{x}$. Values in red correspond to non-statistically significant data. All scaling factors remain invariant in the model, except when the model is modified to account for cytokine inputs (last column) and only on molecular species targeted by cytokines (Fig. 5). 


\section{References}

Allen, J. A., Diemer, T., Janus, P., Hales, K. H., and Hales, D. B. (2004). Bacterial endotoxin lipopolysaccharide and reactive oxygen species inhibit leydig cell steroidogenesis via perturbation of mitochondria. Endocrine, 25(3):265-275.

Arakane, F., Kallen, C. B., Watari, H., Foster, J. A., Sepuri, N. B. V., Pain, D., Stayrook, S. E., Lewis, M., Gerton, G. L., and Strauss, J. F. (1998). The mechanism of action of steroidogenic acute regulatory protein (star) star acts on the outside of mitochondria to stimulate steroidogenesis. Journal of Biological Chemistry, 273(26):16339-16345.

Arakane, F., King, S. R., Du, Y., Kallen, C. B., Walsh, L. P., Watari, H., Stocco, D. M., and Strauss, J. F. (1997). Phosphorylation of steroidogenic acute regulatory protein (star) modulates its steroidogenic activity. Journal of Biological Chemistry, 272(51):32656-32662.

Ariyoshi, N., Kim, Y.-C., Artemenko, I., Bhattacharyya, K. K., and Jefcoate, C. R. (1998). Characterization of the rat star gene that encodes the predominant 3.5-kilobase pair mrna acth stimulation of adrenal steroids in vivo precedes elevation of star mrna and protein. Journal of Biological Chemistry, 273(13):7610-7619.

Artemenko, I. P., Zhao, D., Hales, D. B., Hales, K. H., and Jefcoate, C. R. (2001). Mitochondrial processing of newly synthesized steroidogenic acute regulatory protein (star), but not total star, mediates cholesterol transfer to cytochrome p450 side chain cleavage enzyme in adrenal cells. Journal of Biological Chemistry, 276(49):46583-46596.

Babu, P. S., Bavers, D. L., Beuschlein, F., Shah, S., Jeffs, B., Jameson, J. L., and Hammer, G. D. (2002). Interaction between dax-1 and steroidogenic factor-1 in vivo: increased adrenal responsiveness to acth in the absence of dax-1. Endocrinology, 143(2):665-673.

Barney, M., Call, G. B., McIlmoil, C. J., Husein, O. F., Adams, A., Balls, A. G., Oliveira, G. K., Miner, E. C., Richards, T. A., Crawford, B. K., et al. (2000). Stimulation by interleukin-6 and inhibition by tumor necrosis factor of cortisol release from bovine adrenal zona fasciculata cells through their receptors. Endocrine, 13(3):369-377.

Bodwell, J. E., Webster, J. C., Jewell, C. M., Cidlowski, J. A., Hu, J.-M., and Munck, A. (1998). Glucocorticoid receptor phosphorylation: overview, function and cell cycle-dependence. The Journal of steroid biochemistry and molecular biology, 65(1):91-99.

Bose, H. S., Lingappa, V. R., and Miller, W. L. (2002). Rapid regulation of steroidogenesis by mitochondrial protein import. Nature, 417(6884):87-91.

Bouazza, B., Krytska, K., Debba-Pavard, M., Amrani, Y., Honkanen, R. E., Tran, J., and Tliba, O. (2012). Cytokines alter glucocorticoid receptor phosphorylation in airway cells: role of phosphatases. American journal of respiratory cell and molecular biology, 47(4):464-473.

Calejman, C. M., Astort, F., Di Gruccio, J., Repetto, E., Mercau, M., Giordanino, E., Sanchez, R., Pignataro, O., Arias, P., and Cymeryng, C. (2011). Lipopolysaccharide stimulates adrenal steroidogenesis in rodent cells by a nf $\kappa$ b-dependent mechanism involving cox- 2 activation. Molecular and cellular endocrinology, 337(1):1-6.

Chen, W.-Y., Weng, J.-H., Huang, C.-C., and Chung, B.-c. (2007). Histone deacetylase inhibitors reduce steroidogenesis through scf-mediated ubiquitination and degradation of steroidogenic factor 1 (nr5a1). Molecular and cellular biology, 27(20):7284-7290.

Clark, B. J. and Hudson, E. A. (2015). Star protein stability in y1 and kin-8 mouse adrenocortical cells. Biology, 4(1):200-215.

Darzacq, X., Shav-Tal, Y., de Turris, V., Brody, Y., Shenoy, S. M., Phair, R. D., and Singer, R. H. (2007). In vivo dynamics of rna polymerase ii transcription. Nature structural molecular biology, 14(9):796-806. 
Duan, H. and Jefcoate, C. R. (2007). The predominant camp-stimulated $3.5 \mathrm{~kb}$ star mrna contains specific sequence elements in the extended 3 utr that confer high basal instability. Journal of molecular endocrinology, 38(1):159-179.

Epstein, L. and Orme-Johnson, N. (1991). Regulation of steroid hormone biosynthesis. identification of precursors of a phosphoprotein targeted to the mitochondrion in stimulated rat adrenal cortex cells. Journal of Biological Chemistry, 266(29):19739-19745.

Ermentrout, B. (2002). Simulating, analyzing, and animating dynamical systems: a guide to XPPAUT for researchers and students, volume 14. Siam.

Fan, W., Yanase, T., Wu, Y., Kawate, H., Saitoh, M., Oba, K., Nomura, M., Okabe, T., Goto, K., and Yanagisawa, J. (2004). Protein kinase a potentiates adrenal 4 binding protein/steroidogenic factor 1 transactivation by reintegrating the subcellular dynamic interactions of the nuclear receptor with its cofactors, general control nonderepressed-5/transformation/transcription domainassociated protein, and suppressor, dosage-sensitive sex reversal-1: a laser confocal imaging study in living kgn cells. Molecular Endocrinology, 18(1):127-141.

Femino, A. M., Fay, F. S., Fogarty, K., and Singer, R. H. (1998). Visualization of single rna transcripts in situ. Science, 280(5363):585-590.

Givalois, L., Dornand, J., Mekaouche, M., Solier, M., Bristow, A., Ixart, G., Siaud, P., Assenmacher, I., and Barbanel, G. (1994). Temporal cascade of plasma level surges in acth, corticosterone, and cytokines in endotoxin-challenged rats. American Journal of Physiology-Regulatory, Integrative and Comparative Physiology, 267(1):R164-R170.

Gorrigan, R. J., Guasti, L., King, P., Clark, A. J., and Chan, L. F. (2011). Localisation of the melanocortin-2-receptor and its accessory proteins in the developing and adult adrenal gland. Journal of molecular endocrinology, 46(3):227-232.

Granot, Z., Geiss-Friedlander, R., Melamed-Book, N., Eimerl, S., Timberg, R., Weiss, A. M., Hales, K. H., Hales, D. B., Stocco, D. M., and Orly, J. (2003). Proteolysis of normal and mutated steroidogenic acute regulatory proteins in the mitochondria: the fate of unwanted proteins. Molecular Endocrinology, 17(12):2461-2476.

Gummow, B. M., Scheys, J. O., Cancelli, V. R., and Hammer, G. D. (2006). Reciprocal regulation of a glucocorticoid receptor-steroidogenic factor-1 transcription complex on the dax-1 promoter by glucocorticoids and adrenocorticotropic hormone in the adrenal cortex. Mol Endocrinol, 20(11):2711-23.

He, F. and Jacobson, A. (2015). Nonsense-mediated mrna decay: degradation of defective transcripts is only part of the story. Annual review of genetics, 49:339-366.

Held Hales, K., Diemer, T., Ginde, S., Shankar, B. K., Roberts, M., Bosmann, H. B., and Hales, D. B. (2000). Diametric effects of bacterial endotoxin lipopolysaccharide on adrenal and leydig cell steroidogenic acute regulatory protein. Endocrinology, 141(11):4000-4012.

Hnilicová, J. and Staněk, D. (2011). Where splicing joins chromatin. Nucleus, 2(3):182-188.

Ingolia, N. T., Lareau, L. F., and Weissman, J. S. (2011). Ribosome profiling of mouse embryonic stem cells reveals the complexity and dynamics of mammalian proteomes. Cell, 147(4):789-802.

Iyer, A. K., Zhang, Y.-H., and McCabe, E. R. (2007). Lxxll motifs and af-2 domain mediate shp (nr0b2) homodimerization and dax1 (nr0b1)-dax1a heterodimerization. Molecular genetics and metabolism, 92(1):151-159.

Jackson, D. A., Pombo, A., and Iborra, F. (2000). The balance sheet for transcription: an analysis of nuclear rna metabolism in mammalian cells. The FASEB Journal, 14(2):242-254. 
Jacob, A. L., Lund, J., Martinez, P., and Hedin, L. (2001). Acetylation of steroidogenic factor 1 protein regulates its transcriptional activity and recruits the coactivator gen5. Journal of Biological Chemistry, 276(40):37659-37664.

Jasper, M. and Engeland, W. (1994). Splanchnic neural activity modulates ultradian and circadian rhythms in adrenocortical secretion in awake rats. Neuroendocrinology, 59(2):97-109.

Jonkers, I., Kwak, H., and Lis, J. T. (2014). Genome-wide dynamics of pol ii elongation and its interplay with promoter proximal pausing, chromatin, and exons. Elife, 3:e02407.

Kanczkowski, W., Chatzigeorgiou, A., Samus, M., Tran, N., Zacharowski, K., Chavakis, T., and Bornstein, S. R. (2013). Characterization of the lps-induced inflammation of the adrenal gland in mice. Molecular and cellular endocrinology, 371(1):228-235.

Keene, J. D. (2007). Rna regulons: coordination of post-transcriptional events. Nature Reviews Genetics, 8(7):533-543.

Kitay, J. I. (1961). Sex differences in adrenal cortical secretion in the rat 1. Endocrinology, 68(5):818824.

Kraemer, F. B. and Shen, W.-J. (2002). Hormone-sensitive lipase control of intracellular tri-(di-) acylglycerol and cholesteryl ester hydrolysis. Journal of lipid research, 43(10):1585-1594.

Ma, Y., Ren, S., Pandak, W., Li, X., Ning, Y., Lu, C., Zhao, F., and Yin, L. (2007). The effects of inflammatory cytokines on steroidogenic acute regulatory protein expression in macrophages. Inflammation research, 56(12):495-501.

Malek, H., Ebadzadeh, M. M., Safabakhsh, R., Razavi, A., and Zaringhalam, J. (2015). Dynamics of the hpa axis and inflammatory cytokines: Insights from mathematical modeling. Computers in biology and medicine, 67:1-12.

Manna, P. R., Cohen-Tannoudji, J., Counis, R., Garner, C. W., Huhtaniemi, I., Kraemer, F. B., and Stocco, D. M. (2013). Mechanisms of action of hormone-sensitive lipase in mouse leydig cells its role in the regulation of the steroidogenic acute regulatory protein. Journal of Biological Chemistry, 288(12):8505-8518.

Manna, P. R., Dyson, M. T., and Stocco, D. M. (2009). Regulation of the steroidogenic acute regulatory protein gene expression: present and future perspectives. Mol Hum Reprod, 15(6):32133 .

Manna, P. R., Wang, X.-J., and Stocco, D. M. (2003). Involvement of multiple transcription factors in the regulation of steroidogenic acute regulatory protein gene expression. Steroids, 68(14):11251134 .

McIlmoil, S., Call, G., Barney, M., Strickland, J., and Judd, A. (2015). Interleukin-6 inhibits adrenal androgen release from bovine adrenal zona reticularis cells by inhibiting the expression of steroidogenic proteins. Domestic animal endocrinology, 53:108-123.

McIlmoil, S., Strickland, J., and Judd, A. (2016). Interleukin 6 increases the in vitro expression of key proteins associated with steroidogenesis in the bovine adrenal zona fasciculata. Domestic animal endocrinology, 55:11-24.

Miller, W. L. (2013). Steroid hormone synthesis in mitochondria. Molecular and cellular endocrinology, $379(1): 62-73$.

Niakan, K. and McCabe, E. (2005). Dax1 origin, function, and novel role. Molecular genetics and metabolism, 86(1):70-83.

Nickerson, P. (1976). The adrenal cortex in spontaneously hypertensive rats. a quantitative ultrastructural study. The American journal of pathology, 84(3):545. 
Osman, H., Murigande, C., Nadakal, A., and Capponi, A. M. (2002). Repression of dax-1 and induction of sf-1 expression two mechanisms contributing to the activation of aldosterone biosynthesis in adrenal glomerulosa cells. Journal of Biological Chemistry, 277(43):41259-41267.

Phillips, R., Kondev, J., and Theriot, J. (2009). Physical biology of the cell; garland science: New york, 2009.

Ragazzon, B., Lefrançois-Martinez, A.-M., Val, P., Sahut-Barnola, I., Tournaire, C., Chambon, C., Gachancard-Bouya, J.-L., Begue, R.-J., Veyssière, G., and Martinez, A. (2006). Adrenocorticotropin-dependent changes in sf-1/dax-1 ratio influence steroidogenic genes expression in a novel model of glucocorticoid-producing adrenocortical cell lines derived from targeted tumorigenesis. Endocrinology, 147(4):1805-1818.

Rankin, J., Walker, J. J., Windle, R., Lightman, S. L., and Terry, J. R. (2012). Characterizing dynamic interactions between ultradian glucocorticoid rhythmicity and acute stress using the phase response curve.

Reul, J. and Kloet, E. D. (1985). Two receptor systems for corticosterone in rat brain: microdistribution and differential occupation. Endocrinology, 117(6):2505-2511.

Sadasivam, M., Ramatchandirin, B., Balakrishnan, S., and Prahalathan, C. (2015). Tnf- $\alpha$-mediated suppression of leydig cell steroidogenesis involves dax-1. Inflammation Research, 64(7):549-556.

Shen, W.-J., Patel, S., Natu, V., Hong, R., Wang, J., Azhar, S., and Kraemer, F. B. (2003). Interaction of hormone-sensitive lipase with steroidogeneic acute regulatory protein facilitation of cholesterol transfer in adrenal. Journal of Biological Chemistry, 278(44):43870-43876.

Song, K.-H., Park, Y.-Y., Park, K. C., Hong, C. Y., Park, J. H., Shong, M., Lee, K., and Choi, H.-S. (2004). The atypical orphan nuclear receptor dax-1 interacts with orphan nuclear receptor nur77 and represses its transactivation. Molecular Endocrinology, 18(8):1929-1940.

Spiga, F. and Lightman, S. L. (2015). Dynamics of adrenal glucocorticoid steroidogenesis in health and disease. Molecular and cellular endocrinology, 408:227-234.

Spiga, F., Liu, Y., Aguilera, G., and Lightman, S. L. (2011a). Temporal effect of adrenocorticotrophic hormone on adrenal glucocorticoid steroidogenesis: involvement of the transducer of regulated cyclic amp-response element-binding protein activity. J Neuroendocrinol, 23(2):136-42.

Spiga, F., Waite, E. J., Liu, Y., Kershaw, Y. M., Aguilera, G., and Lightman, S. L. (2011b). Acthdependent ultradian rhythm of corticosterone secretion. Endocrinology, 152(4):1448-57.

Spiga, F., Walker, J. J., Gupta, R., Terry, J. R., and Lightman, S. L. (2015). 60 years of neuroendocrinology: Glucocorticoid dynamics: insights from mathematical, experimental and clinical studies. Journal of Endocrinology, 226(2):T55-T66.

Spiga, F., Walker, J. J., Terry, J. R., and Lightman, S. L. (2014). Hpa axis-rhythms. Compr Physiol, $4(3): 1273-98$.

Sugawara, T., Kiriakidou, M., McAllister, J. M., Holt, J. A., Arakane, F., and Strauss, J. F. (1997a). Regulation of expression of the steroidogenic acute regulatory protein (star) gene: a central role for steroidogenic factor 1 . Steroids, 62(1):5-9.

Sugawara, T., Kiriakidou, M., McAllister, J. M., Kallen, C. B., and Strauss, J. F. (1997b). Multiple steroidogenic factor 1 binding elements in the human steroidogenic acute regulatory protein gene 5'-flanking region are required for maximal promoter activity and cyclic amp responsiveness. Biochemistry, 36(23):7249-7255.

Swinburne, I. A. and Silver, P. A. (2008). Intron delays and transcriptional timing during development. Developmental cell, 14(3):324-330. 
Tkachenko, I. V., Jääskeläinen, T., Jääskeläinen, J., Palvimo, J. J., and Voutilainen, R. (2011). Interleukins $1 \alpha$ and $1 \beta$ as regulators of steroidogenesis in human nci-h295r adrenocortical cells. Steroids, 76(10):1103-1115.

Veitia, R. A. (2002). Exploring the etiology of haploinsufficiency. Bioessays, 24(2):175-184.

Waite, E. J., McKenna, M., Kershaw, Y., Walker, J. J., Cho, K., Piggins, H. D., and Lightman, S. L. (2012). Ultradian corticosterone secretion is maintained in the absence of circadian cues. European Journal of Neuroscience, 36(8):3142-3150.

Walker, J. J., Terry, J. R., and Lightman, S. L. (2010). Origin of ultradian pulsatility in the hypothalamic-pituitary-adrenal axis. Proc Biol Sci, 277(1688):1627-33.

Watt, M. J., Holmes, A. G., Pinnamaneni, S. K., Garnham, A. P., Steinberg, G. R., Kemp, B. E., and Febbraio, M. A. (2006). Regulation of hsl serine phosphorylation in skeletal muscle and adipose tissue. American Journal of Physiology-Endocrinology and Metabolism, 290(3):E500-E508.

Windle, R., Wood, S., Shanks, N., Lightman, S., and Ingram, C. (1998). Ultradian rhythm of basal corticosterone release in the female rat: Dynamic interaction with the response to acute stress 1. Endocrinology, 139(2):443-450.

Winnay, J. N. and Hammer, G. D. (2006). Adrenocorticotropic hormone-mediated signaling cascades coordinate a cyclic pattern of steroidogenic factor 1-dependent transcriptional activation. Mol Endocrinol, 20(1):147-66.

Xu, B., Yang, W. H., Gerin, I., Hu, C. D., Hammer, G. D., and Koenig, R. J. (2009). Dax-1 and steroid receptor rna activator (sra) function as transcriptional coactivators for steroidogenic factor 1 in steroidogenesis. Mol Cell Biol, 29(7):1719-34.

Zhao, D., Duan, H., Kim, Y. C., and Jefcoate, C. R. (2005). Rodent star mrna is substantially regulated by control of mrna stability through sites in the 3'-untranslated region and through coupling to ongoing transcription. J Steroid Biochem Mol Biol, 96(2):155-73.

Æsøy, R., Mellgren, G., Morohashi, K.-I., and Lund, J. (2002). Activation of camp-dependent protein kinase increases the protein level of steroidogenic factor-1. Endocrinology, 143(1):295-303. 